Universidad Politécnica de Madrid

Escuela Técnica Superior de Ingenieros Navales

Departamento de Arquitectura, Construcción y Sistemas Oceánicos y Navales

\title{
Computational and experimental study of the influence of the free surface on rigid and deformable submerged structures
}

\author{
Héctor Rubén Díaz Ojeda
}

\author{
Ph.D. Thesis
}

Supervisor: Prof. Leo M. González

Co-Supervisor: Prof. Francisco J. Huera-Huarte

Doctorado en Ingeniería Naval y Oceánica 



\section{POLITÉCNICA}

Tribunal designado por la Comisión de Doctorado de la Universidad Politécnica de Madrid, en su reunión del día..... de .de $20 \ldots . .$.

Presidente:

Vocal:

Vocal:

Vocal:

Secretario:

Suplente:

Suplente:

Realizado el acto de defensa y lectura de la Tesis el día ..de. de $20 \ldots$ en la E.T.S.I. /Facultad.

Calificación

EL PRESIDENTE

LOS VOCALES 
I hated every minute of training, but I said, 'Don't quit. Suffer now and live the rest of your life as a champion'

Muhammad Ali 


\section{Abstract}

The aim of this thesis is to evaluate to what extent the immersion depth affects the hydrodynamics of a submerged body. Two different geometries have been selected.

Firstly, a deformable splitter plate is attached to the base of a stationary circular cylinder, and an uniform flow is imposed on the system, in the laminar regime. This structure is submerged at different depths and the dependence on the Cauchy, Reynolds and Froude number are studied. The deformation and the drag forces acting on the structure are analysed in detail in order to understand the effects that the free surface has on the problem. It was observed that the amplitude of the tip of the splitter plate grows as the Reynolds, Froude and Cauchy numbers are increased. However, as the depth grows, and the structure moves away from the interface, all measurements tend to saturate. For lower depths, the free surface works as a damper for the plate dynamics, reducing the oscillations. The physical mechanism of deformation based on the pressure difference at both sides of the plate is studied, monitoring how the pressure difference decreases as the structure approaches the free surface. The drag coefficient of the global structure (cylinder and plate) increases linearly with depth, but decreases when the viscosity of the fluid is reduced.

Secondly, the flow normal to a rectangular flat plate with sharp angles in the presence of the free surface and gravity has been studied numerically, using a 3D Large Eddy Simulation (LES) methodology. Previous numerical studies on this geometry consider either periodic assumptions or the laminar regime $R e \sim O\left(10^{3}\right)$. The numerical study described here, with a fully 3D simulation of the flat plate in the turbulent regime $R e \sim O\left(10^{5}\right)$ has not been reported before. Important differences have been found with respect to the laminar case or when periodic boundary conditions are assumed. The simulations are initially validated using a reference case of a plate with sharp corners and a single phase 3D-periodic configuration. The time averaged drag force, the velocity and pressure fields are compared against the case of the plate with smooth corners. Finally, in the case of the two-phase 3D numerical simulations, the drag force and the vorticity fields in the near wake of the plate are compared to recent experimental work of Satheesh and Huera-Huarte (2019). These experiments were used as a guideline for the computational set-up, consequently the value of most of the dimensionless parameters are the same. A second scenario where the free surface is replaced by a solid wall is also considered. Two important observations show up from this research, first: the characteristic unsteady frequencies associated to the two periodic regimes in the case involving smooth corners, is missing in this scenario. Second, a critical submergence depth (distance) between the upper part of the plate and the free surface (or solid wall) has been found, where the drag force shows a maximum value. These observations are discussed in 
relation to the pressure distribution, the vortex structures formed at the wake and the gap flow formed between the plate and the free surface. 


\section{Resumen}

El objetivo de esta tesis es el estudio y evaluación de la influencia de la superficie libre en estructuras sumergidas. Dos geometrías se han seleccionado.

Por un lado, una placa flexible empotrada en un cilindro rígido se ve afectada por un flujo en régimen laminar. La estructura se sumerge a diferentes profundidades y se estudia la dependencia de los números de Cauchy, Reynolds y Froude. La deformación de la placa y las fuerzas de drag actuando sobre la estructura son analizadas en detalle con la finalidad de entender los efectos que la superficie libre ocasiona en la estructura. Se observa que la amplitud de oscilación de la placa aumenta a medida que los números de Reynolds, Froude y Cauchy también aumentan. Sin embargo, cuando la profundidad crece y la estructura se aleja de la superficie libre, todos los resultados tienden a saturarse. Para profundidades pequeñas, la superficie libre actúa amortiguando la oscilación de la placa, reduciéndola. Se estudia la física de la deformación de la placa basada en la diferencia de presiones a ambas caras de la placa y se monitoriza cómo la diferencia de presión disminuye cuando la estructura se acerca a la superficie libre. El coeficiente de drag de la estructura formada por el cilindro más la placa aumenta linealmente con la profundidad, pero disminuye cuando la viscosidad del fluido es reducida.

Por otro lado, el flujo normal a una placa rectangular se estudia numéricamente usando la metodología Large Eddy Simulation (LES) teniendo en cuenta la superficie libre y la gravedad. Estudios numéricos previos en geometrías similares consideran periodicidad o flujo laminar. El estudio numérico, se basa en una simulación 3D con una placa rígida en régimen turbulento. Se encuentran importantes diferencias entre el caso laminar o asumiendo condiciones de contorno periódicas. Las simulaciones se validan inicialmente usando un caso de referencia con esquinas redondeadas y una configuración 3D periódica. La media aritmética de la fuerza de drag, la velocidad y los campos de presión se comparan en esta tesis con otras investigaciones a modo de validación. Finalmente, en el caso con dos fases y $3 D$, las fuerzas de drag y los campos de vorticidad cerca de la placa son comparados con el trabajo de Satheesh and Huera-Huarte (2019) Effect of the free surface on a at plate translating normal to the flow. Ocean Engineering 171, 458 - 468. Esos experimentos son usados para establecer el set-up en el numérico tomando en consideración los valores usados por el citado autor. Además se investigará el efecto del cambio de la superficie libre por una pared rígida. De estas investigaciónes se puede indicar, por un lado: las frecuencias inestables asociadas a los casos periódicos desaparecen cuando el mismo no es periódico. Otra conclusión es hay una profundidad crítica entre la parte superior de la placa y la superficie libre donde el drag muestra su máximo valor. Estas conclusiones son estudiadas teniendo en cuenta la distribución de presión, las estructuras de los vórtices formados y profundidad de la placa. 


\section{Acknowledgments}

Per saecula saeculorum I would always be extremely grateful to my family. The basis, the essence, the everything.

During my period as PhD student I have been working at these places: Buenos Aires and Santa Fé (Argentina), Dublin (Ireland), Tarragona and Madrid (Spain). It is impossible for me to thank individually all the people that have either been with me or influenced me during my research stay at these locations. Thanks to all of you.

I would also like to thank for the help, support, guidance, and teaching of my supervisors, Prof. Leo Miguel Gonzalez Gutierrez and Prof. Francisco Huera-Huarte. It was really nice to see how a problem can be approached from a numerical as well as an experimental point of view.

Most of the work was conducted at Madrid, working with Leo on numerical stuff and dealing with several research problems. The best part of working with him was all the criticisms and complaints about my job, and that probably got the best out of me. I think that it will be a very good learning for the rest of my life.

Though the research stay at Tarragona was short, working with Fran was certainly intense and focused on experiments. The main thing that I learnt from him was his practical way of doing things. He guided me but also gave me space in order to do things in my way, making mistakes and learning from them.

Thanks to Prof. Antonio Souto-Iglesias who gave me the opportunity of doing the PhD when I joined CEHINAV.

For the financial aid, thanks to all that have helped, especially the CEHINAV team.

Finally, I want to thank specially three guys:

Mr. Ernesto: He stayed with me through all times, both good and bad. What a life we had in Madrid!

Dr. Amadeo Morán: What to say about this guy... He made me cross the mainland in car (9 hours) for surfing in Portugal, he made me do $46 \mathrm{~km}$ trail marathon in Torcal ... Really thankful for the emotional help and the discussions of the paranormal activity of the numerical issues.

Dr. Sukruth Satheesh for the experimental help in our experiments in Tarragona. He became my friend, even though he almost killed me on an occasion with his spicy food. I also enjoyed the trips and walks around with him. 


\section{Contents}

1 Introduction 1

1.1 Fluid-Structure Interactions and Free Surfaces . . . . . . . . . . . . . . . . 2

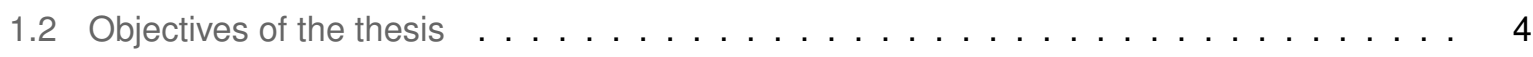

1.3 Layout of the thesis . . . . . . . . . . . . . . . . . . 5

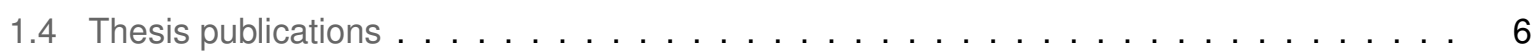

1.4 .1 Journal Papers . . . . . . . . . . . . . . . . . . . 6

1.4 .2 Conferences . . . . . . . . . . . . . . . . . . . 6

2 Literature review 9

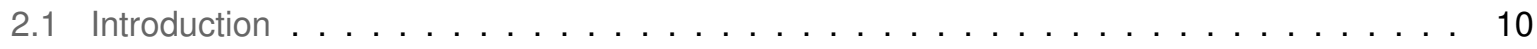

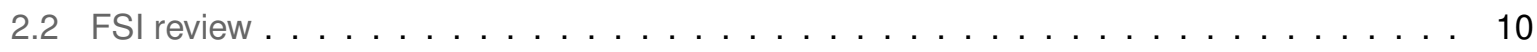

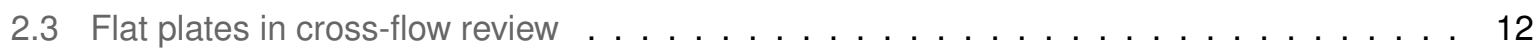

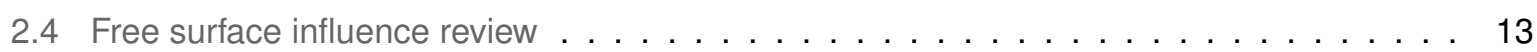

3 Physical and numerical modelling $\quad 15$

3.1 Introduction . . . . . . . . . . . . . . . . . . . . 16

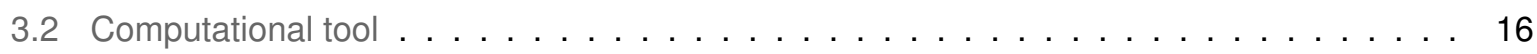

3.3 Fluid dynamic solver . . . . . . . . . . . . . . . . . . . . . . . . . . 17

3.3.1 Laminar assumption . . . . . . . . . . . . . . . . . . . 17

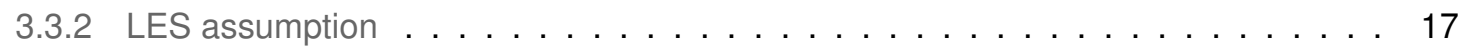

3.3.3 Volume of Fluid consideration . . . . . . . . . . . . . . . . . . . . . 19

3.4 Solid mechanics solver . . . . . . . . . . . . . . . . . . . . . . . . . . . . . . . . . 20

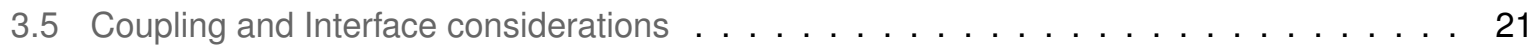

3.6 Dimensionless parameters . . . . . . . . . . . . . . . . . . . . . . 21

4 Laminar simulation of the effect of the free surface on a flexible splitter plate in the wake of a stationary cylinder 23

4.1 Introduction . . . . . . . . . . . . . . . . . . . . . . 24

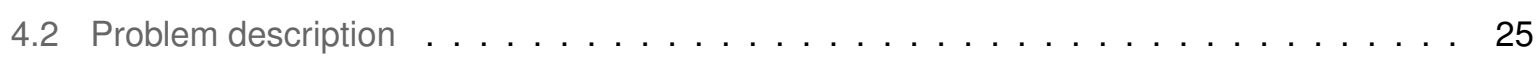

4.2 .1 Mesh description . . . . . . . . . . . . . . . . . . . . 25 


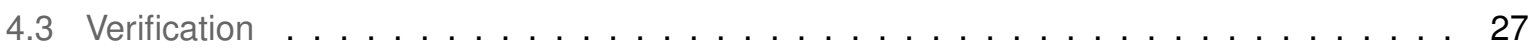

4.3.1 Main verification cases $(\mathrm{FSI} 2$ and $\mathrm{FSI}) \ldots \ldots \ldots \ldots \ldots$

4.3.2 Sensitivity analysis of the validation cases FSI2 and FSI3 . . . . . . . . . . 31

4.3.3 Splitter plate deformation in the presence of free surface . . . . . . . . . . 33

4.3.3.A Effect of Reynolds number . . . . . . . . . . . . . . . . . . . 34

4.3.3.B Effect of Cauchy number . . . . . . . . . . . . . . . . . . 39

4.3.3.C Effect of Froude number . . . . . . . . . . . . . . . . . 43

4.3.3.D Effect of Reynolds number . . . . . . . . . . . . . . . . . 44

4.3.3.E Effect of Cauchy number . . . . . . . . . . . . . . . . . 45

4.3.3.F Effect of Froude number . . . . . . . . . . . . . . . . . . 46

4.3.4 Transverse force frequency in the presence of the free surface $\ldots \ldots \ldots$. . . . 46

4.4 Chapter conclusions . . . . . . . . . . . . . . . . . . . . . . . . . . 50

5 Large Eddy Simulation of the flow past a normal thin plate with free surface 53

5.1 Introduction . . . . . . . . . . . . . . . . . . . . . . . . 54

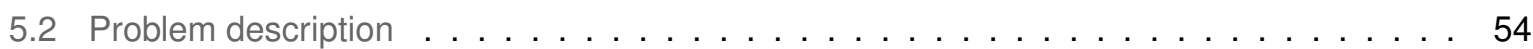

5.2 .1 Dimensionless parameters . . . . . . . . . . . . . . . . . . 55

5.3 Results: Validation of the numerical set-up . . . . . . . . . . . . . . 56

5.4 Results: Full 3D simulations involving free surface $\ldots \ldots \ldots \ldots$. . . . . . . . . 61

5.5 Chapter conclusions . . . . . . . . . . . . . . . . . . . . . . . . 75

6 Discussion and future work $\quad \mathbf{8 1}$

6.1 Chapter 4 considerations . . . . . . . . . . . . . . . . . . . . . 82

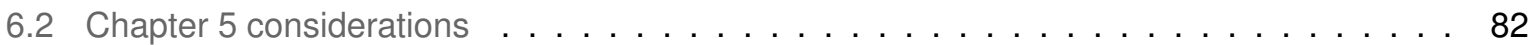

A Experimental study of the effect of free surface on forces generated by a cylinder with flexible splitter plate $\quad 91$

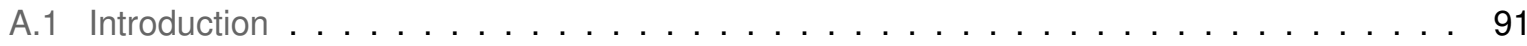

A.2 Experimental Setup . . . . . . . . . . . . . . . . . . . . . 92

A.3 Results . . . . . . . . . . . . . . . . . . . . . . . . . . 93

A.3.1 Variation of forces with depth $\ldots \ldots \ldots \ldots \ldots$. . . . . . . . . . . 94

A.3.2 Flexible splitter plate tip trajectory $\ldots \ldots \ldots \ldots \ldots \ldots \ldots$

A.3.3 Conclusions. . . . . . . . . . . . . . . . . . . . . . . . . . . 98 


\section{List of Figures}

1.1 Tacoma Narrows Bridge collapse. November 7, 1940 . . . . . . . . . . . . . 2

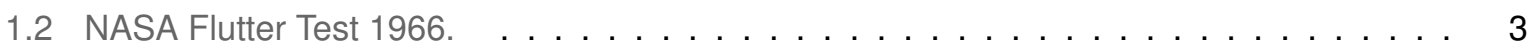

1.3 Left. Scheme of riser and riser with plate for vortex suppresition. Right. Tadpole example Fatorelli et al. (2018). . . . . . . . . . . . . . . . . . 4

1.4 Left. Swimmer legs in the butterfly style. Right. Whale flippers at different orientations

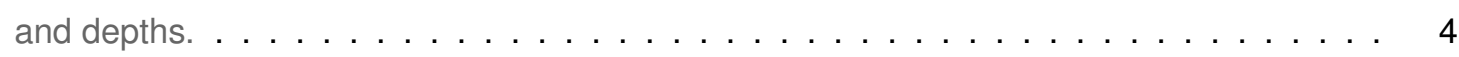

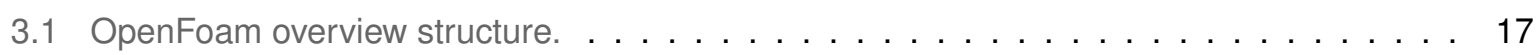

4.1 Problem set up and main geometric parameters . . . . . . . . . . . . . . 24

4.2 Schematic of the solid computational domain. . . . . . . . . . . . . . . 26

4.3 Structure and general view of the mesh 2 for the free surface case. The blue line indicates the initial free surface when two fluids are simulated. . . . . . . . . . . . . . . 27

4.4 Zoom of the mesh 2 over the cylinder and splitter plate. . . . . . . . . . . . . . 27

4.5 Horizontal and vertical time-varying displacements of point $A \ldots \ldots \ldots$

4.6 Instantaneous vorticity snapshots during a complete vortex shedding cycle of period $T$. Clockwise vorticity appears in blue (down to $\omega_{z_{\min }}^{*}=-8$ ) and counter-clockwise in red (up to $\omega_{z_{\max }}^{*}=8$ ). Vorticity is presented in dimensionless form, defined as $\omega_{z}^{*}=\frac{\omega_{z} D}{U_{\infty}} \ldots 30$

4.7 Superimposed view of the deflections of the splitter plate $\ldots \ldots \ldots \ldots$

4.8 Comparison of the amplitude displacement (left) and drag force coefficient (right) for some the additional cases. Case 0: Turek case FSI 3. Case 1: Turek case FSI 3, but using uniform inflow profile $U=2$. Case 2: Case 1 with a rigid splitter plate. Case 3: Turek case FSI 3, but using a rigid splitter plate. Case 4: The top wall is set at $10.1 D$ from the cylinder center, and the bottom wall is set at $6 D$ from the cylinder center. Uniform inflow profile $U=2$. Case 5: The top wall is set at $10.1 D$ from the cylinder center, and the bottom wall is set at $2 D$ from the cylinder center. Uniform inflow profile $U / \bar{U}=1 \ldots \ldots \ldots \ldots$. . . 
4.9 Evolution of vertical displacement of the tip of the plate corresponding with Reynolds number $R e=200$, Froude number $F r=2$, Cauchy number $C y=7.14 e-4$ and $h / D=0.45 .33$

4.10 Vertical amplitudes of the plate tip versus distance to the bottom wall $a / D$. The Froude $F r=2$ and the Cauchy numbers $C y=7.14 \cdot 10^{-4}$ are kept fixed. . . . . . . . . .

4.11 Vertical amplitudes of the plate tip versus depth for different Reynolds number values and different $h / D$ depths. The Froude $F r=2$ and the Cauchy numbers $C y=7.14 \cdot 10^{-4}$ are kept fixed. Case 4, with uniform inflow velocity $U / \bar{U}=1$ and FSI3 properties (Table 4.3), has been included for comparison in a dashed blue line. . . . . . . . . . . . .

4.12 Snapshot of the normalized pressure field contour plot at $h / D=1.6$ during a complete shedding cycle of period $T$. The rest of parameters are: $\mathrm{Fr}=2, C y=7.14 \cdot 10^{-4}$ and

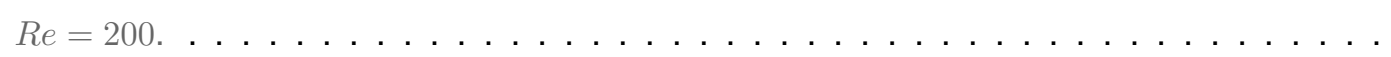

4.13 Snapshot of the normalized pressure field contour plot at $h / D=0.8$ during a complete shedding cycle of period $T$. The rest of parameters are: $\mathrm{Fr}=2, C y=7.14 \cdot 10^{-4}$ and

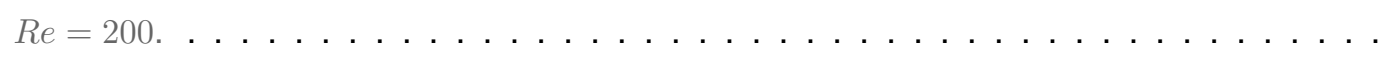

4.14 Pressure field difference along the plate for three different depths, when the tip of the plate is at the highest $(A)$ and lowest $(B)$ positions. The Froude $(F r=2)$ and the Cauchy numbers $(\mathrm{Cy}=7.14 \mathrm{e}-4)$ are kept fixed. . . . . . . . . . . . . . . . . . .

4.15 Snapshot of the vorticity field contour plot at $h / D=0.45$ during a complete shedding cycle of period $T$. Clockwise vorticity appears in blue (down to $\omega_{z_{\min }}^{*}=-8$ ) and counterclockwise in red (up to $\omega_{z_{\text {max }}}^{*}=8$ ). The rest of parameters are: $F r=2, C y=7.14 \cdot 10^{-4}$

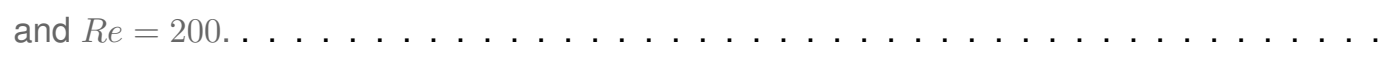

4.16 Snapshot of the vorticity field contour plot at $h / D=1.60$ during a complete shedding cycle of period $T$. Clockwise vorticity appears in blue (down to $\omega_{z_{m i n}}^{*}=-8$ ) and counterclockwise in red (up to $\omega_{z_{\max }}^{*}=8$ ). The rest of parameters are: $\mathrm{Fr}=2, \mathrm{Cy}=7.14 \cdot 10^{-4}$

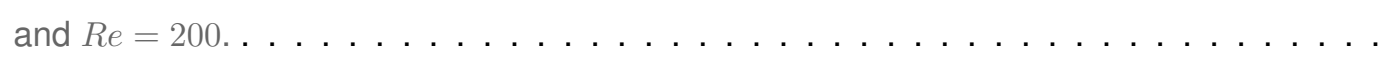

4.17 Vertical periodic displacement signal of point $A$ for $R e=200$ and $h / D=0.45$. Different positions $P_{i} \quad i=1, \ldots 9$ at different instants during one period have been selected for analysis. . . . . . . . . . . . . . . . . . .

4.18 Contour normalized pressure field snapshots $\ldots \ldots \ldots \ldots \ldots \ldots$

4.19 Normalized pressure values along the top, bottom of the structure and normalized pressure difference at the snapshot $\ldots \ldots \ldots \ldots \ldots \ldots \ldots$

4.20 Splitter plate oscillation versus depth for different Cauchy numbers. The Froude and Reynolds numbers are constant with values: $F r=2$ and $R e=200$. Case 4, with uniform inflow velocity $U / \bar{U}=1$ and FSI3 properties (Table 4.3), has been included for comparison in a dashed blue line. . . . . . . . . . . . . . . . . . . . . 
4.21 Comparison between the numerical simulations by changing the Cauchy number and keeping the Froude $F r=2$ and the Reynolds $R e=200$ numbers constant. . . . . . .

4.22 Vertical oscillation amplitude of point $A$ by changing the Froude number and keeping constant the Cauchy number $C y=7.14 \cdot 10^{-4}$ and the Reynolds number $R e=200$. Case 4, with uniform inflow velocity $U / \bar{U}=1$ and FSI3 properties (Table 4.3), has been included for comparison in a dashed blue line. . . . . . . . . . . . . . . . . . . . . .

4.23 Drag coefficient versus depth for different Reynolds numbers while keeping constant the Froude $F r=2$ and Cauchy numbers $C y=7.14 \cdot 10^{-4}$. Case 4 , with uniform inflow velocity $U / \bar{U}=1$ and FSI3 properties (Table 4.3), has been included for comparison in a dashed blue line. . . . . . . . . . . . . . . . . . . . . . . . . . . . . . .

4.24 Averaged drag coefficients $C_{D}$ changing the Cauchy number and keeping constant the Froude $F r=2$ and the Reynolds $R e=200$ numbers. Case 4, with uniform inflow velocity $U / \bar{U}=1$ and FSI3 properties (Table 4.3), has been included for comparison in a dashed blue line. . . . . . . . . . . . . . . . . . . . . . . . . . . .

4.25 Averaged drag coefficient versus depth for different Froude numbers, while keeping constant the Cauchy $C y=7.14 \cdot 10^{-4}$ and the Reynolds $R e=200$ numbers. Case 4, with uniform inflow velocity $U / \bar{U}=1$ and FSI3 properties (Table 4.3), has been included for comparison in a dashed blue line.

4.26 Frequencies versus depth for different Reynolds numbers, while keeping constant the Froude $\mathrm{Fr}=2$ and the Cauchy $C y=7.14 \cdot 10^{-4}$ numbers. . . . . . . . . . . . .

4.27 Frequencies versus depth for different Cauchy numbers, while keeping constant the Froude

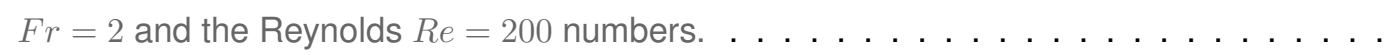

4.28 Frequencies versus depth for different Froude numbers, while keeping constant the Cauchy $C y=7.14 \cdot 10^{-4}$ and the Reynolds $R e=200$ numbers. . . . . . . . . . . . .

5.1 Schematic of the computational domain. The air phase is only present in the case that involves free surface simulations, not in the case with the rigid wall . . . . . . . . . 54

5.2 Scheme of the computational domain for the plane $z=0 \ldots \ldots \ldots \ldots \ldots$

5.3 Spectrum of the resolved streamwise velocity fluctuations obtained at the point $x / b=1$, $y / b=0.5$ and $-5 / 3$ slope $\ldots \ldots \ldots \ldots \ldots \ldots \ldots \ldots$

5.4 Evolution of the drag(left) and lift(right) coefficients for a single fluid in the 3D-periodic case. 59

5.5 Power spectra of the time evolution of the drag(left) and lift(right) coefficients for the single fluid 3D-periodic case. . . . . . . . . . . . . . . . . . . . . . . 60

5.6 Time and spanwise averaged streamwise velocity $u$ along the wake centerline $y=0 \ldots \quad 60$

5.7 Time evolution of velocities: $\bar{u}($ top $), \bar{v}$ (middle), $\bar{w}$ (bottom) along the line $x / b=1, y / b=0$ for

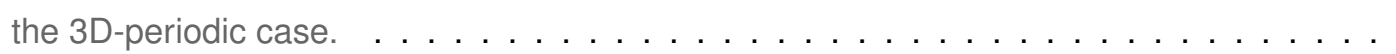


5.8 Time and spanwise mean pressure coefficient $\left\langle C_{p}>\right.$ distribution on the front and back sides of the plate for the 3D-periodic case. . . . . . . . . . . . . . . . .

5.9 Averaged drag coefficients $C_{D}$ for different depths when the plate aspect ratio $A R=1$ and $R e=30 \times 10^{3}$ (top), $R e=45 \times 10^{3}$ (middle), $R e=60 \times 10^{3}$ (bottom). For $R e=60 \times 10^{3}$ the results for the complementary problem using a top wall instead of free surface have been added for comparison. . . . . . . . . . . . . . . . . . . .

5.10 Averaged drag coefficient $C_{D}$ against depth $d / b$ when the plate aspect ratio $A R=0.5$ and $R e=30 \times 10^{3}$ (top), $R e=45 \times 10^{3}$ (middle), $R e=60 \times 10^{3}$ (bottom)

5.11 Time and cross averaged dimensionless pressure coefficient $\left\langle C_{p}>\right.$ difference between front and back sides along $y / b$ for $A R=1$ and $R e=60 k$ for different depths $d / b$. . . . . .

5.12 Integral value of the pressure coefficient difference between both sides of the plate along the vertical direction. . . . . . . . . . . . . . . . . . . . . .

5.13 Time and cross averaged dimensionless pressure coefficient $<C_{p}>$ along $y / c$ for $A R=1$ and $R e=60 k$ for different wall distances $d / b$. Circles represent the front side, $-v$ letter when $d / b$ case is indicated, while asterisks represent the back side, $a$ letter when $d / b$ case is indicated. Right: Time and cross averaged dimensionless pressure coefficient $<C_{p}>$ difference between front and back sides along $y / b$ for $A R=1$ and $R e=60 k$ for different depths $d / b . \ldots \ldots \ldots \ldots \ldots \ldots \ldots \ldots \ldots \ldots \ldots \ldots$

5.14 Spectra of the resolved cross-stream velocity fluctuations obtained at the mid $-\operatorname{span} x / b=$ 1 and $z / b=0$ for three different depths; $d / b=0, d / b=0.125$ and $d / b=0.625$ when $A R=1$ and $R e=60 k \ldots \ldots \ldots \ldots \ldots \ldots \ldots \ldots \ldots \ldots \ldots \ldots \ldots \ldots \ldots \ldots \ldots \ldots$

5.15 Evolution of the drag(left) and lift(right) coefficients when $A R=1, R e=60 \mathrm{k}$ and $d / b=1.25$

5.16 Power spectra of the time evolution of the drag(left) and lift(right) coefficients when $A R=$ $1, R e=60 k$ and $d / b=1.25 \ldots \ldots \ldots \ldots \ldots \ldots \ldots$

5.17 Streamlines of the time and spanwise averaged flow field in the near wake at different depths $d / b . \ldots \ldots \ldots \ldots \ldots \ldots \ldots \ldots \ldots \ldots \ldots \ldots$

5.18 Streamlines of the time and spanwise averaged flow field in the near wake at different wall

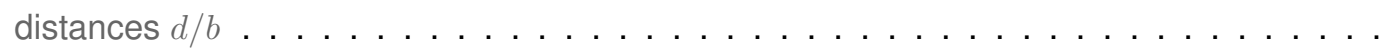

5.19 Comparison between the computed dimensionless vorticity $\omega_{Z}$ versus PIV experiments in Satheesh and Huera-Huarte (2019). The color scale is $\omega_{Z} \in[-16,16]$ for both results. Snapshot of the vorticity contour at when $R e=45 k$ and $A R=0.5$. . . . . . . . . . .

5.20 Snapshot of the dimensionless pressure field $p^{*}$ (left) and streamlines at $R e=60 k, A R=$

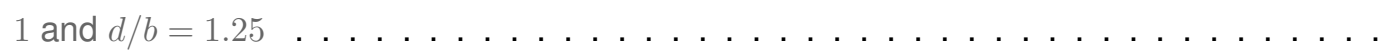

5.21 Isosurface plot of $Q=1.0$ in the wake of normal flat plate at $R e=60 \mathrm{k}, \mathrm{d} / \mathrm{b}=1.25$ (deepest case) using a solid wall at the top boundary. . . . . . . . . . . . . . . . . . . . . . 
5.22 Isosurface plot of $Q=1.0$ in the wake of normal flat plate at $R e=60 k, d / b=1.25$ (deepest case) with free surface. . . . . . . . . . . . . . . . . . . . . . . 77

5.23 Isosurface plot of $Q=1.0$ in the wake of normal flat plate at $R e=60 k, d / b=0$ using a solid wall at the top boundary. . . . . . . . . . . . . . . . . . . . . . .

$5.24 \mathrm{Top}$ (top) and lateral(bottom) views of the isosurface plot of $Q=1.0$ in the wake of normal flat plate at $R e=60 k, d / b=0$ with free surface . . . . . . . . . . . . . 79

A.1 Schematic of a cylinder-flexible splitter plate model . . . . . . . . . . . . . 93

A.2 Schematic representation of the experimental setup in the water tunnel. Cylinder axis is perpendicular to the viewing plane. . . . . . . . . . . . . . . . . . . . 94

A.3 Variation of drag coefficient with submergence depth ratio . . . . . . . . . . . . 95

A.4 Variation of lift coefficient with submergence depth ratio . . . . . . . . . . . . . 96

A.5 Tip motion characteristics of a flexible splitter plate with submergence depth ratio . . . . 97 


\section{List of Tables}

4.1 Number of cells of each subdomain, fluid and structural and cell size close to the beam $\left(A_{h} / D^{2}\right)$. The total number of cells (structure+fluid) in Turek and Hron (2007) mesh is also included for reference. . . . . . . . . . . . . . . . . . . . . 26

4.2 Comparison between different meshes and different time steps with the benchmark case 26

4.3 Parameters used in the $\mathrm{FSI} 2$ and $\mathrm{FSI} 3$ validation cases $\ldots \ldots \ldots \ldots$

4.4 Comparison between the amplitudes and frequencies of the horizontal and vertical displacements of point $A$ (splitter plate tip), obtained by Turek et al. (2010) and Pisacreta (2017), for case FSI3.

4.5 Comparison between of the amplitudes and frequencies of the horizontal and vertical displacements of extreme plate point $A$ and the Hron-Turek results Turek et al. (2010) for case FSI2. . . . . . . . . . . . . . . . . . . . . . . . . . . . . 30

4.6 Natural frequencies numbers computed analytically for the different Cauchy numbers. . . 48

5.1 Summary of the different parameters of the geometries proposed by Tian et al. (2014) and Satheesh and Huera-Huarte (2019) and the ones used in this work, see figure 5.1. For the case proposed in Satheesh and Huera-Huarte (2019), $G / b$, and $J / b$ are nondimensionalized with the height of the plate $b=0.16 m \ldots \ldots \ldots \ldots$. . . . . . 56

5.2 3D-periodic LES set-up validation. . . . . . . . . . . . . . . . . . . . . . 57

A.1 Splitter plate geometry . . . . . . . . . . . . . . . . . . . . . . 92 


\section{Acronyms}

AR Aspect Ratio, ratio of square of the span to the exposed area

$k_{b}$ Bending stiffness parameter

$C y$ Cauchy number

$C_{D}$ Dimensionless drag coefficient

$C_{L}$ Dimensionless lift coefficient

DNS Direct Numerical Simulation

Fr Froude number

$D_{h}$ Hydraulic diameter

LES Large Eddy Simulation

$M$ Mass ratio

E Young's modulus

Re Reynolds number

I Sectional moment of inertia 


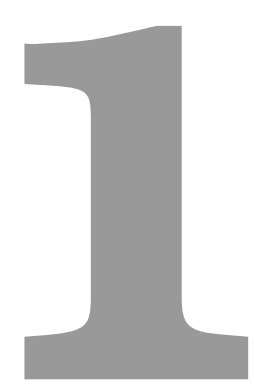

\section{Introduction}

\section{Contents}

1.1 Fluid-Structure Interactions and Free Surfaces $\ldots \ldots \ldots \ldots \ldots$

1.2 Objectives of the thesis . . . . . . . . . . . $4 \ldots \ldots$

1.3 Layout of the thesis $\ldots \ldots \ldots \ldots \ldots \ldots \ldots \ldots \ldots \ldots$

1.4 Thesis publications $\ldots \ldots \ldots \ldots \ldots \ldots \ldots \ldots \ldots \ldots \ldots \ldots \ldots$ 


\subsection{Fluid-Structure Interactions and Free Surfaces}

It has been almost eighty years since the Tacoma Narrows Bridge collapsed on the morning of the 7th November 1940, see figure 1.1. The presence of high winds buffeted the bridge structure and consequently it swayed considerably. Even though the bridge towers were made of strong structural carbon steel, the bridge was being tossed back and forth wildly for an approximately $64 \mathrm{~km} / \mathrm{s}$ wind speed producing the bridge collapse. This incident was a large scale empirical paradigmatic event for science and engineering since this problem might be considered as the elementary example of the resonance phenomenon. In this engineering problem, the winds produced aeroelastic flutter in a structure, the bridge, matching the natural frequency of the structure with the excitation frequency produced by the fluid and consequently breaking it. From a global point of view, in this problem two systems are involved, fluid and structure: this illustrates the importance of studying the coupling between them.

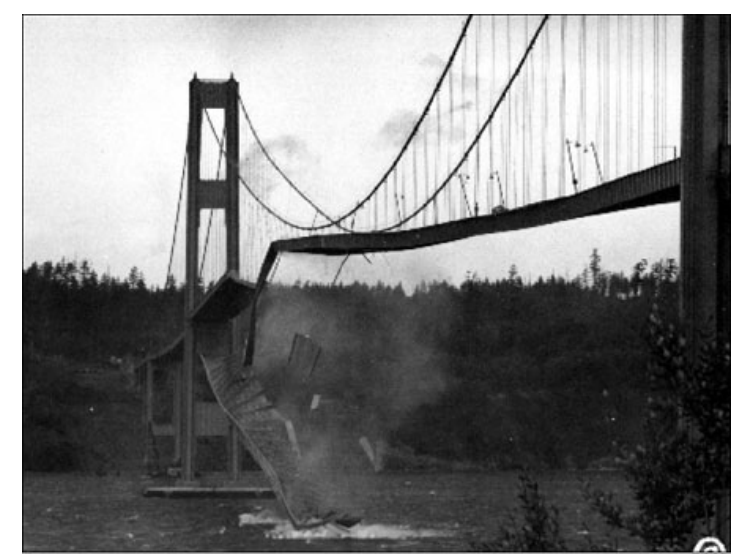

Figure 1.1: Tacoma Narrows Bridge collapse. November 7, 1940.

In 1966, the National Aeronautics and Space Administration (NASA) in one of their tests, see figure 1.2, encountered the phenomenon of flutter in flexible structures. The structures were affected by the presence of a fluid showing the importance of considering not only the fluid or solid separately when a new design is studied, but also the coupling between them. This new idea come out since the flow induces motion and deformation in the structure and the motion of that structure introduces changes in the flow which makes changes in the initial structural problem.

Those two examples might be considered as representative problems where the structure and fluid are involved together in a coupled system that cannot be treated separately. Fluid-Structure Interaction $(\mathrm{FSI})$ are complex problems to solve, that demand the coupling of several simpler systems in a global system, where the output of the first sub-system is the input of the second and vice-versa. Such complexity implies that most of the research conducted until now is limited to the study of just one subsystem, for instance the fluid dynamics around a non-deformable structure or the structural dynamics 


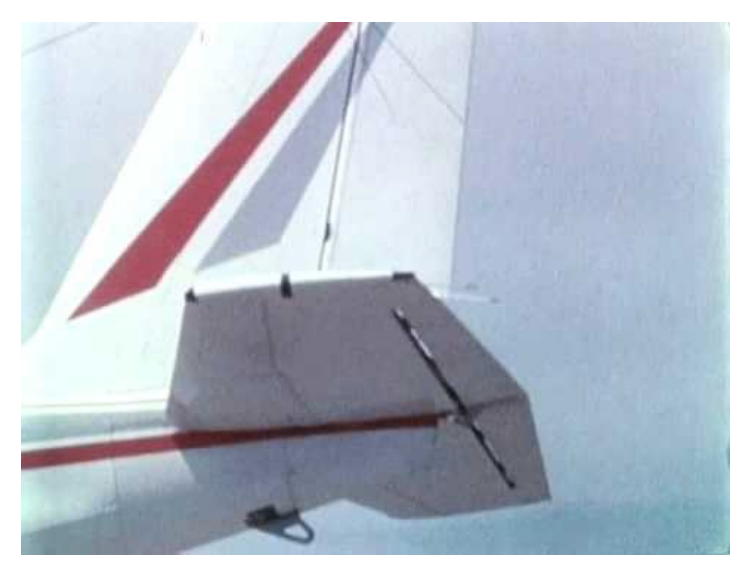

Figure 1.2: NASA Flutter Test 1966.

of a problem with fixed loads. Nowadays, most of the predictions and studies in FSI are provided by experimental studies due to the computational cost of the numerical simulations. Nonetheless, this is changing due to the development of the computational resources which allow to parallelize these complex problems, consequently being able to complete the numerical predictions of different FSI problems.

In the marine field, where most of the unexplored resources are partially or completely submerged in water, consequently affected by strong currents and waves, the study of flexible elements such as risers or mooring lines becomes important and challenging problems, see figure 1.3. Even in the most simple cases such as bio-propulsion systems, the presence of FSI problems, is quite frequent. For instance, the propulsion system of a swimming tadpole was studied in Liu et al. (1996), see figure 1.3. This study was conducted in order to check whether a 2D CFD simulation was able to match the thrust, power and other derived data when compared to experimental data. This kind of research allows to perform deeper studies about this kind of biological problems in the future.

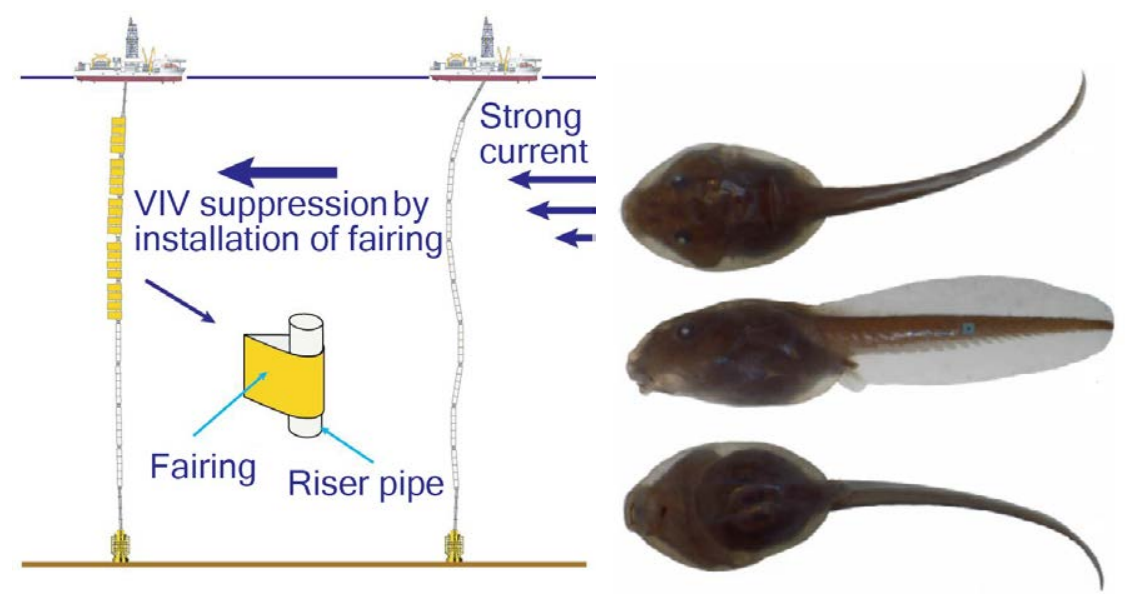

Figure 1.3: Left. Scheme of riser and riser with plate for vortex suppresition. Right. Tadpole example Fatorelli et al. (2018). 
These studies, experimental and/or numerical, imply that the structure is subject to flow-induced forces depending on the pressure and velocity flow fields. The flow is completely determined by the position of the boundaries and the fluid distribution when more than one fluid is involved in the computation. In those examples where two fluids are simulated numerically, the free surface deformation is determined by the passive scalar that characterizes the fluid phase that is transported by the fluid velocity field.

In several FSI problems the free surface presence alters the behavior of the structures involved and its dynamical response is interesting from different points of view. For instance, most swimmers want to maximize the self-propulsion obtained from their legs, see figure 1.4, the angular acceleration of the legs and the interaction with the free surface determine the efficiency of the swimmer. A similar example is represented by the flippers on humpback whales, see figure 1.4. The self-propulsion of the whale body is affected by FSI but also by the interaction between the two fluids, air and water, involved in the problem. The power demanded for propulsion and/or orientation of the whale body depends among other factors on the depth and position of the flipper.
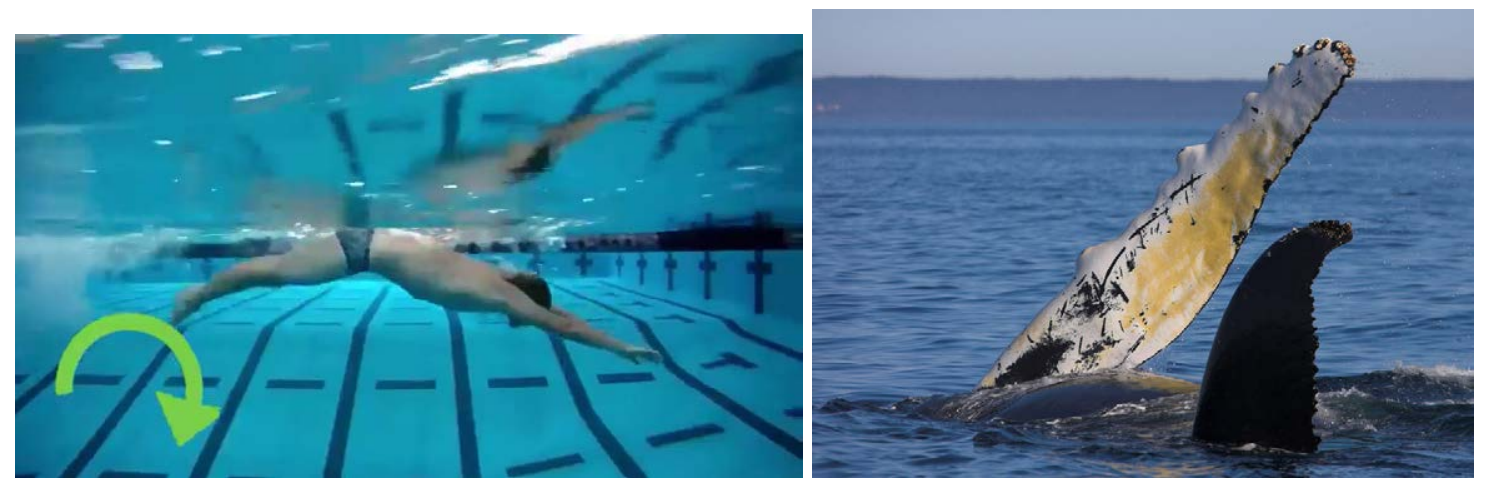

Figure 1.4: Left. Swimmer legs in the butterfly style. Right. Whale flippers at different orientations and depths.

\subsection{Objectives of the thesis}

This thesis is focused on understanding the physics involved when a rigid or deformable bluff body is immersed in a fluid flow. This work studies the fluid dynamics effects on a 3D submerged rigid plate at high Reynolds numbers in the presence of the free surface. The work progresses changing the rigid bluff body by a deformable structure with elastic properties. Not only the behaviour of the forces and fluid dynamics when the body is placed close to the free surface are studied, but also their influence on the structure. Since very few previous studies are conducted taking into account the free surface influence, this work focuses on the numerical understanding of the influence of the flow pass a flexible splitter plate attached to a cylinder in laminar 2D.

Moreover, an experimental campaign is carried out to reveal the dynamics of the splitter plate and 
the fluid forces acting on the cylinder.

\subsection{Layout of the thesis}

The present thesis document includes the results of two different numerical studies.

Chapter 2 presents a summary of the state of the art that has been considered during the development of this thesis.

Chapter 3 presents the governing equations and a complete description of the numerical methodology used in this work. Equations for the fluid, solid, and free surface, the numerical tool OpenFOAM, the way to deal with the coupled system and other details of the numerical technique are also included.

Chapter 4 studies a deformable splitter plate attached to the aft of a stationary circular cylinder in the laminar regime. A validation of the numerical techniques is performed by reproducing the results previously obtained by other authors in a problem considered as a classical benchmark. After this initial validation with a single fluid, the gravitational force and a second fluid were included in the simulation, where the deformation of the free surface is also studied. The structure is placed at different depths and the dependence on the three main non-dimensional numbers, such as the Cauchy, Reynolds and Froude number are studied. The deformation and the drag forces acting on the structure are analyzed in detail in order to understand the effects that the free surface has on the problem.

Chapter 5 introduces the problem of a rigid flat plate placed normal to incoming flow. Two validations are performed: first, a numerical validation using the numerical work presented in Tian et al. (2012) and a second one against the experimental results obtained for different depths in Satheesh and HueraHuarte (2019). Finally, the validation methodology and the new result contributed by the numerical results are evaluated. The dependance on the shape of the corner plate, the wake vorticity evolution and the analysis of the forces on the plate are also compared with other similar geometries such as the ones used by other referenced authors. The numerical results obtained are oriented to complement the experimental work performed in Satheesh and Huera-Huarte (2019). An important goal is to prove that the LES numerical methodology selected for this case is able to deal with this kind of simplified geometries even when a free surface is involved, which in the future will open the door to more complex problems.

The final chapter covers a discussion from the different problems, limitations and possible future tasks on the topic studied. 


\subsection{Thesis publications}

\subsubsection{Journal Papers}

H.R. Díaz-Ojeda, F.J. Huera-Huarte, L.M.González. Large-eddy simulation on the influence of the free surface on a Rigid Stationary Rectangular cylinder. Under review in Physics of Fluids

Sukruth Satheesh, H.R. Díaz-Ojeda, L.M.González. F.J. Huera-Huarte. Effect of free surface on forces generated by a cylinder with flexible splitter plate. Under review in Journal of Offshore Mechanics and Arctic Engineering

Jerónimo Domingo, Luis Pérez-Rojas, H.R. Díaz-Ojeda. Numerical investigation of vortex-induced vibration of a vertical riser in cross flow at high Reynolds Under review in Journal of Marine Science and Technology

H.R. Díaz-Ojeda, L.M.González, F.J. Huera-Huarte. On the influence of the free surface on a stationary circular cylinder with a flexible splitter plate in laminar regime. Journal of Fluids and Structures Vol. 87, Pages 102-123, May 2019

Amadeo Moran-Guerrero, L.M.González, Adriana Oliva-Remola, H.R. Díaz-Ojeda. On the influence of transition modeling and crossflow effects on open water propeller simulations. Ocean Engineering, Vol. 156, 101 - 119. 2018;

L.M.González, E. Ferrer, H.R. Díaz-Ojeda. Onset of three-dimensional flow instabilities in lid-driven circular cavities. Physics of Fluids 29, 064102 - 2017;

\subsubsection{Conferences}

Roberto Sosa, Alejandro D. Otero, H.R. Díaz-Ojeda, L.M.González. Vortex Induced Vibrations of an elastically mounted cylinder arranged in Side by Side configuration with a stationary cylinder. ENIEF 2019 , XIV Congreso sobre Métodos Numéricos y sus Aplicaciones, 5 al 7 de Noviembre de 2019, Santa Fe, Argentina

F.J. Huera-Huarte, Sukruth Satheesh, H.R. Díaz-Ojeda, L.M.González. Flow features of rigid plates towed in cross-flow near the free-surface. Workshop on Fluid Mechanics, Granada, July 22 - 23, 2019

H.R. Díaz-Ojeda, L.M.González, F.J. Huera-Huarte. Evaluation of the impact of a free surface in a typical FSI problem.. ASME 2018 37th Int. Conf. on Ocean, Offshore and Arctic Engineering. OMAE2018 - June 17-22, 2018, Madrid, Spain.

Adriana Oliva-Remola, Luis Pérez-Rojas,H.R. Díaz-Ojeda Ship Roll Damping Estimation: A Comparative Study of Different Roll Decay Tests Proceedings of the 13th International Conference on the Stability of Ships and Ocean Vehicles, 16-21 September 2018, Kobe, Japan.

V.R. Bernal-Colio, J.L. Cercos-Pita, Calderon-Sanchez, H.R. Díaz-Ojeda, Ricardo Abad, . Souto- 
Iglesias Numerical modeling of the forced motion dynamics of antiroll tank with OpenFOAM. ASME 2018 37th Int. Conf. on Ocean, Offshore and Arctic Engineering. OMAE2018 - June 17-22, 2018, Madrid, Spain.

Juan Manzanero, Gonzalo Rubio, Esteban Ferrer, H.R. Díaz-Ojeda, and Eusebio Valero Dispersiondiffusion analysis for advection problems with non-constant coefficients: Applications to discontinuous Galerkin formulations. 19h International Conference on Finite Elements in Flow Problems - FEM 2017. 5-7 April. Rome, Italy. 
CHAPTER 1. INTRODUCTION 


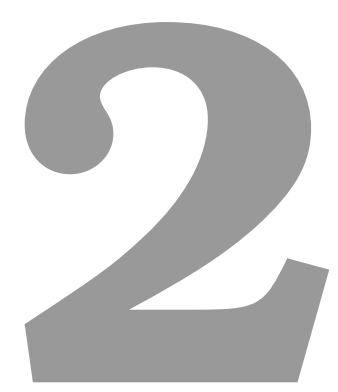

\section{Literature review}

\section{Contents}

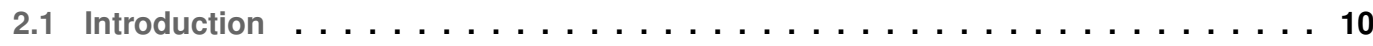

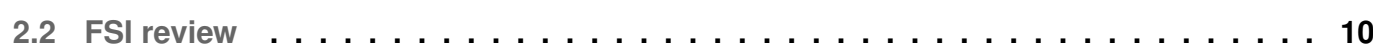

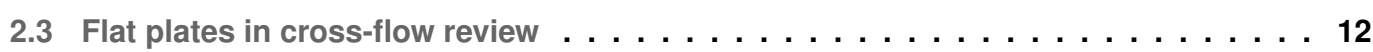

2.4 Free surface influence review $\ldots \ldots \ldots \ldots \ldots \ldots \ldots \ldots \ldots$ 


\subsection{Introduction}

Canonical bluff bodies such as circular or rectangular cylinders and the analysis of Fluid-Structure Interactions (FSI) with large-scale flow-induced structural deformation have important implications and significant relevance in engineering problems. They are frequently found for instance in energy-harvesting devices that extract energy from the ocean waves and sea currents, ocean structures, bridges, offshore rigs, complex biomedical flow etc. In this chapter a review of some of the most relevant previous work taken into account is presented. A FSI bibliography study is done in 2.2 being followed by rigid flat plates study in 2.3. Finally, the free surface influence review is presented in 2.4 .

\subsection{FSI review}

A deeper knowledge about FSI problems where submerged objects are involved is very demanded due to the fact that future energy projects point towards to the sea as one of the most important energy resources for future applications. A wide variety of energy-harvesting devices are now being developed, to extract energy from ocean waves and sea currents. In the ocean environment, systems are forced to interact with the sea dynamics and consequently are deformed by sea loads. Unexpected failures can have catastrophic economical implications since offshore costs tend to be very large. Generally, submerged elastic structures are being constantly deformed by the sea currents near water's surface. When the motion of such submerged objects is equal to one of the natural frequencies, the fatigue life decreases rapidly. Nowadays, empirical methods are the leading approach for simulating these phenomena, but numerical methods based on $\mathrm{FSI}$ are becoming more and more attractive as computational capabilities are constantly improving. Researchers have recently focused on mechanisms such as the use of a flexible splitter plate not only for modulation of vortex shedding or flow-induced vibration suppression Assi et al. (2009); Huera-Huarte (2014), but also for energy harvesting. Taylor et al. (2001); Allen and Smits (2001) examined the feasibility of a flexible splitter plate, which is made of a piezoelectric membrane attached to a bluff body for electricity generation from vortex-shedding-induced vibration of the plate.

From the research perspective, the interaction of shear flows with the ocean's surface open the doors to challenging fluid mechanics problems. The flow around submerged objects imply fully three dimensional complex vortical structures which interact with the free surface. The vortex shedding behind a bluff body causes unsteady pressure drops on the near surface of the body leading to drag and lift fluctuations. The vibration magnitude of a splitter plate attached on a bluff body in its wake region, can be characterized by the unsteady pressure loading due to vortex shedding, and by the flexibility of the plate. Due to its practical importance in many engineering problems, much attention has been devoted to 
manipulating vortex shedding with active and/or passive means. Among them, attaching a splitter plate to the cylinder base has been known to be one of the most successful ways to control vortex shedding Lou et al. (2016). As the shape of both submerged objects and the free surface deform in time, the flow field around the object also changes. In those situations where the deformations of the object are large and strongly affect the flow field, the fluid and the solid equations are fully coupled, and consequently a two-way FSI must be implemented.

As numerical methods play an important role in predicting hydrodynamic motion and forces on these submerged objects, an FSI benchmark for flow-induced deformation of thin elastic structures is necessary. The study of the self-sustained oscillation of an elastic splitter plate attached to the lee side of a rigid cylinder in two-dimensional laminar channel flows Turek et al. (2010) is currently considered a standard benchmark for FSI. Previous studies documented the effects of the material properties of the structure and flow conditions on the response of the structure. In the absence of free surface, the behaviour of the plate is a function of the vortex-shedding frequency and the natural frequency of the plate. The vortex shedding frequency is determined by the shape of the body and the Reynolds number, while the natural frequency of the plate is determined by Young's modulus and density and its geometric parameters, especially the length of the plate. Despite increasing engineering interest, Lee and You (2013) stated that the quantitative relationship between the characteristics of the vortices shed, the material and geometric properties of the plate that describe the influence on the drag and lift acting on the bluff body and the vibration of the plate, are not well understood even without free surface. In Bhardwaj and Mittal (2012) the effect of Reynolds number, material properties and geometric non-linearity on the plate displacement as well as its frequency in the FSI benchmark proposed by Turek et al. (2010) was quantified. They showed that the oscillation frequency of the plate varies linearly with dilatational wave speed inside the plate (or its natural oscillation frequencies). The plate length influences vibration modes of the splitter plate, and the plate displacement is a function of Young's Modulus and its natural frequencies, see Lee and You (2013). An experimental study found that the plate displacement collapses on a single curve for different cases of dimensionless bending stiffness Shukla et al. (2013a). It is also well known that the oscillation amplitude and frequency of the plate tip is strongly affected by the combined effects of blockage and cylinder size Gallegos and Sharma (2016).

A second perspective, which also points out the importance of the FSI problems involving free surface deformation, comes to us from the traces left on the ocean by viscous wakes, and detected by radar observations of the ocean surface. There is no complete theoretical explanation of such traces owing to the great complexity of the problem. The linear problem of two-dimensional shear-flow/surface interaction was investigated by Triantafyllou and Dimas (1989), who found that two distinct regimes of unstable waves exist depending on the Froude and wavenumber. In Dimas and Triantafyllou (1994), the nonlinear evolution of the two branches of unstable waves are studied assuming an inviscid flow due to 
the high Reynolds numbers.

\subsection{Flat plates in cross-flow review}

Circular and square cylinders have been used as basic geometry for many kinds of research in fluid dynamics. From forces to vorticity evolution, those geometries are used as starting point of validation before going to different complex systems. Focusing on different numerical approaches for solving the wake behind a normal flat plate have been used in the past. Najjar and Balachandar (1998) studied numerically the 3D-periodic version of a flat plate in the laminar regime $(R e=250)$ in a single phase flow, and captured low-frequencies unsteadiness with periods approximately 10 times smaller than the primary shedding period. This behaviour was associated to two different flow states: an $\mathrm{H}$ regime of high mean drag and an $L$ regime of low mean drag. Narasimhamurthy and Andersson (2009) solved the same problem in the laminar regime using Direct Numerical Simulations (DNS) at a Reynolds number of 750 , exploring the coupling between the base pressure and the vortex formation process. Hemmati et al. (2016b) compared simulations of 3D-periodic flow against 3D flow, for a particular aspect ratio flat plate with sharp corners and $R e=1200$. The results they obtained, showed very different features in terms of wake dynamics, vortex structures, frequencies in the wake and averaged drag values. Apparently, the presence of side-edge shear layers suppressed the spanwise instabilities responsible for the 3 distinct flow regimes that were observed in the wake of 3D-periodic plates. A vortex peeling mechanism linked to the vortex detachments in the shear layers on the shorter sides, seems to appear only in 3D flows. Moreover, the peeling mechanism leads to the formation of interlocked vortex loops outside the base region. Another important finding was the single dominant shedding frequency observed in the wake, which indicated the two shear layers were rolling up at the same frequency at adjacent plate edges. In Hemmati et al. (2016a), the 3D-periodic normal flat plate was studied again using DNS at Reynolds numbers of 1200 and 2400, and a distinct period of reorganization $M$ was identified following regime $\mathrm{H}$.

In Hemmati et al. (2018) the same 3D-periodic problem is studied using DNS and Large Eddy Simulation (LES) for a extended Reynolds range up to $R e=1.5 \cdot 10^{5}$, where the author suggests that for $R e>1000$ the influence of Reynolds number on the drag and pressure coefficient as well as on the Strouhal number and the mean recirculation length, was limited.

Tian et al. (2014) studied numerically the 3D-periodic case using a LES model at turbulent Reynolds numbers of $1.5 \cdot 10^{5}$, in a plate with smooth corners. This work showed that there corners configuration and the reduction of the radius of curvature of the plate edges, heavily altered the flow patterns. It increased the mean and the fluctuating part of the drag and the lift forces on the plate, as well as the kinetic energy in the near wake. The work was complemented by Tian et al. (2012) and Najjar and Balachandar (1998) where the authors showed how their 2D simulations resulted in different hydrodynamic forces if compared 3D LES or experimental results. 
Although most of the studies performed with rectangular plates have been carried out without considering the free surface (a single phase flow), some exceptions are found in the literature. One of the numerical studies that includes free surface is Liu et al. (2016). This 2D RANS study, based on the $k-\omega$ SST model at Reynolds number of $5 \cdot 10^{4}$, considers the free surface influence on the plate dynamics for turbulent flows with free surfaces. Even at their highest submergence depth case, where the free surface influence is expected to be minimal or negligible, the authors showed different mean drag values when compared to those found in other numerical studies, in which the free surface was not taken into account Tian et al. (2014). Despite the geometry being different, it shows similar vortex dynamics and similar drag trends with depth, as in Miyata et al. (1990). When the cylinder is placed closer to the free surface, the drag force decreases and the dimensionless frequency increases.

Satheesh and Huera-Huarte (2019) found a relevant dependence on the aspect ratio and submergence depth, when they studied experimentally rigid plates of different aspect ratios, at Reynolds numbers of 3, 4.5 and $6 \cdot 10^{4}$, near the free surface. In general, they have showed how the drag force acting on the plate has a maximum value when near the free surface, as the gap flow modifies the wake reducing recirculation length. They also showed that the mean drag force was independent of Reynolds number, except when the aspect ratios of the plate (span to chord ratio) were in the range from 0.625 to 1.6 and the plate was near the free surface.

\subsection{Free surface influence review}

Most of the complex engineering applications involve the presence of the free surface. In the past, researchers have dealt with such problems numerically, using different simplifications, i.e. limiting geometries to 2D domains, using single phase models or assuming low Reynolds numbers that imply the absence of turbulence. Sheridan et al. (1997) and Reichl et al. (2005b) studied the influence of the free surface in a $2 \mathrm{D}$ submerged circular cylinder. They both showed the strong asymmetry created by the free surface in the vortex dynamics. These authors observed that if the free surface is taken into account, the Froude number defined as $F r=\frac{U}{\sqrt{g D}}$ where $U$ is the free stream velocity flow, $g$ is the gravity acceleration and $D$ is the cylinder diameter, is crucial in order to understand the problem. Other numerical methods such as Smooth Particle Hydrodynamics (SPH) were used by Bouscasse et al. (2017) who continued the work started by Reichl et al. (2005b), studying the wake behaviour at a Reynolds number of 180. In those works, different free surface deformations were observed depending on the Froude number, where supercritical to subcritical transitions take place in the near wake resulting in localized free-surface sharpening and wave breaking. Miyata et al. (1990) studied the fluid forces and the free surface influence on a submerged circular cylinder in turbulent regime $R e=4.96 \cdot 10^{4}$. Numerical simulations and experiments were performed showing a simultaneous decrease of the drag coefficient and an increase of the Strouhal number when the depth of the circular cylinder was gradually reduced. Malavasi 
and Guadagnini (2007) showed experimentally the influence on the hydrodynamic forces of the relative position of a rectangular cylinder, asymmetrically placed between the free surface and the channel floor. This effect had a large impact on the transverse force but barely affected the drag coefficient. 


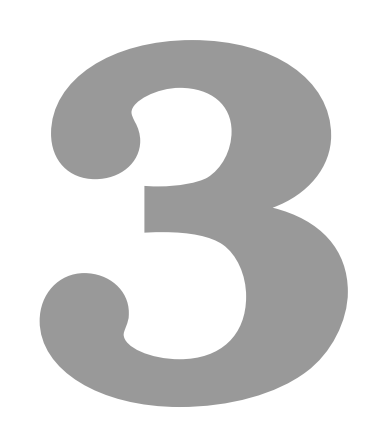

\section{Physical and numerical modelling}

\section{Contents}

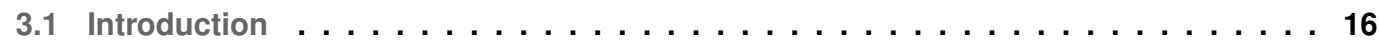

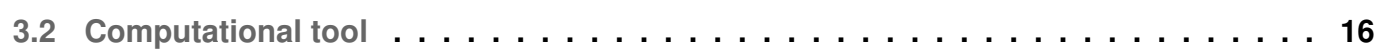

3.3 Fluid dynamic solver $\ldots \ldots \ldots \ldots \ldots \ldots \ldots \ldots \ldots \ldots$

3.4 Solid mechanics solver . . . . . . . . . . . . . . . . . 20

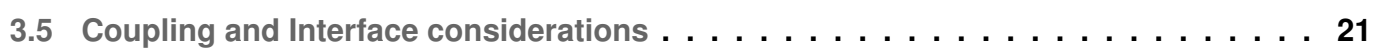

3.6 Dimensionless parameters . . . . . . . . . . . . . . . 21 


\subsection{Introduction}

In this chapter, a complete description of the numerical framework used is performed. This numerical setup was implemented in a computational tool 3.2 which is also described.

The aim of this thesis is the physical understanding of the complexity inside the problems described in chapters 4 and 5 and not its implementation in a numerical code. Nonetheless, from the readers' point of view, this chapter is crucial since most of the numerical assumptions are introduced.

In chapter 4, the fluid is solved with the incompressible Navier-Stokes equations in laminar regime while in chapter 5 a turbulent regime is assumed and modelled using Large-eddy simulation (LES).

When elastic solid bodies are part of the problem, see chapter 4, the solid is treated as hyperelastic and its motion is affected by the fluid pressure and shear effects. One important aspect to remark on the numerical modeling of the elastic bodies is the use of the finite volume method (FVM) in contrast with the frequently finite element method (FEM) method used in most commercial codes. In chapter 5 the solid is considered as rigid, therefore no displacements are allowed.

All the coupling and interface considerations will be explained in section 3.5, and will be later implemented in chapter 4 as part of a two way coupling system.

This chapter ends with the general definition of the dimensionless parameters considered in this thesis. These non dimensional numbers will adapted in each chapter to the particular characteristics of the geometry considered.

\subsection{Computational tool}

The first consideration is the use of the open source tool OpenFOAM 2018 as the computational CFD software. OpenFOAM is basically a $\mathrm{C}++$ library, that allows to create executables, known as applications. Applications can be splitted up in two: solvers that are used to solve a specific problem in continuum mechanics, and utilities, that are used to perform tasks that involve data manipulation.

OpenFOAM is supplied with preprocessing and postprocessing environments. Their interface are OpenFOAM utilities, thereby ensuring consistent data handling across all environments 3.1.

Finally, the versions used are: foam- extend 3.2 for FSI problems and OpenFOAM - dev when just fluid dynamics is involved. 


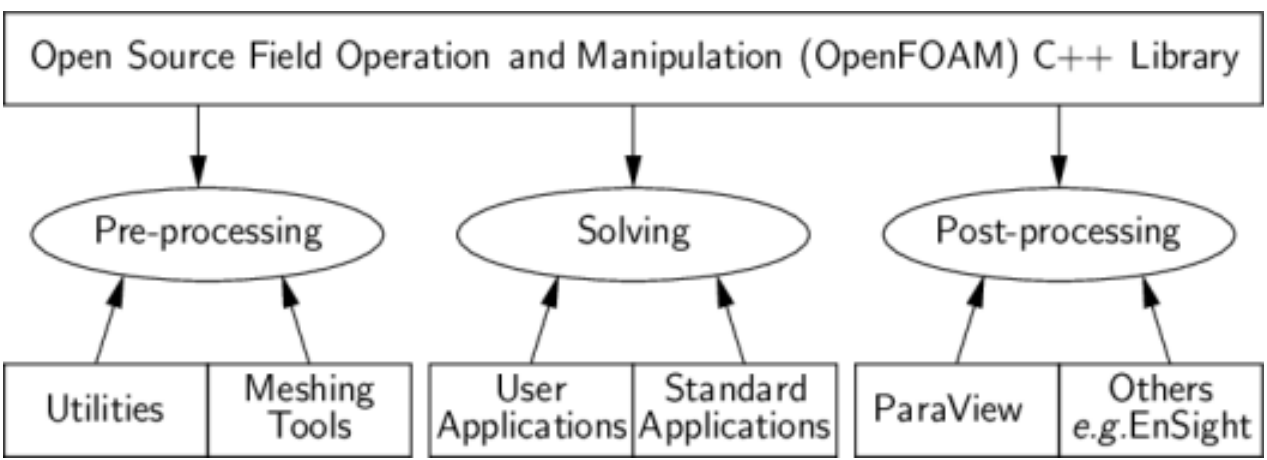

Figure 3.1: OpenFoam overview structure.

\subsection{Fluid dynamic solver}

\subsubsection{Laminar assumption}

The equations to be solved for the fluid part are the incompressible isothermal Newtonian NavierStokes equations. As the Reynolds numbers investigated are moderate, we will consider laminar regime, and consequently no turbulence models are included.

$$
\begin{aligned}
& \nabla \cdot \mathbf{v}=0 \\
& \frac{\vartheta(\rho \mathbf{v})}{\vartheta t}+\nabla(\rho \mathbf{v} \mathbf{v})=\rho \mathbf{g}-\nabla p+\nabla(\mu \nabla \mathbf{v})
\end{aligned}
$$

where $\mathbf{v}$ is the fluid velocity vector, $p$ is the pressure field, $\rho$ and $\mu$ the fluid density and viscosity. A transient PISO algorithm (Pressure Implicit Splitting of Operators), implemented in OpenFOAM 2018, was used to solve the unsteady Navier-Stokes equations. This algorithm,compared with the SIMPLE algorithm, contains an additional corrector step with one predictive step and two correction steps, see Versteeg H.K. (2007) and Oro (2012). For the time discretization, both an Euler and backward temporal second order discretization scheme were used, and very little differences were found. The convection term has been discretized using a second order upwind scheme.

\subsubsection{LES assumption}

For large enough Reynolds numbers, large-eddy simulation (LES) is used to model the subgrid scale. This technique solves large scale motions that contain most of the turbulent kinetic energy while small scale eddies are added with a subgrid scale (SGS) model to represent the effects of unresolved motions on the resolved scales. LES uses a spatial average of the transport equations using a filter of size $\Delta$, 
that separates the large scales solved and the small scales modelled.

In order to define a velocity and pressure field that assures the large fluctuations of the instantaneous velocity, the primitive variables of the Navier-Stokes equations are filtered providing a local mean of the turbulent flow. The filtered variable is defined as:

$$
\bar{f}(\vec{x})=\int_{\Omega} f\left(\vec{x}^{\prime}\right) G\left(\vec{x}, \vec{x}^{\prime}, \Delta\right) d \vec{x}^{\prime}
$$

where $G\left(\vec{x}, \vec{x}^{\prime}, \Delta\right)$ is the filtering function that depends on the cut-off width $\Delta$. Where $G\left(\vec{x}, \vec{x}^{\prime}, \Delta\right)$ must satisfy the following property:

$$
\int_{\Omega} G\left(\vec{x}, \vec{x}^{\prime}, \Delta\right) d \vec{x}^{\prime}=1
$$

the Cubic-root volume is used as length scale and given by:

$$
\Delta=c(\Delta x \Delta y \Delta z)^{1 / 3}
$$

where $c$ is a model coefficient that is equal to 1 . Therefore, the filtered incompressible Navier-Stokes equations for the filtered variables taking into account equations 3.1 and 3.2 are:

$$
\begin{aligned}
\nabla \cdot \overline{v_{i}} & =0 \\
\frac{\vartheta\left(\rho \overline{v_{i}}\right)}{\vartheta t}+\nabla\left(\rho \overline{v_{i} v_{j}}\right) & =-\nabla \bar{p}+\nabla\left(\mu \nabla \overline{v_{i}}\right)
\end{aligned}
$$

where $\nabla\left(\rho \overline{v_{i} v_{j}}\right)$ includes the filtering of the product of the variables. This is why in order to obtain a transport equation for the velocities, the term $\nabla\left(\rho \overline{v_{i}} \overline{v_{j}}\right)$ is added and subtracted in equation 3.7, resulting in:

$$
\frac{\vartheta\left(\rho \overline{v_{i}}\right)}{\vartheta t}+\nabla\left(\rho \overline{v_{i}} \overline{v_{j}}\right)=-\nabla \bar{p}+\nabla\left(\mu \nabla \overline{v_{i}}\right)-\nabla\left(\rho \overline{v_{i} v_{j}}\right)+\nabla\left(\rho \overline{v_{i}} \overline{v_{j}}\right)
$$

The last two terms are known as turbulent tensions of the subscales and are normally written as:

$$
\tau_{i j}=\rho\left[\overline{v_{i} v_{j}}-\overline{v_{i}} \overline{v_{j}}\right]
$$


where the term $\tau_{i j}$ is modelled and not computed in equation 3.9. Finally, the LES transport equations are:

$$
\begin{aligned}
\nabla \cdot \overline{v_{i}} & =0 \\
\frac{\vartheta\left(\rho \overline{v_{i}}\right)}{\vartheta t}+\nabla\left(\rho \overline{v_{i}} \overline{v_{j}}\right) & =-\nabla \bar{p}+\nabla\left(\mu \nabla \overline{v_{i}}\right)-\nabla \tau_{i j}
\end{aligned}
$$

The algebraic model chosen is that developed by Smagorinsky and Lilly (Smagorinsky (1963)), which computes directly the SGS stresses $\tau_{i j}$ from the filtered variables with the following formulation:

$$
\tau_{i j}-\frac{1}{3} \tau_{k k} \delta_{i j}=\mu_{s g s} \bar{S}_{i j}
$$

where Einstein convention is used for repeated indices. Furthermore, $\bar{S}_{i j}$ is the mean strain rate tensor $\bar{S}_{i j}=\frac{1}{2}\left(\frac{\vartheta \bar{v}_{i}}{\vartheta x_{j}}+\frac{\vartheta \bar{v}_{j}}{\vartheta x_{i}}\right), \delta_{i j}$ is the Kronecker's delta, and $\mu_{s g s}$ is the artificial viscosity calculated as:

$$
\mu_{s g s}=-2\left(C_{s} \Delta\right)^{2}|\bar{S}|
$$

where $|\bar{S}|=\left(2 S_{i j} S_{i j}\right)^{1 / 2}$ and $C_{s}=0.18$ are predefined values that result from assuming that the energy spectrum has an inertial range defined by the Kolmogorov $-5 / 3$ power law. An alternative model, known as Wall-adapting local eddy-viscosity (WALE) model Nicoud and Ducros (1999), was also used to compare the LES results and to quantify the dependence on the subgrid scale modelling.

A transient PISO algorithm (Pressure Implicit Splitting of Operators) implemented in OpenFOAM 2018 was used to solve the unsteady filtered Navier-Stokes equations (3.11). For the time discretization, an Euler scheme was used. The spatial scheme for interpolation are Gauss linear for the gradient, Gauss linear corrected for the Laplacian and Gauss linear Upwind for the divergence.

\subsubsection{Volume of Fluid consideration}

The Volume of Fluid (VOF) method is based on the idea of a scalar fraction function $\alpha$ defined locally on each cell of the mesh whose value depends on the fraction of volume occupied by each fluid. As the two fluids $A$ and $B$ are separated by the free surface, the value of $\alpha$ is zero when a cell is filled with fluid $\mathrm{A} ; \alpha=1$ when the cell is full of fluid $\mathrm{B}$; and $0<\alpha<1$ when a cell is shared between both fluids and the interface divides the cell. The equation to model the volume fraction of one phase $\alpha$, without mass 
sources or mass transfers between phases, is the following:

$$
\frac{\partial \alpha}{\partial t}+\mathbf{v} \cdot \nabla \alpha=0
$$

Once the fraction function $\alpha$ is known, the local values of the density and viscosity $\rho$ and $\mu$ are computed accordingly for each finite volume.

\subsection{Solid mechanics solver}

For an arbitrary elastic body of volume $\Omega$, bounded by surface $\Gamma$ with unit normal $\mathbf{n}$, the conservation of linear momentum in integral form is given by

$$
\underbrace{\frac{D}{D t} \int_{\Omega} \rho_{s} \mathbf{u}_{s} d \Omega}_{\text {Acceleration }}=\underbrace{\oint_{\Gamma} \mathbf{n} \cdot \sigma d \Gamma}_{\text {Surface Forces }}+\underbrace{\int_{\Omega} \rho_{s} \mathbf{b} d \Omega}_{\text {Body Forces }}
$$

where $\mathbf{u}_{s}$ is the solid velocity, $\sigma$ is the stress tensor, $\rho_{s}$ is the solid density, and $\mathbf{b}$ is the body force per unit mass. A constitutive relation of a solid material essentially relates the material stresses to the material strains. In the current study, the body is assumed to be elastically isothermal, therefore thermal and plastic effects are neglected. An elastic solid undergoing large strains and large rotations is governed by the St. Venant-Kirchhoff hyperelastic constitutive relation 3.16. These equations are solved using the finite volume method.

$$
\mathbf{S}=2 \mu \mathbf{E}+\lambda(\mathbf{E}) \mathbf{I}
$$

where $\mathbf{E}$ is the Lagrangian tensor and $\mathbf{S}$ is the second Piola-Kirchhoff stress tensor.

$$
\mathbf{E}=\frac{1}{2}\left(\nabla u_{s}+\nabla u^{T}+\nabla u \nabla u^{T}\right)
$$

The Lame coefficients are $\mu$ and $\lambda$ are related with the Young's modulus $E$ and the Poisson $\nu$. They are defined as:

$$
\mu=\frac{E}{2(1+\nu)}
$$




$$
\lambda=\frac{\nu E}{(1+\nu)(1-2 \nu)}
$$

\subsection{Coupling and Interface considerations}

The coupling algorithm that has been used to simulate the solid and fluid interaction is the Aitken algorithm. Based on our experience, this algorithm offers robustness and good convergence. Others algorithms such as IQN-ILS Degroote et al. (2009) could be used, but they are less robust.

The contact interface between the solid and the fluid must satisfy two basic conditions. The kinematic condition imposes the contact points between the fluid and the solid to have the same velocity. The second condition is the dynamic condition, which states the stress equilibrium between the fluid and the solid at the contact surfaces. These are:

$$
\mathbf{v}_{f}=\mathbf{v}_{s}
$$

$$
\sigma^{f} \cdot \mathbf{n}=\sigma^{s} \cdot \mathbf{n}
$$

where $\sigma^{\mathbf{f}}$ and $\sigma^{\mathbf{s}}$ are the stress tensors associated to the fluid and solid respectively. The constant $z$ planes are considered to be symmetry planes, and the splitter plate in displacements and velocities interacts with the fluid mesh points. The left part of the structure is considered to be cantilevered and no deformations or displacements are allowed in the $Z$ direction.

\subsection{Dimensionless parameters}

Along this thesis we consider the general case where two different fluid phases are present and an averaged upstream velocity $\bar{U}$ interacts with the structure. The solid is submerged in the bottom phase and when no rigid solid is considered, we denote density as $\rho_{f}^{b}$ and viscosity as $\mu_{f}^{b}$. The top phase has density $\rho_{f}^{t}<\rho_{f}^{b}$ and viscosity $\mu_{f}^{t}$. The Reynolds number $(R e)$, the Froude number $(F r)$, the Cauchy number $(C y)$, the mass ratio $(M)$, Strouhal number $(S t)$ and the geometrical parameter $h / D$ that controls 
the free surface influence.

$$
R e=\frac{\rho_{f}^{b} \bar{U} D}{\mu_{f}^{b}} \quad C_{y}=\frac{\rho_{f}^{b} \bar{U}^{2}}{E} \quad F r=\frac{\bar{U}}{\sqrt{g D}} \quad M=\frac{\rho_{f}^{b}}{\rho_{s}} \quad S t=\frac{f D}{\bar{U}}
$$

Note that the Cauchy number and the mass ratio are only used in FSI problems. Related to the Cauchy number, other authors such as Shukla et al. (2013a) use the non-dimensional bending stiffness parameter $k_{B}=2 E I / \rho_{f}^{b} U^{2} l^{3}$ instead of $C y$, where $E I$ is the flexural rigidity of the plate. The relation between both parameters in 2D problems is $k_{B}=\frac{1}{6 C_{y}}\left(\frac{e}{l}\right)^{3}$.

In the Strouhal number, $f$ is the lift force frequency. Because the fluid properties are fixed, the non dimensional ratios $\rho^{b} / \rho^{t}$ and $\mu^{b} / \mu^{t}$ will not be changed throughout each work in this thesis, 4 and 5 . The hydrodynamic forces acting on the structure, $F_{x}$ and $F_{y}$ respectively, are non-dimensionalized in the form of drag $C_{D}$ and lift $C_{L}$ coefficients.

$$
C_{D}=\frac{F_{x}}{\frac{1}{2} \rho \bar{U}^{2} D^{2}} \quad C_{L}=\frac{F_{y}}{\frac{1}{2} \rho \bar{U}^{2} D^{2}}
$$

Notice that in 4 and 5 these terms will be considered again along with their correspondingly scaled parameters. 


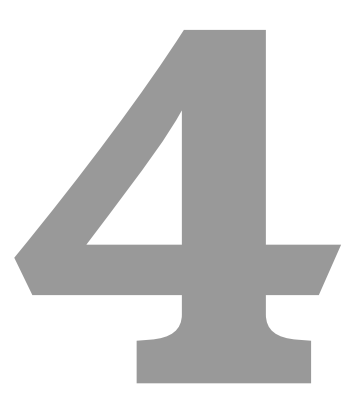

\section{Laminar simulation of the effect of the} free surface on a flexible splitter plate in the wake of a stationary cylinder

\section{Contents}

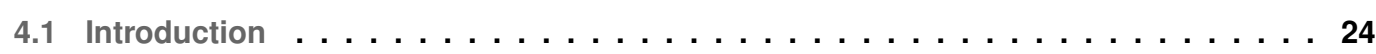

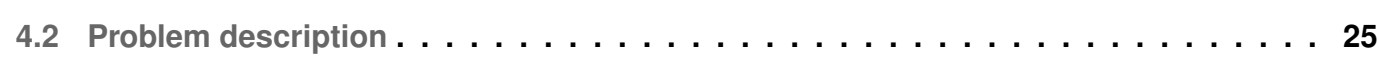

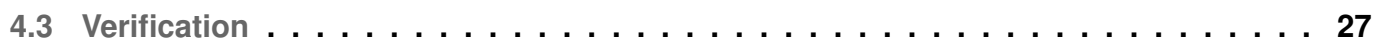

4.4 Chapter conclusions $\ldots \ldots \ldots \ldots \ldots \ldots \ldots \ldots \ldots \ldots \ldots \ldots$ 


\subsection{Introduction}

This chapter shows a problem that combines on one hand the complexity of a viscous flow around a bluff body that contains a deformable structure on the wake, and on the other hand the complexity introduced by the presence of free surface. The combination of FSI phenomena and free surface deformation makes this problem very attractive from a computational point of view.

This work evaluates the influence of the sea surface on a typical fluid-structure interaction device such as that investigated by Turek et al. (2010). The present numerical study also shows an extensive investigation of effects of the dominant non dimensional numbers on the drag and lift of a cylinder and deformation of the plate. The computational methodology is validated in one benchmark case: flow over a circular cylinder with a splitter plate such as in the case studied by Turek et al. (2010), and a second case where free surface is added to the problem, and consequently new non-dimensional numbers appear. Two publications Diaz-Ojeda et al. (2018) and Díaz-Ojeda et al. (2019) have arisen from this work.

A schematic representation of the two-way interaction between an elastic structure and an incompressible flow problem appears in figure 4.1. The problem is considered to be two dimensional, therefore no deformations or displacements are allowed in the out of plane. The flow comes from the left of the rigid cylinder. The structure is deformed by the pressure and the viscous forces created around the splitter plate due to the vortex shedding produced by the cylinder. In those cases where two immiscible Newtonian fluids are involved in the problem, gravity forces and a free surface are added to the problem. In that situation, the structure formed by a cylinder with diameter $D$ and a cantilever splitter plate of length $l$ and width $e$, is submerged a distance $h$ below the free surface. Similarly to Reichl et al. (2005b), the depth $h$ is measured from the free surface to the top of the cylinder. Since free surface is present, gravity $g$ is applied over the domain. OpenFOAM version foam-extend 3.2, was used to solve this FSI problem as introduced in 3 . This code contains a fluid solver, an elastic solid solver and the possibility of coupling both parts.

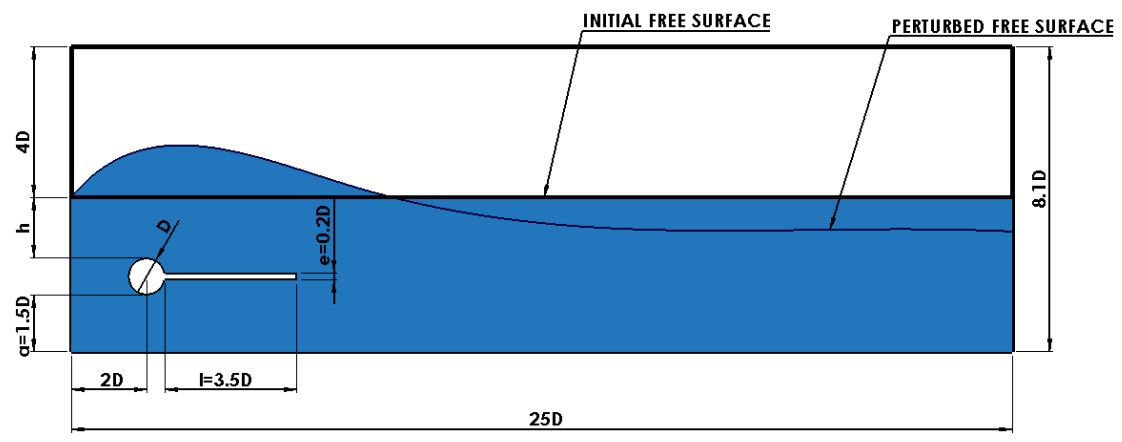

Figure 4.1: Problem set up and main geometric parameters 


\subsection{Problem description}

In this work two cases have been studied and compared. In the first, the geometry and the parameters proposed by Turek et al. (2010), were studied. The cases pose two typical two-way FSI problem where an elastic rectangular structure is deformed by the pressure and shear forces created in the surroundings of the splitter plate due to the effect of the flow separated around the cylinder. The geometry used in the first case has similar dimensions to the one presented in previous literature, where the rectangular domain has $4.1 \mathrm{D}$ and $25 \mathrm{D}$ with the thickness $0.5 \mathrm{D}$, with the cylinder diameter being $D=0.1$ $\mathrm{m}$. The circular cylinder has its center placed at point $\left(x_{c}, y_{c}\right)=(0.2,0.2) \mathrm{m}$. The dimensions of the flexible structure are $3.5 D$ by $0.2 D$. A test point named $A$ is defined at the position $x=6 D, y=2 D$, and will be considered as the reference point for measuring the displacement of the splitter plate. Once the comparison against Turek et al. (2010) has been completed, the following step will be the study of the same problem, but adding free surface and varying the dimensionless numbers. As shown in figure 4.1, an additional rectangular domain $25 D$ long, $4 D$ wide and filled with air has been added on the top of the previous geometry proposed originally by Turek et al. (2010).

The material considered for the flexible structure, is a combination of two rubber-like materials such as polybutadiene and polypropylene with density $\rho_{s}$, Young's modulus $E$ and Poisson coefficient $\nu^{s}$. Due to the very low stiffness of the materials used here, large deformations of the structure are possible.

Assuming that the geometry and fluid property ratios are fixed, the non dimensional parameters $e / D$, $l / D, \rho_{f}^{b} / \rho_{f}^{t}=1000$ and $\mu_{f}^{b} / \mu_{f}^{t}=67.57$ will not be changed throughout this work.

In the verification case, the benchmark from Turek and Hron (2007) or Turek et al. (2010), has an inlet velocity with a parabolic profile and a mean velocity $\bar{U}=2 \mathrm{~m} / \mathrm{s}$, defined as

$$
U=\frac{3}{2} \bar{U} \frac{y(H-y)}{\left(\frac{H}{2}\right)^{2}}
$$

where $H=a+h+D$. For the second multiphase case, where the cylinder and the elastic plate are both submerged under the denser fluid in the presence of a gravity field and the free surface, the parabolic profile will be changed for a mean velocity $\bar{U}=2 \mathrm{~m} / \mathrm{s}$.

\subsubsection{Mesh description}

Different meshes have been studied in order to accurately measure the forces acting on the structure, and that has required a high number of cells in the mesh. In order to capture the tension and compression zones, it is absolutely necessary to use more than 4 cell in the transverse section of the splitter plate even having a very small thickness, see figure 4.2 where the solid computational domain is 


\begin{tabular}{|c|c|c|c|}
\hline Mesh & Cells structure domain & Cells fluid domain & $A_{h} / D^{2}$ \\
\hline 1 & 1000 & 11554 & $6.09 e-3$ \\
\hline 2 & 1250 & 17924 & $3.06 e-3$ \\
\hline 3 & 1500 & 21774 & $2.03 e-3$ \\
\hline Turek and Hron (2007) & 19488 & & \\
\hline
\end{tabular}

Table 4.1: Number of cells of each subdomain, fluid and structural and cell size close to the beam $\left(A_{h} / D^{2}\right)$. The total number of cells (structure+fluid) in Turek and Hron (2007) mesh is also included for reference.

\begin{tabular}{ccccccc}
\hline Mesh & $\frac{\Delta t \bar{U}}{D}$ & $X / D$ & $Y / D$ & $\frac{f_{x} D}{\bar{U}}$ & $\frac{f_{y} D}{\bar{U}}$ & \% relative error in Y/D \\
\hline Turek and Hron (2007) & $1 \mathrm{e}-2$ & 0.0285 & 0.3563 & 0.5375 & 0.2686 & - \\
1 & $1 \mathrm{e}-2$ & 0.02495 & 0.3607 & 0.5375 & 0.2686 & 1.01 \\
2 & $1 \mathrm{e}-2$ & 0.02684 & 0.3662 & 0.5375 & 0.2686 & 1.02 \\
3 & $1 \mathrm{e}-2$ & 0.02853 & 0.3676 & 0.5375 & 0.2686 & 1.03 \\
3 & $2 \mathrm{e}-2$ & 0.02765 & 0.3682 & 0.5375 & 0.2686 & 1.03 \\
\hline
\end{tabular}

Table 4.2: Comparison between different meshes and different time steps with the benchmark case

presented. Three different structured meshes were used for the purpose, see figure 4.3 as an example. In order to validate mesh convergence, the number of cells on the cylinder and on the splitter plate have been varied. As shown in figure 4.4, the number of cells in the $x$ and $y$ boundaries of the geometry should match the number of cells in the splitter plate and cylinder. The same happens in the cases in which the air domain is added to the top. The number of cells for the solid and fluid parts of the three different meshes are listed in Table 4.1. The horizontal displacement of point $A$, initially at $(6 D, 2 D)$ referred to as $X$, is an order of magnitude smaller than the vertical displacement $Y$. A mesh convergence process based on the non dimensional tip displacement is presented in Table 4.2.

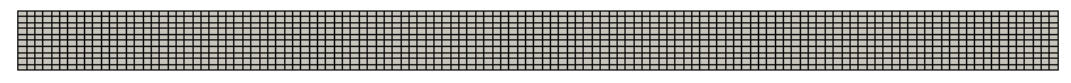

Figure 4.2: Schematic of the solid computational domain.

A time step convergence process has been carried out using different time steps. Time steps 


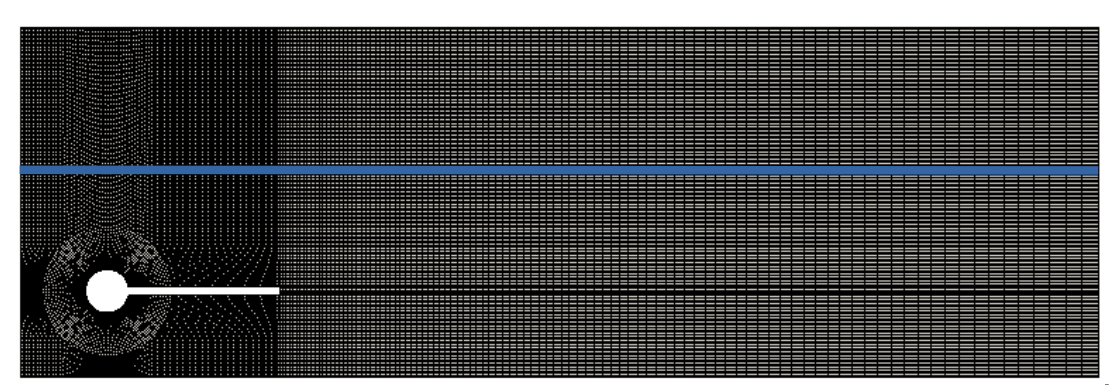

Figure 4.3: Structure and general view of the mesh 2 for the free surface case. The blue line indicates the initial free surface when two fluids are simulated.

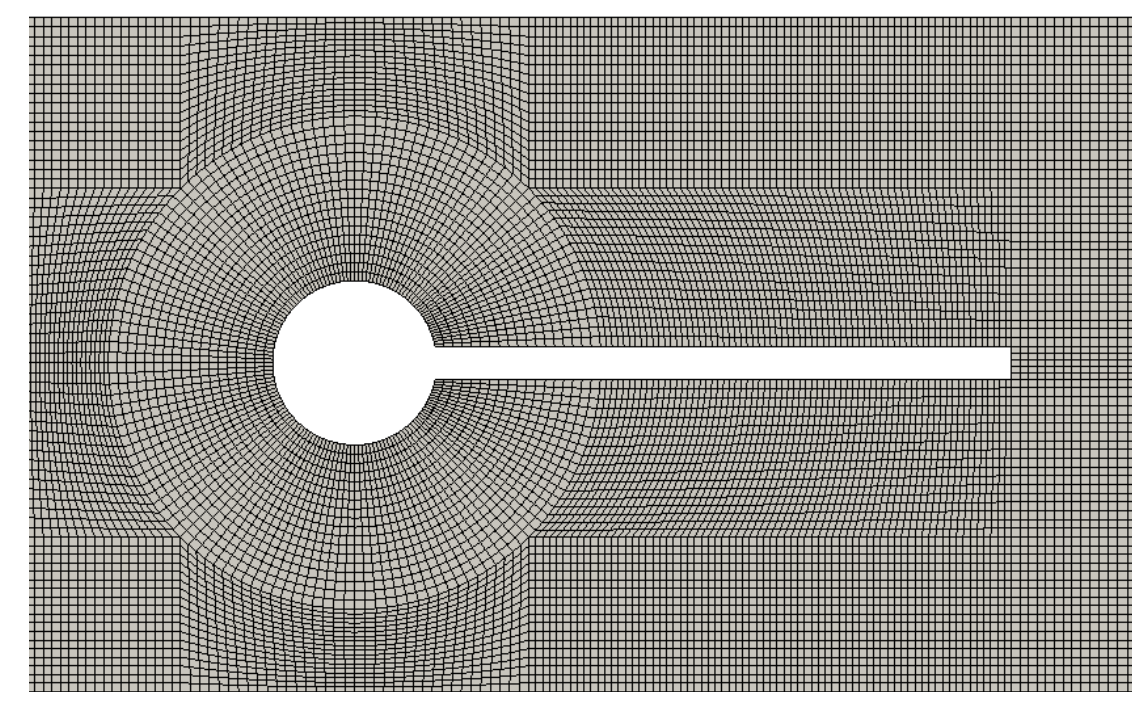

Figure 4.4: Zoom of the mesh 2 over the cylinder and splitter plate.

$\frac{\Delta t \bar{U}}{D}=2 \times 10^{-2}$ and $\frac{\Delta t \bar{U}}{D}=10^{-2}$ have been considered for both the structure and fluid parts, showing differences under $1 \%$, see Table 4.2. It is worth stressing that the same time step has been used for both the structure and fluid parts.

\subsection{Verification}

Before studying the influence of the free surface on the case described in section 4.2, some previous tests have been performed to assess the validity of the numerical procedures. The validation cases are the ones proposed by Turek et al. (2010), which in fact, have been studied by other authors such as Gallegos and Sharma (2016) and Bhardwaj and Mittal (2012). Two different cases, FSI 2 and FSI 3 from now on, have been run. The main results monitored are the displacements of the tip of the splitter plate (point $A$ ), the total drag and lift forces acting on the structure (formed by both the cylinder and the splitter plate) and the dominant frequency of the splitter plate oscillations. 


\begin{tabular}{ccc}
\hline Parameter & FSI 2 & FSI 3 \\
\hline$\rho^{s}\left[\frac{\mathrm{kg}}{\mathrm{m}^{3}}\right]$ & $10^{4}$ & $10^{3}$ \\
$\nu^{s}$ & 0.4 & 0.4 \\
$\rho^{f}\left[\frac{\mathrm{kg}}{\mathrm{m}^{3}}\right]$ & $10^{3}$ & $10^{3}$ \\
$\nu^{f}\left[\frac{\mathrm{m}^{2}}{\mathrm{~s}}\right]$ & $10^{-3}$ & $10^{-3}$ \\
$\bar{U}\left[\frac{\mathrm{m}}{\mathrm{s}}\right]$ & 1 & 2 \\
$E[P a]$ & $1.4 \cdot 10^{6}$ & $5.6 \cdot 10^{6}$ \\
\hline$R e$ & 100 & 200 \\
$C y$ & $7.14 \cdot 10^{-4}$ & $7.14 \cdot 10^{-4}$ \\
$M$ & 0.1 & 1
\end{tabular}

Table 4.3: Parameters used in the FSI2 and FSI3 validation cases

\subsubsection{Main verification cases (FSI2 and FSI3)}

The two different sets of parameters for cases FSI2 and FSI3, used for the validation exercise as in Turek et al. (2010) appear detailed in Table 4.3. The case presented in Turek et al. (2010) is based on a single fluid arrangement. The differences between cases FSI2 and FSI3 are therefore, the elastic properties of the solid, the average inflow velocity and the density of the elastic part, which clearly affects the inertial forces acting on the moving plate, this last property being the most significant difference between both test cases. The time-varying displacement of tip of the plate follows a sinusoidal pattern. Although the vertical displacement is dominant (see figure 4.5), the horizontal displacement of the tip has been also compared to the results available in the literature.

The amplitudes and frequencies of the horizontal and vertical displacement of point $A$ in case FSI3, appear in table 4.4 for comparison with the results obtained by Turek et al. (2010). In all the results presented in this work, the peak amplitude of the vertical displacement $Y$ has been used and compared to the value obtained using $\sqrt{2}$ of the root mean square (RMS) of the signal, showing practically no difference. The results presented by Turek et al. (2010) derive from a convergence test based on the number of cells in the mesh and the time step used for the computations. In the work presented here, for comparison purposes, results are derived from a mesh with a similar number of cells and time step. These are also similar to those used by Pisacreta (2017). Table 4.4 shows that no significant differences 


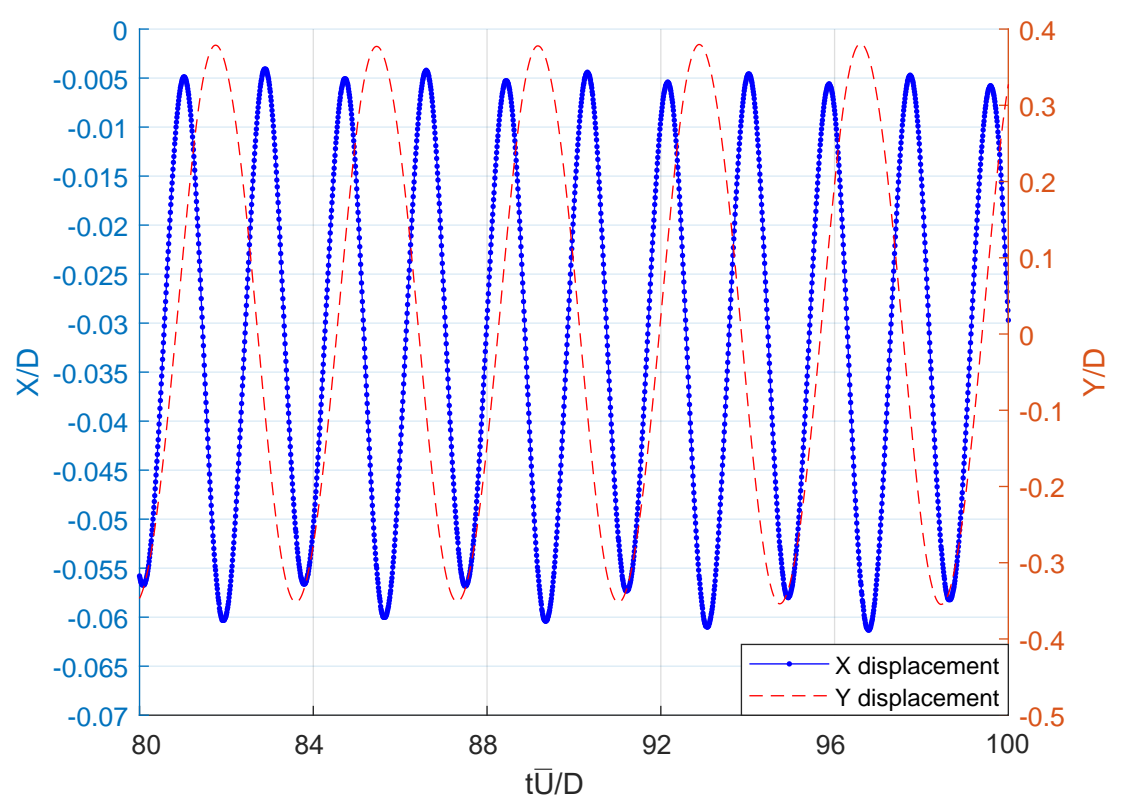

Figure 4.5: Horizontal and vertical time-varying displacements of point $A$

have been found. In the second validation case FSI2, results are compared to the work by Turek and Hron (2007), and additionally to the results found in Gallegos and Sharma (2016), obtained using the commercial software ANSYS.

\begin{tabular}{ccccc}
\hline Benchmark case FSI3 & X/D & Y/D & $\frac{f_{x} D}{\bar{U}}$ & $\frac{f_{y} D}{\bar{U}}$ \\
\hline Turek et al. (2010) & 0.0285 & 0.3563 & 0.5375 & 0.2686 \\
Pisacreta (2017) & 0.0252 & 0.3516 & 0.5495 & 0.2705 \\
Present work & 0.0268 & 0.3662 & 0.5375 & 0.2686
\end{tabular}

Table 4.4: Comparison between the amplitudes and frequencies of the horizontal and vertical displacements of point $A$ (splitter plate tip), obtained by Turek et al. (2010) and Pisacreta (2017), for case FSI3.

Figure 4.6 shows the instantaneous vorticity fields around the cylinder and how the vortex shedding induces the periodic vibration of the splitter plate. The vorticity field is shown in dimensionless form, defined as $\omega_{z}^{*}=\frac{\omega_{z} D}{U_{\infty}}$, where $\omega_{z}$ is the out of plane vorticity. The diameter of the cylinder determines the size of the wake structures generated, and consequently it influences the vertical amplitude of the plate. The shear layers appear elongated and after separating from the cylinder surface, they reattach at the surface of the splitter plate, which avoids their interaction as in the classical vortex street formation. After a transient flow determined by the initial conditions where the plate exhibits little deformation, the 
CHAPTER 4. LAMINAR SIMULATION OF THE EFFECT OF THE FREE SURFACE ON A FLEXIBLE SPLITTER PLATE IN THE WAKE OF A STATIONARY CYLINDER

\begin{tabular}{ccccc}
\hline Benchmark FSI2 & X/D & Y/D & $\frac{f_{x} D}{\bar{U}}$ & $\frac{f_{y} D}{\bar{U}}$ \\
\hline Turek and Hron (2007) & 0.1203 & 0.787 & 0.38 & 0.20 \\
Gallegos and Sharma (2016) & 0.1192 & 0.790 & 0.382 & 0.196 \\
Present work & 0.1057 & 0.778 & 0.39 & 0.1953 \\
\hline
\end{tabular}

Table 4.5: Comparison between of the amplitudes and frequencies of the horizontal and vertical displacements of extreme plate point $A$ and the Hron-Turek results Turek et al. (2010) for case FSI2.

motion of the plate reaches a periodic self-sustained oscillation with practically constant amplitude. The deformation of the plate can be seen in figure 4.7 , where eight different deflected shapes taking place during a full oscillation cycle appear superimposed. The deformations resemble those similar to the second mode of vibration of a cantilevered beam. The results in Tables 4.4 and 4.5, show how there is an accordance between the computations presented here and the results by Turek et al. (2010) and Pisacreta (2017).

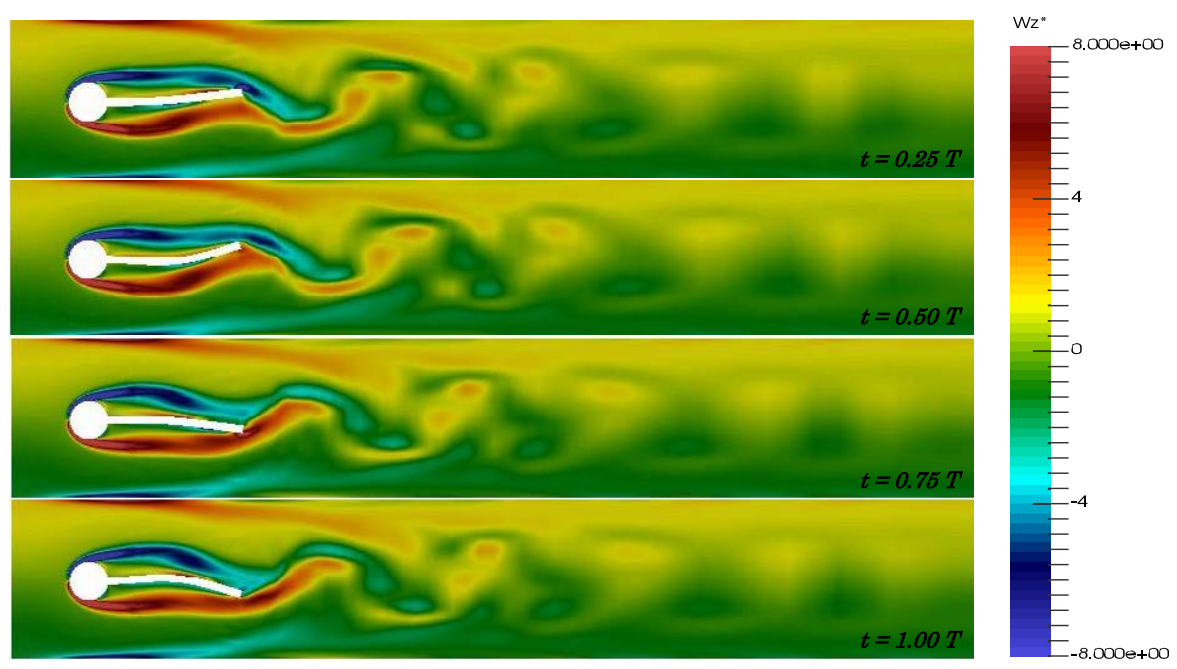

Figure 4.6: Instantaneous vorticity snapshots during a complete vortex shedding cycle of period $T$. Clockwise vorticity appears in blue (down to $\omega_{z_{\min }}^{*}=-8$ ) and counter-clockwise in red (up to $\omega_{z_{\max }}^{*}=8$ ). Vorticity is presented in dimensionless form, defined as $\omega_{z}^{*}=\frac{\omega_{z} D}{U_{\infty}}$

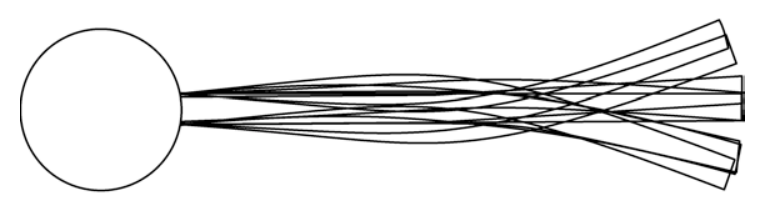

Figure 4.7: Superimposed view of the deflections of the splitter plate 
Notwithstanding, the FSI3 benchmark case is highly sensitive due to the great difficulties in obtaining a numerically stable solution. Phenomena such as inertial effects and mesh distortion must be avoided during the fluid and the structural coupling, especially during the transient part of the simulation. The integration of the different schemes must be performed in such a way that both solvers accept the solution provided by the other, and generate a coherent solution. The number of coupling iterations required to obtain convergence between both codes depend basically on how much the beam deforms. Excessive time steps imply large deformations and distortions in the mesh structure that yield errors during the calculation. During the transient part, where unexpected inertial effects might appear, the use of a very large number of internal iterations for fluid-structural coupling could be inefficient and divergent. Regarding the mesh distortion problems that might appear when parts of the structure suffer large accelerations, we must clarify that OpenFOAM-extend does not implement any re-meshing algorithm. The only possibility when the deformation of the mesh is excessive is the implementation of a quadratic or linear Laplace solver that smooths the node positions and prevents inconsistent cells.

\subsubsection{Sensitivity analysis of the validation cases FSI2 and FSI3}

In order to test the sensitivity of these results to the particular parameters and boundary conditions selected for cases FSI2 and FSI3, five additional cases were also studied. All of them have the same fluid and solid properties as case FSI3. The additional cases are:

- Case 1. Corresponds to the FSI 3 Turek et al. (2010), but using uniform inflow profile $U / \bar{U}=1$.

- Case 2. Same as Case 1, but using a rigid splitter plate.

- Case 3. Similar to FSI3 Turek et al. (2010), but using a rigid splitter plate.

- Case 4. The top wall is set at $10.1 D$ from the cylinder centre, and the bottom wall is set at $6 D$ from the cylinder center. Uniform inflow profile $U / \bar{U}=1$.

- Case 5. The top wall is set at $10.1 D$ from the cylinder centre, and the bottom wall is set at $2 D$ from the cylinder center. Uniform inflow profile $U / \bar{U}=1$.

In case 1 , the parabolic inflow is substituted by a uniform flow equal to the average value of the parabolic case. In the second and third cases, the elastic plate is replaced by a rigid one to measure the variation of the forces on the structure. Finally, in the last two cases the geometry is modified in order to evaluate the influence of blockage. In case 4 both walls are separated from the structure, and in case 5 only the bottom wall is separated while the other remains at the same position as in FSI3.

The amplitude of the vertical displacement of the tip in cases 1, 4 and 5 appears in figure 4.8. Using a uniform profile instead of the parabolic one used in FSI3 creates less deformation on the plate. When comparing case 1 with cases 4 and 5 , where the width of the channel is enlarged, we observe that the 
blockage effect of the top and bottom walls reduces the plate motion. This effect is inferred from the results presented in Zhang and Shi (2016), where the blockage effect was studied in a rigid version of the structure presented here. They showed that blockage clearly affects the frequency of the vortex shedding behind the structure, as well as the amplitude oscillation of the lift coefficient. Drag forces appear in figure 4.8 for all cases studied. According to the values obtained, there is not an important drag difference between the elastic and the rigid plate versions, with the FSI 3 and case 3 having similar values when the inflow is parabolic, or cases 1 and 2 for a uniform inflow. When the distance between the horizontal walls and the structure is enlarged, and consequently the blockage effect is reduced, the drag is also reduced with respect to the other cases. The dependence of the drag coefficient on the blockage effect was also studied by Zhang and Shi (2016). Some of the results obtained in this validation exercise, will be used as reference values in section A.3, where the free surface is added to the problem.
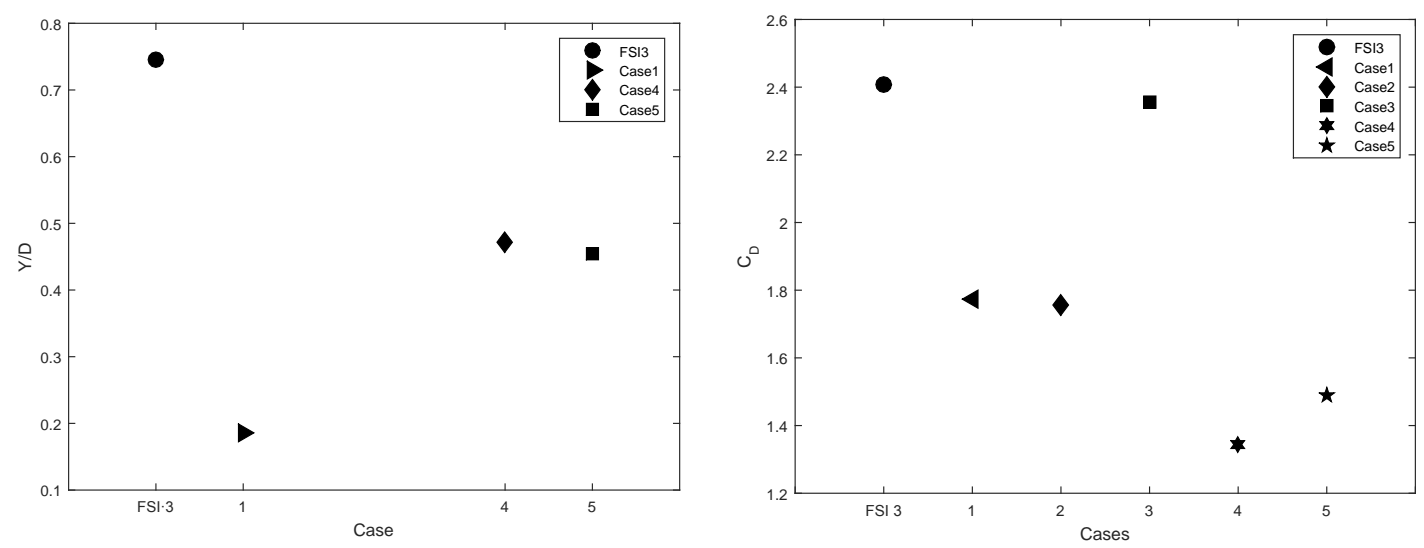

Figure 4.8: Comparison of the amplitude displacement (left) and drag force coefficient (right) for some the additional cases. Case 0: Turek case FSI 3. Case 1: Turek case FSI 3, but using uniform inflow profile $U=2$. Case 2: Case 1 with a rigid splitter plate. Case 3: Turek case FSI 3, but using a rigid splitter plate. Case 4: The top wall is set at $10.1 \mathrm{D}$ from the cylinder center, and the bottom wall is set at $6 \mathrm{D}$ from the cylinder center. Uniform inflow profile $U=2$. Case 5: The top wall is set at $10.1 D$ from the cylinder center, and the bottom wall is set at $2 D$ from the cylinder center. Uniform inflow profile $U / \bar{U}=1$.

With the numerical procedures fully verified, in this section the free surface and the gravity field are added to the problem. The goal is to investigate the effect of this discontinuity on the splitter plate dynamics and its excitation. In order to have a reference framework, the structure is analysed using fluids with the same characteristics as those used for the benchmark cases, namely glycerine and air at the upper part of the domain, see properties in the FSI3 case Table 4.3 and density and viscosity ratios in section 4.2. The non-dimensional parameter $h / D$ will be increased from 0.3 up to 1.6, where the distance between the upper part of the cylinder and free surface (at rest), is equal to the distance to the top wall in the benchmark cases.

The effects of fluid viscosity (Reynolds number), splitter plate stiffness (Cauchy number) and the 
gravity field (Froude number) on the dynamics and the excitation are studied in detail in order to provide a complete perspective of the problem. The mass ratio $M$ (structural mass upon displaced fluid mass) will be kept constant and equal to 1 , for all cases so the influence of buoyancy is kept away from the problem.

\subsubsection{Splitter plate deformation in the presence of free surface}

Initially the study is focused on the study of the effect of the distance to the free surface on the deformations of the splitter plate. Variations in the Reynolds number (fluid viscosity), Cauchy number (splitter plate flexural stiffness) and Froude number (gravity field) are introduced at the same time as varying $h / D$. Figure 4.9 shows the typical time series of the displacement of the tip of the splitter plate in one of the numerical experiments, run with $R e=200, F r=2, C y=7.14 \cdot 10^{-4}$ and $h / D=0.45$. Three time windows can easily be identified in the figure. First, the splitter plate deforms until it reaches its equilibrium position, in this case at a $t \sim 4 s$, due to the presence of the free surface which implies absence of symmetry in the vertical direction. Afterwards, the vortex shedding starts to change the pressure field leading to very small oscillations $(4 s<t<12 s)$ until it reaches a self-sustained oscillation with constant amplitude for $t>12 s$.

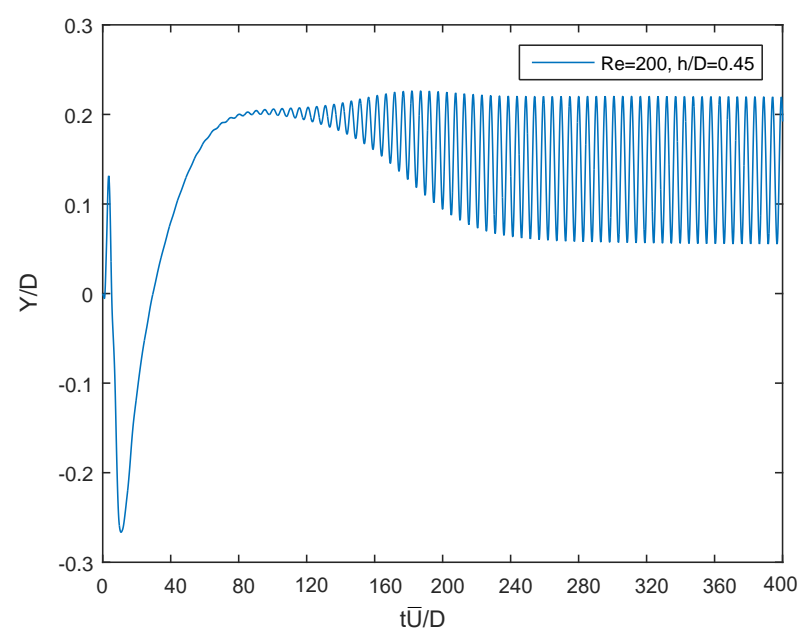

Figure 4.9: Evolution of vertical displacement of the tip of the plate corresponding with Reynolds number $R e=200$, Froude number $F r=2$, Cauchy number $C y=7.14 e-4$ and $h / D=0.45$.

Another important aspect is the influence of the proximity to the wall $(a / D)$. The tip amplitudes for a run with parameters $h / D=1.6, R e=200$ and $C y=7.14 \cdot 10^{-4}$ appear in figure 4.10. The figure allows to conclude that the tip amplitude barely changes if $a / D$ is over a certain value. 


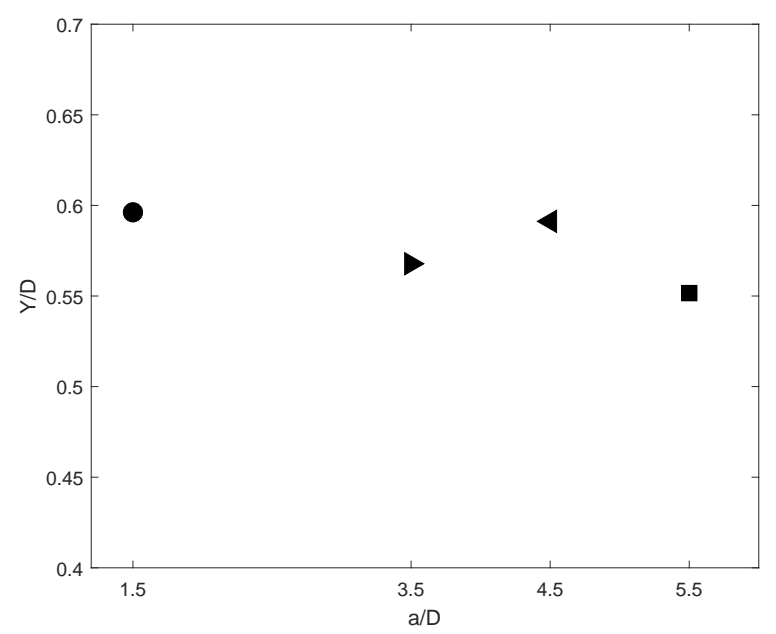

Figure 4.10: Vertical amplitudes of the plate tip versus distance to the bottom wall $a / D$. The Froude $F r=2$ and the Cauchy numbers $C y=7.14 \cdot 10^{-4}$ are kept fixed.

\subsubsection{A Effect of Reynolds number}

A series of simulations were carried out changing the Reynolds number in order to understand the influence of this parameter on the plate dynamics. The physical magnitude that was used to change the Reynolds number is the kinematic viscosity. Here, the rest of the dimensionless numbers in the problem, such as the Cauchy and the Froude number, will be kept constant with the values: $C y=7.14 \cdot 10^{-4}$ and $F r=2$. The $R e$ is varied from a value of 100 , with no tip displacements observed, to a value of 1000 . Note that with a $R e=1000$, if a fine mesh is used, turbulence modelling can be avoided, see 4.3.3.A for further information about turbulence in the present work. In figure 4.11, the vertical displacement of the tip of the splitter plate (point $A$ ) is represented against depth, for the different Reynolds numbers investigated. One of the reference cases from section 4.3.2, Case 4 with uniform inflow velocity $U / \bar{U}=1$ and $R e=200$ but with structure immersed in a single fluid (properties defined in the case FSI3 Table 4.3), has been included for comparison. This reference case is indicated in the figure using a horizontal dashed line. Re appear indicated using symbols.

The plot shows how there is a critical Reynolds number within the interval $R e=[100,150]$, at which the tip motion starts. In all cases, increases in Reynolds number result in increases of the splitter plate oscillations. This trend was also observed in the validation case FSI3 Pisacreta (2017). In fact the plot shows how amplitude in all curves increase monotonically with depth until a point at which it saturates, and depth starts to have small influence on it. For example, in the case of a $R e=200$, it is around an $h / D$ of 1.1. The growth of the amplitude with $h / D$ is a consequence of the asymmetry created by the gap flow between the free surface and the structure, that ultimately modifies the vorticity and pressure distributions in the wake of the system. Analogously to the rigid plate case Zhang and Shi (2016), the free surface acts as a deformable wall able to alter the vortex generation in the gap region. In figures 4.12 


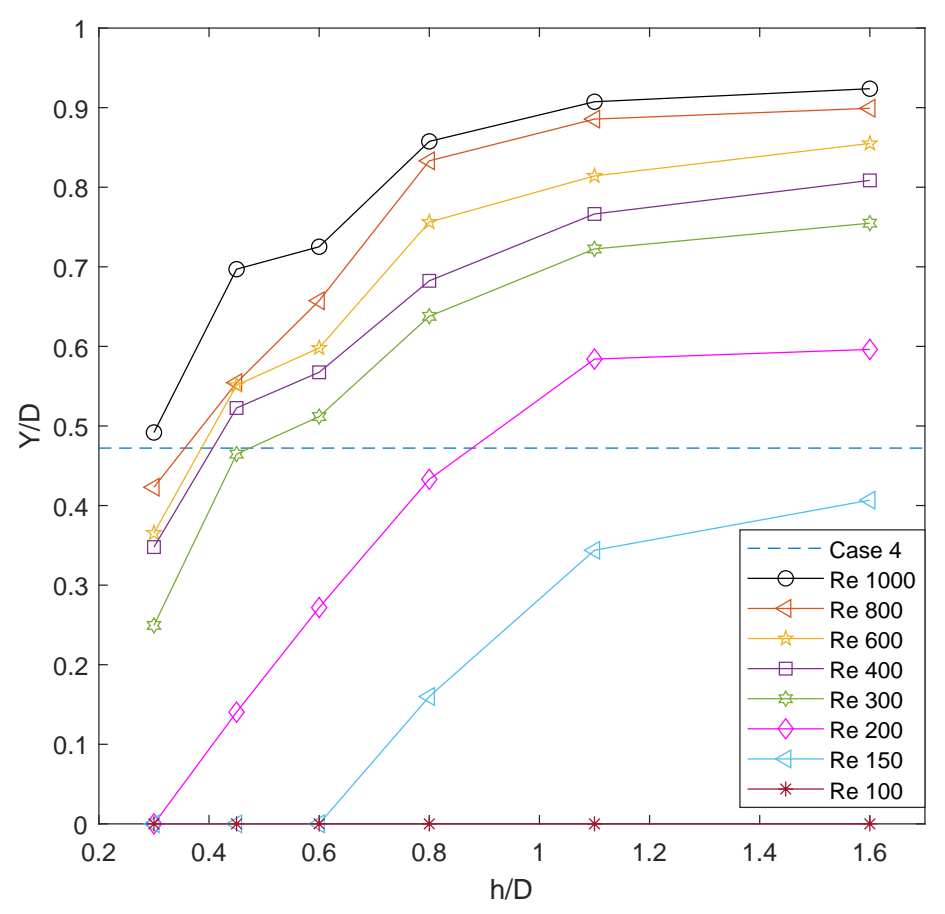

Figure 4.11: Vertical amplitudes of the plate tip versus depth for different Reynolds number values and different $h / D$ depths. The Froude $\mathrm{Fr}=2$ and the Cauchy numbers $C y=7.14 \cdot 10^{-4}$ are kept fixed. Case 4 , with uniform inflow velocity $U / \bar{U}=1$ and FSI3 properties (Table 4.3), has been included for comparison in a dashed blue line.

and 4.13, two dynamic pressure contour maps are shown for depths $h / D=0.8$ and $h / D=1.6$. In the plots, the pressure of the upper fluid is not coloured for an easier identification of the free surface. The pressure is presented in dimensionless form, with $p^{*}=\frac{p}{\rho \bar{U}^{2}}$. The proximity of the free surface modifies the pressure field around the structure and consequently affects the dynamic pressure acting on the plate. To have a clearer image of the dynamic pressure role on the plate deformation, the differences in dynamic pressure between both sides of the plate has been plotted for three different depths $h / D$ of $0.45,0.8$ and 1.6, when the tip of the plate is at its highest and at its lowest position, see figure 4.14. The cases with the larger depth imply larger dynamic pressure differences over the splitter plate hence, larger deformations. The free surface acts as a damper that prevents large oscillation amplitudes on the plate, dissipating part of the energy in wave formation.

Regarding the vorticity field, two runs consisting of 4 snapshots from a full oscillation cycle, are presented for two cases with different $h / D$, in figures 4.15 and 4.16 . The proximity of the free surface yields a reduction of the oscillatory character of the wake, and generates a flow deflection towards the opposite side of the free surface. When the structure is near the free surface, the lower shear layer seems not to reattach to the splitter plate. The wave generated on top of the structures, imposes a lower pressure region as seen in figures 4.12 and 4.13 , that forces a modification of the separation point on the upper surface of the cylinder. Under this situation, the upper shear layer is heavily conditioned by 


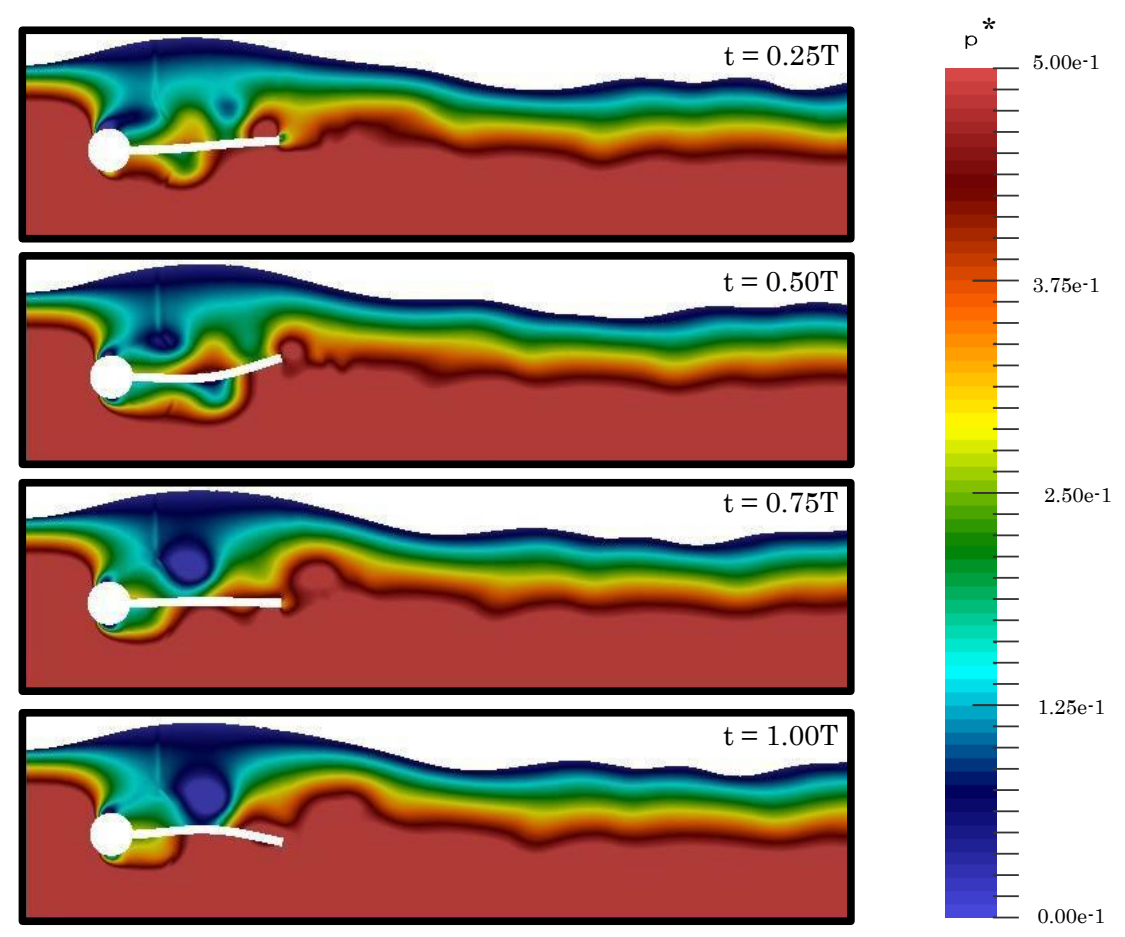

Figure 4.12: Snapshot of the normalized pressure field contour plot at $h / D=1.6$ during a complete shedding cycle of period $T$. The rest of parameters are: $\mathrm{Fr}=2, C y=7.14 \cdot 10^{-4}$ and $\operatorname{Re}=200$.

the wave shape and detaches from the cylinder at a considerably larger angle if compared to that in the lower part.

In order to understand better the effect of depth on the plate dynamics, a numerical experiment falling in the part of figure 4.11 in which the tip amplitude grows monotonically $(h / D=0.45$ and $R e=200$ ), therefore showing a large effect of the free surface, is analysed here. Selected points for the analysis are presented in figure 4.17 on top of the time series of the displacement of this particular case. In order to confirm the periodicity of the dynamic pressure field, points such as $P_{1}$ and $P_{9}$ are selected in the same part of the curve, but in a different oscillation cycle. The dimensionless dynamic pressure field $\left(p *=\frac{p}{\rho \bar{U}^{2}}\right)$ corresponding to each of the points described, appear in figure 4.18. The dynamic pressures at both sides of the splitter and the pressure difference are presented in figure 4.19.

The evolution of the dynamic pressure field represented in figure 4.18 shows that a low dynamic pressure region is formed at the top part of the structure. This low pressure region reminds a recirculation area that is consistently placed below the highest part of the quasi steady wave. The recirculation area changes its shape during the simulation, as can be seen in the first four snapshots of figure 4.18. Moreover, this is indicated by the fact that the dynamic pressure difference curves in figure 4.19, evolve as a wave travelling from left to right, and it is in snapshot P5 of figure 4.18, that a second pressure difference peak appears. The last is near the tip of the plate whilst the first is located at a position 

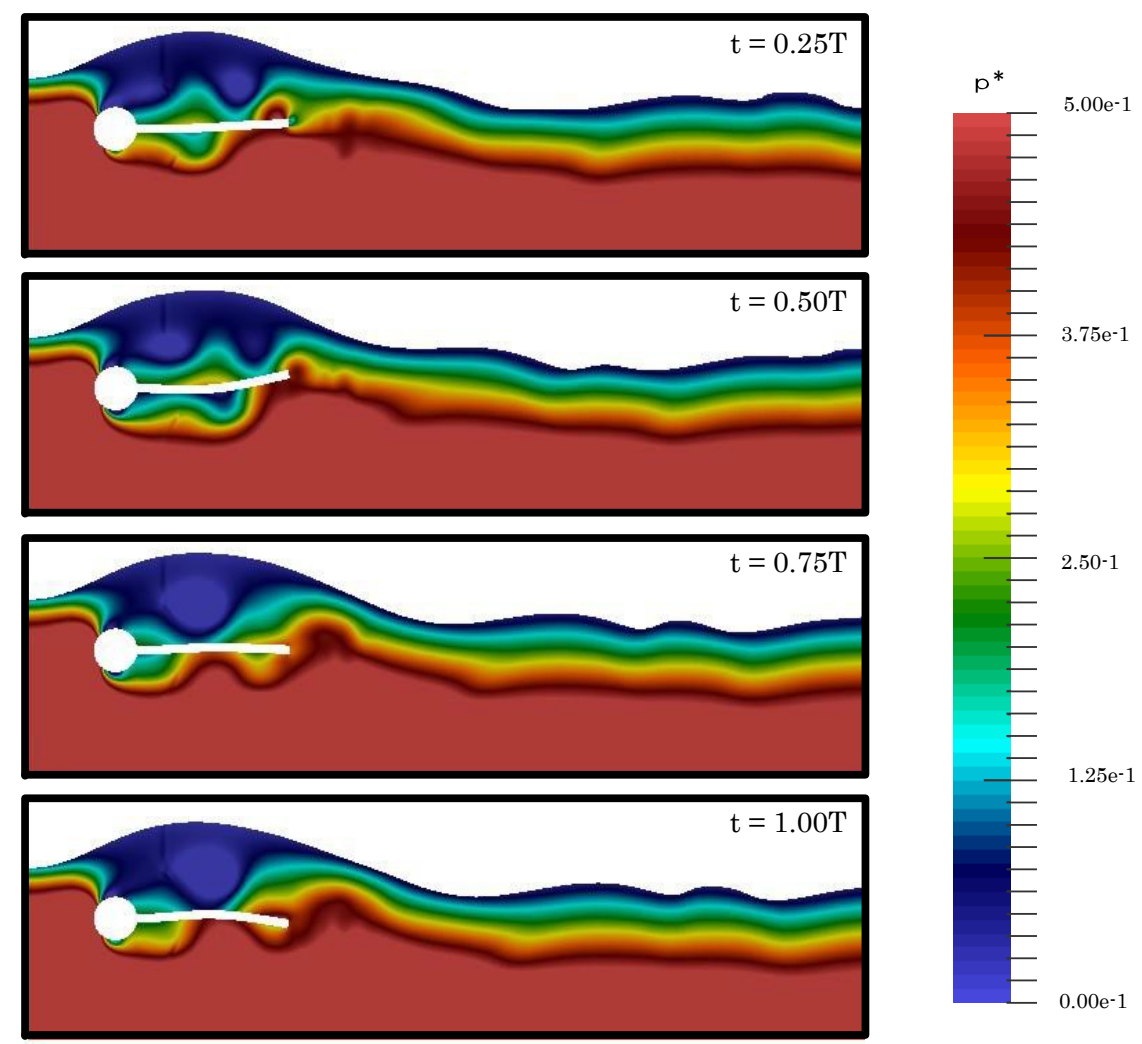

Figure 4.13: Snapshot of the normalized pressure field contour plot at $h / D=0.8$ during a complete shedding cycle of period $T$. The rest of parameters are: $F r=2, C y=7.14 \cdot 10^{-4}$ and $R e=200$.

$x / D \approx 1$. In snapshots $\mathrm{P} 5$ to $\mathrm{P} 8$ of figure 4.18 , these two pressure difference peaks move to the tip until one of them vanishes yielding a pressure different in snapshot $\mathrm{P} 9$, very similar to that in $\mathrm{P} 1$, with the cycle starting again.

\section{Additional comment about the absence of turbulence models}

As presented in Sumer and Fredsøe (1997) the wake behind a circular cylinder shows local turbulent features already at $R e>300$. Nonetheless, it is should be stressed that the geometry studied here is not an isolated cylinder, this geometry contains a long flexible plate on the wake area and also near walls which changes noticeably the flow and consequently the stability of the solution. This current geometry is more stable than the isolated cylinder, having most of the bifurcations and transitions delayed in terms of Reynolds number. In order to underline the differences caused by the plate, in 4.11 it can be observed that when $R e=100$, no deformation in the plate is observed, meaning that the flow is quite stable. At this Reynolds number, a periodic regime is obtained for the isolated cylinder case if there is no plate. Adding a long flat plate is a well-known mechanism to change the wake dynamics of a cylinder, consequently 


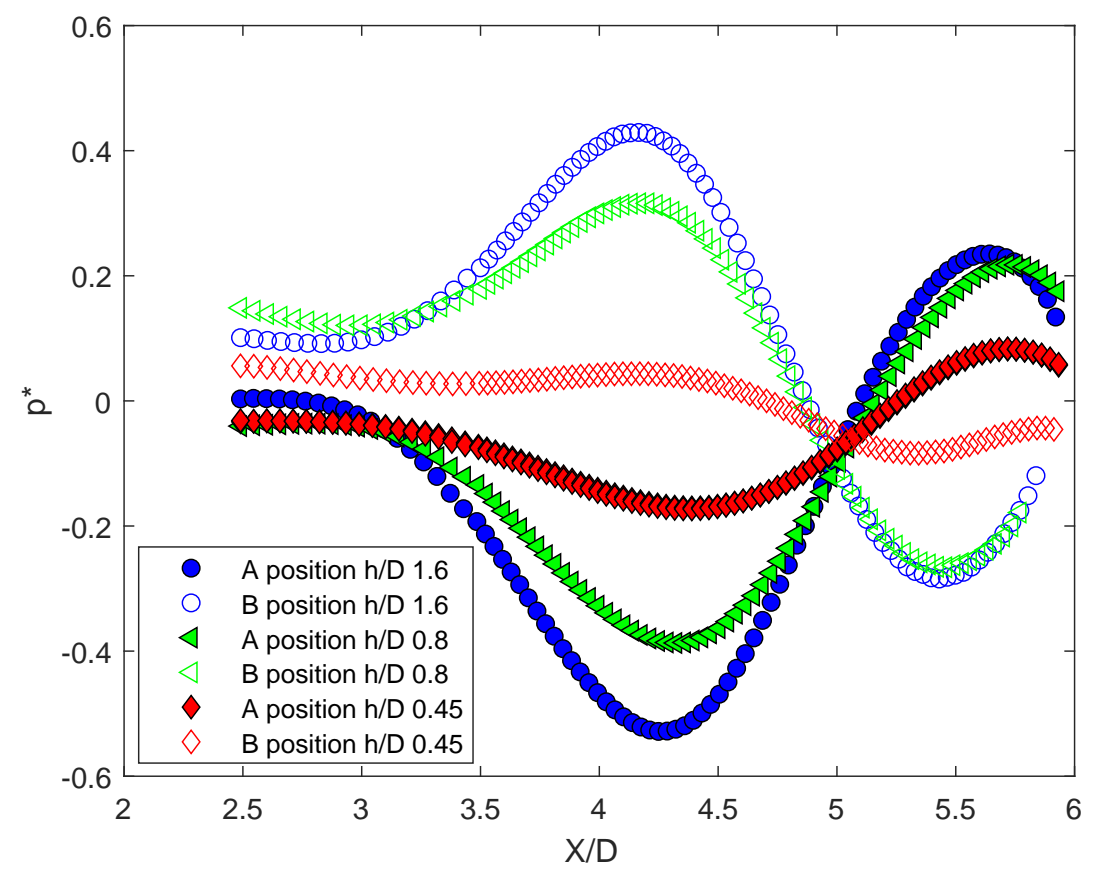

Figure 4.14: Pressure field difference along the plate for three different depths, when the tip of the plate is at the highest $(A)$ and lowest $(B)$ positions. The Froude $(F r=2)$ and the Cauchy numbers $(C y=7.14 e-4)$ are kept fixed.

a direct comparison with an isolated cylinder is not an adequate reference. In fact, the splitter plate suppresses the shear layer interaction mechanism in the near wake of the cylinder.

In Zdravkovich (1997) a highly detailed description about the flow around an isolated circular cylinder is presented. The author states that $\mathrm{Re}=1000$ can be considered as a transition shear layer state (TrSL). In this regime, 3 substates are considered, where $R e=1000$ could be classified into the first as TrSL1, as a typical case of development of transition waves. Which means that for that Reynolds number transition waves appear as undulations of the free shear layers a few diameters away from the cylinder, but this non-linear interaction is far from considering a turbulent flow which according to Zdravkovich (1997) appears between 20000 and 40000 (TrSL3). It is underlined again, that a plate of length 3.5D would increase this characteristic limit as it avoids the shear layers interaction. In addition, turbulence is a phenomenon characterized by the presence of an energy cascade and a characteristic frequency spectrum which is not the case of these flow at this limited Reynolds number since different test of the frequency spectrum in several points in the flow were performed, and always a discrete frequencies appear but never a typical turbulent spectrum.

In González (2001) the 2D isolated cylinder case obtained good global values such as $C_{D}, C_{L}$, but also local curves as the pressure profile around the cylinder perimeter and even normal velocity gradient on the cylinder surface for $\mathrm{Re}=1000$ when the mesh was well refined. As the geometry considered in 

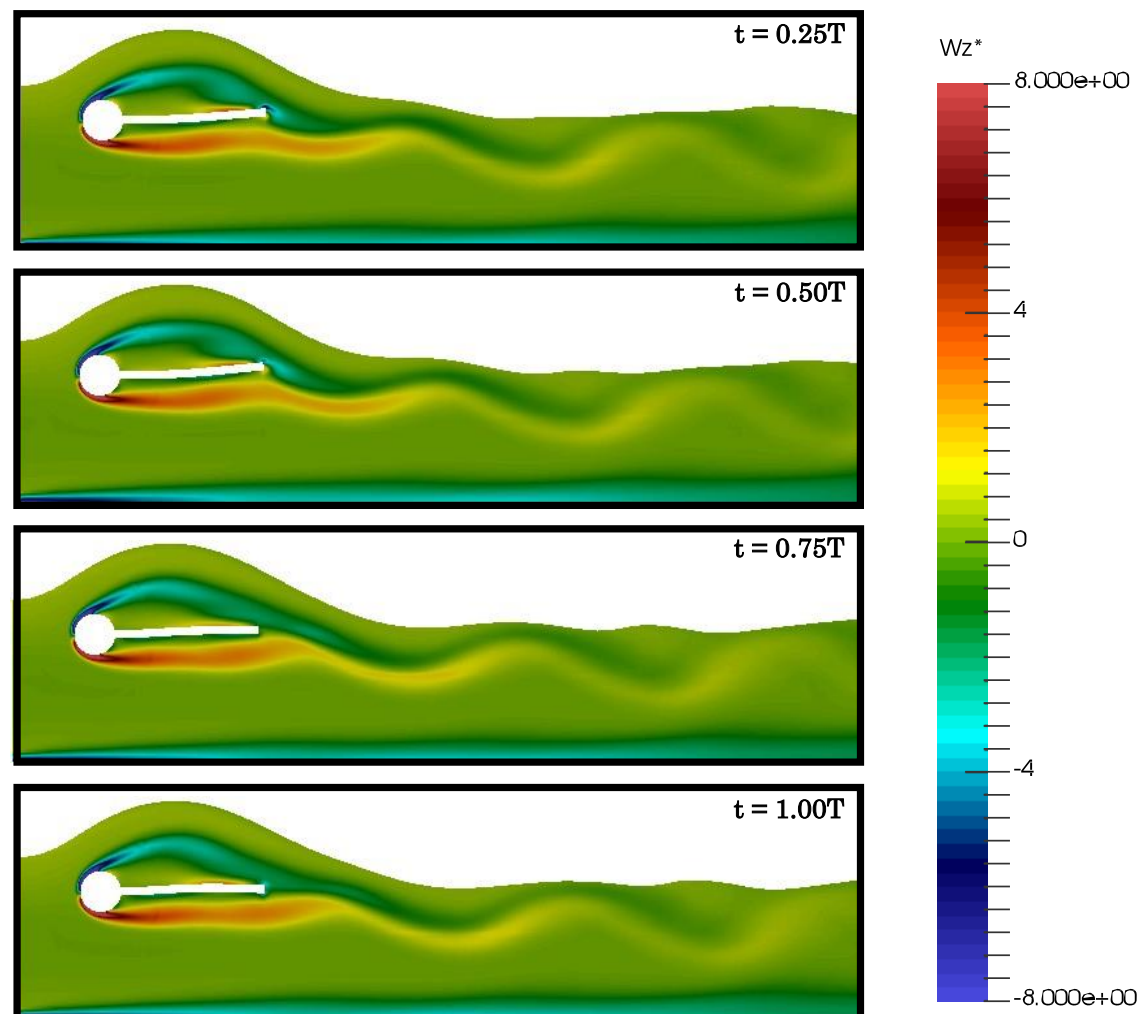

Figure 4.15: Snapshot of the vorticity field contour plot at $h / D=0.45$ during a complete shedding cycle of period $T$. Clockwise vorticity appears in blue (down to $\omega_{z_{m i n}}^{*}=-8$ ) and counter-clockwise in red (up to $\omega_{z_{\max }}^{*}=8$ ). The rest of parameters are: $\mathrm{Fr}=2, C y=7.14 \cdot 10^{-4}$ and $\operatorname{Re}=200$.

this problem is considerably more stable due to the long plate attached, the assumption of describing the flow without any turbulence model is still a very good approximation.

However, the possibility of considering the flow as $3 D$ for such Reynolds number that only means that the axial flow component has been neglected in particular for this fluid-structure interaction. Regarding the 3D, the question is how much affects the plate deformation and the FSI problem this threedimensionality. In De Nayer and Breuer (2014) performed at the limits included by Zdravkovich (1997) in the turbulent regime $R e=30000$ deformations of the plate are quasi two-dimensional. De Nayer and Breuer (2014) also shows that the transitions found at the shear layers which may develop high frequency dynamics, would be filtered out by the structure, being only the low frequency the ones directly visible in the structural part of the FSI phenomenon. For this work, only a future research about the 3D effect could properly quantify those effects.

\subsubsection{B Effect of Cauchy number}

In this subsection the dependence of the splitter plate dynamics on the Cauchy number is studied, see equation (3.22). Variations of this non-dimensional number have been achieved by altering the 

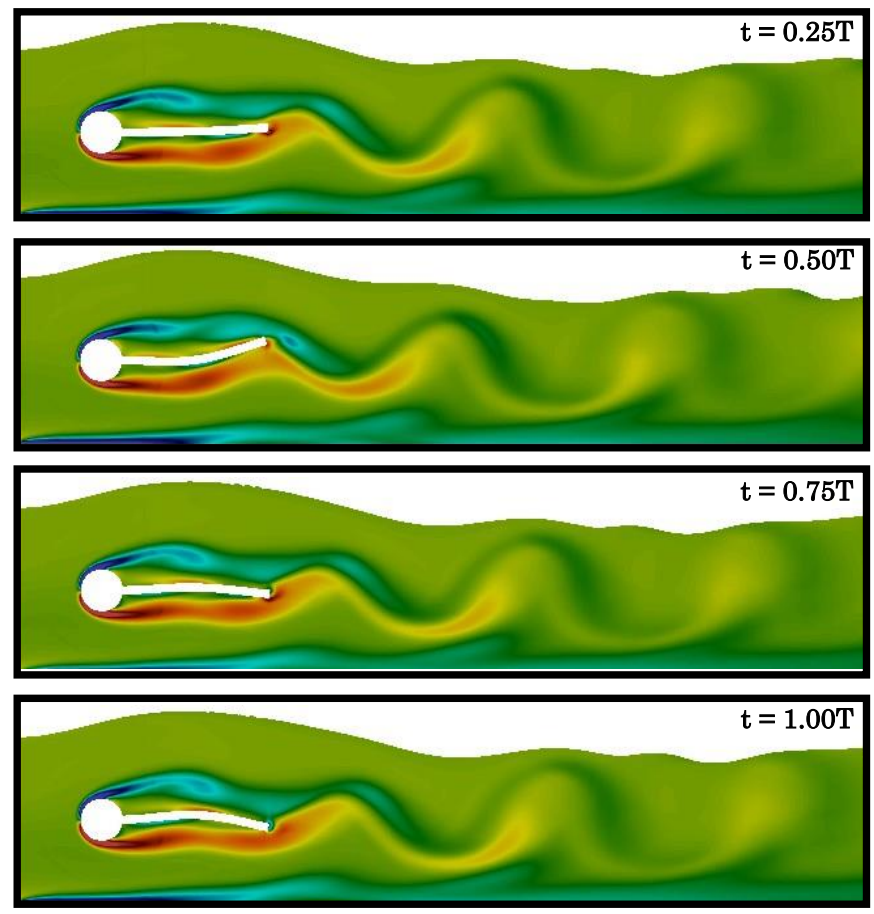

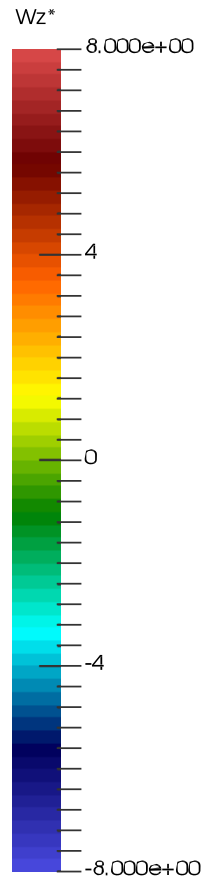

Figure 4.16: Snapshot of the vorticity field contour plot at $h / D=1.60$ during a complete shedding cycle of period $T$. Clockwise vorticity appears in blue (down to $\omega_{z_{m i n}}^{*}=-8$ ) and counter-clockwise in red (up to $\left.\omega_{z_{\text {max }}}^{*}=8\right)$. The rest of parameters are: $\mathrm{Fr}=2, C y=7.14 \cdot 10^{-4}$ and $\operatorname{Re}=200$.

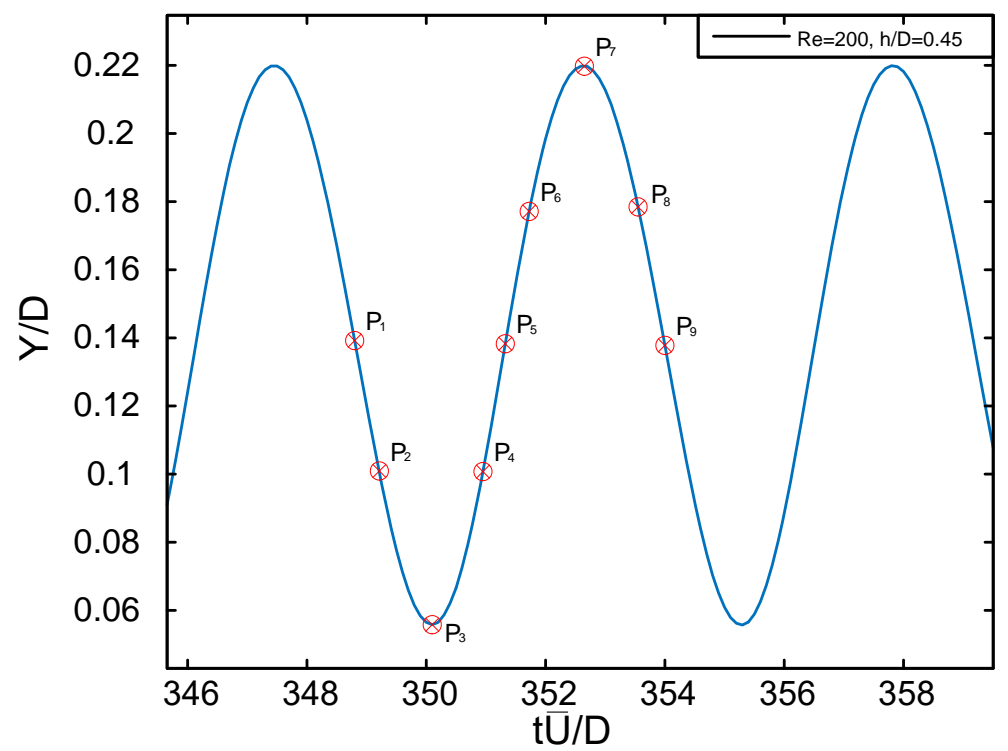

Figure 4.17: Vertical periodic displacement signal of point $A$ for $R e=200$ and $h / D=0.45$. Different positions $P_{i} \quad i=1, \ldots 9$ at different instants during one period have been selected for analysis.

Young's elastic modulus $E$ of the splitter plate, while keeping the Reynolds and Froude numbers constant with values of 200 and 2, respectively. The range of Cauchy numbers tested goes from $5.6 \cdot 10^{-5}$ to 

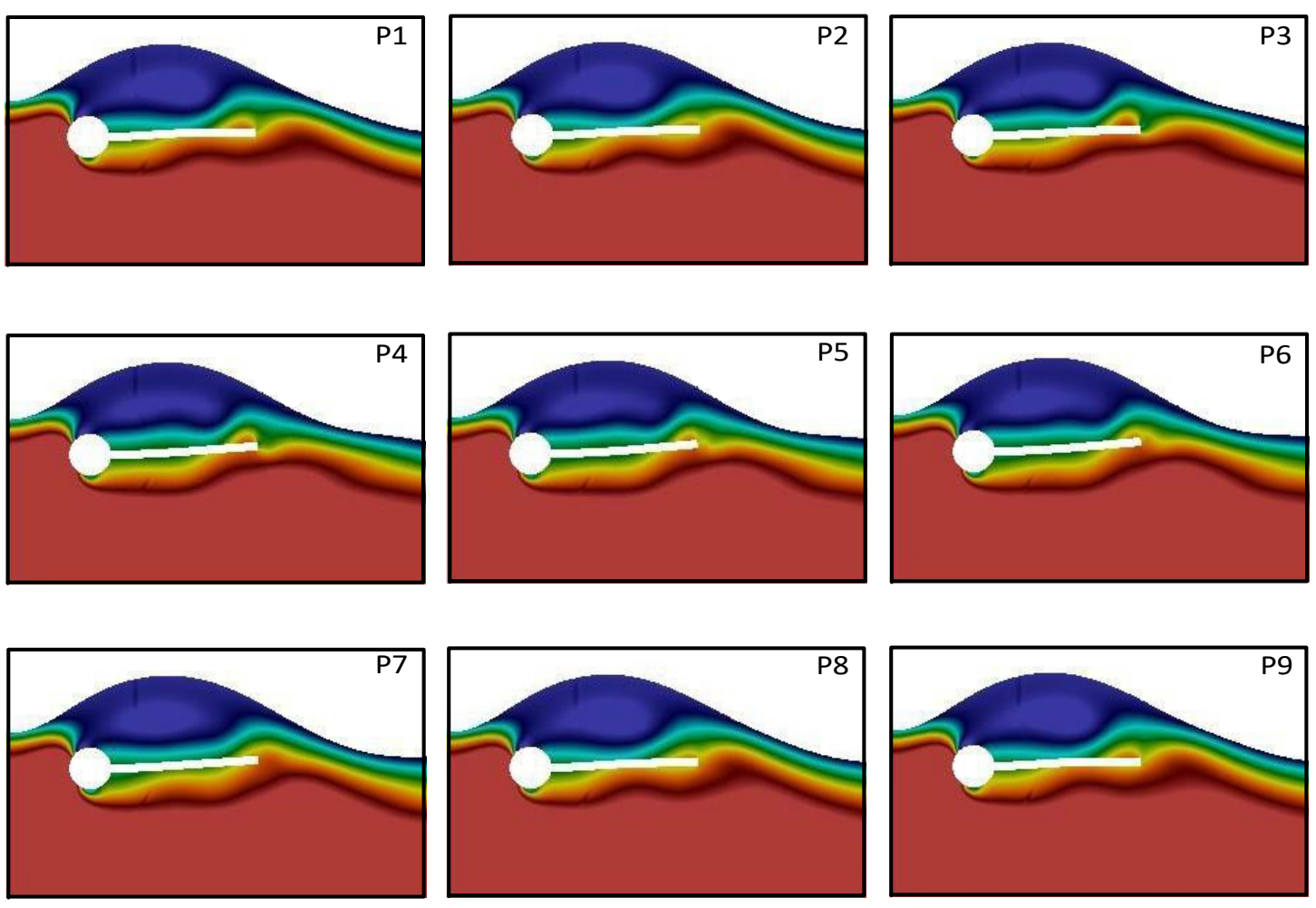

$0.00 \mathrm{e}^{-1}$

$1.25 \mathrm{e}^{-1}$

$p^{*}$

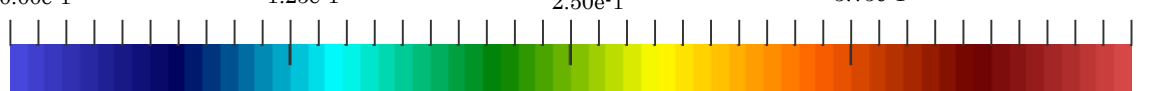

\section{$5.00 \mathrm{e}^{-1}$}

Figure 4.18: Contour normalized pressure field snapshots

$1.1 \cdot 10^{-3}$, including in this range the one used during the benchmark case with $C y=7.14 \cdot 10^{-4}$. Lower Cauchy numbers imply very little deformations, and higher Cauchy numbers make stable computations very difficult due to large mesh distortions.

The results obtained show that for a fixed $h / D$, if the stiffness of the plate is increased (higher Young's modulus and lower Cauchy number), the amplitude of the tip decreases, see figure 4.20. The plot includes the reference case used in figure 4.11 , showing a single fluid computation (case 4) with $C y=$ $7.14 \cdot 10^{-4}$, depicted with a dashed line. The same behaviour was also observed in the validation case FSI3 presented in section 4.3.1 Pisacreta (2017), with the amplitude decreasing as the Young modulus was increased. When higher stiffness is imposed to the plate, $C y \leq 5.26 \cdot 10^{-4}$, the system is unable to reach the amplitudes obtained without free surface for our range of depths. The oscillation amplitude of the point $A$ increases with $h / D$, and the vertical amplitude tends to an asymptotic value when the structure is moved away far from the free surface influence. We can observe here the same free surface damping. It is also worth noting that the plate remains stationary when depth is $h / D=0.3$ for all the 

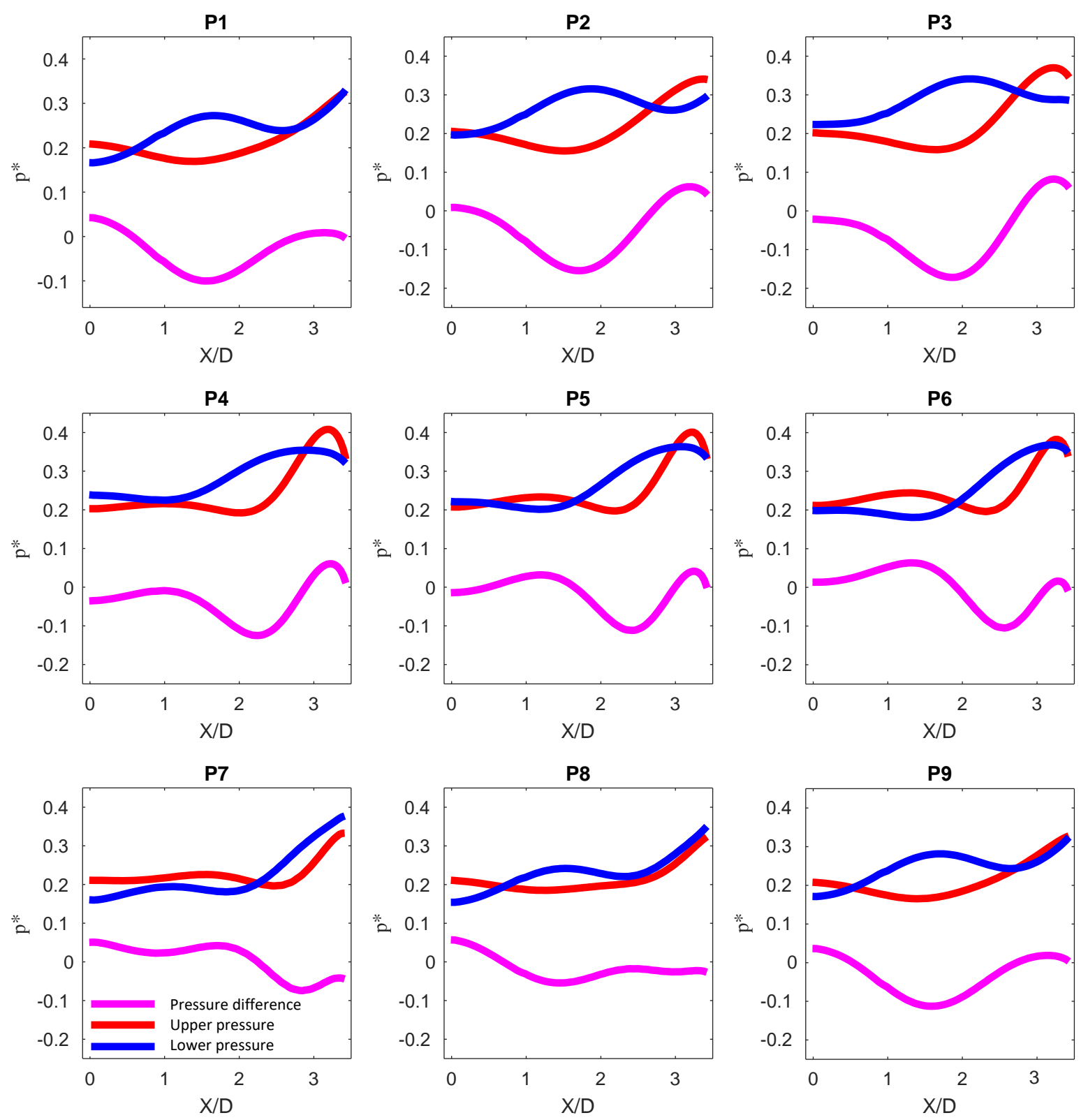

Figure 4.19: Normalized pressure values along the top, bottom of the structure and normalized pressure difference at the snapshot

stiffness tested. This is interpreted as the existence of a critical depth where the pressure difference between both sides is unable to break the stability of the plate for the range of Cauchy numbers tested. A linear dependence of the amplitude of the splitter plate with Cauchy number for the different depths, appears in figure 4.21 . 


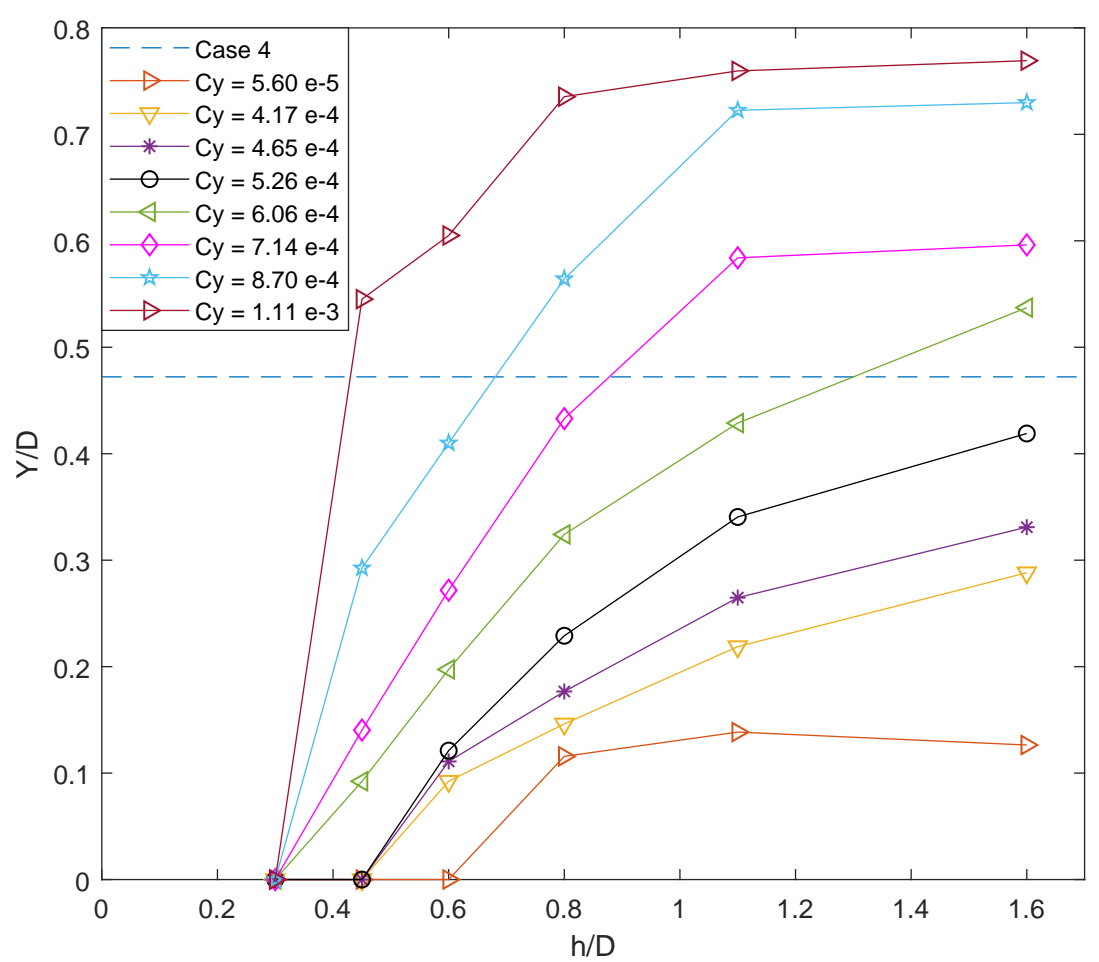

Figure 4.20: Splitter plate oscillation versus depth for different Cauchy numbers. The Froude and Reynolds numbers are constant with values: $F r=2$ and $R e=200$. Case 4, with uniform inflow velocity $U / \bar{U}=1$ and FSI3 properties (Table 4.3), has been included for comparison in a dashed blue line.

\subsubsection{Effect of Froude number}

In this case, the parameter varied in order to study the influence of the Froude number on the splitter plate dynamics, see equation 3.22, is the gravity acceleration. The rest of the non-dimensional numbers, such as Reynolds, mass and Cauchy numbers will be kept constant with values: $R e=200, C y=$ $7.14 \cdot 10^{-4}$ and $M=1$. The range of Froude numbers studied goes from 2 to 3.5 . The lower limit $F r=2$, corresponds to the realistic case where $g=9.8 \mathrm{~m} / \mathrm{s}^{2}$. Froude number higher than 3.5 were not examined due to the clear asymptotic behaviour of the plate oscillations found, see figure 4.22. Again reference case 4, with uniform inflow velocity $U / \bar{U}=1$ and FSI3 properties (Table 4.3), has been included using a dashed line, for comparison. From figure 4.22, it is clear how Froude number amplifies the splitter plate oscillations. For $F r \geq 2.5$, all the iso-Froude curves tend to converge to the same amplitude level, meaning that at this stage, there is very little influence of the Froude number on the plate dynamics, therefore inertial terms are more important than gravitational ones. For the smallest depth tested, $h / D=0.3$, the data suggests that there is a critical Froude number in the interval $[2,2.5]$ if any plates motions are to be observed. The absence of a free surface decreases the vertical oscillation amplitude when compared to the gravitational cases for $F r \geq 2.5$.

In this section, the fluid forces acting on the whole structure, including the cylinder and the splitter 


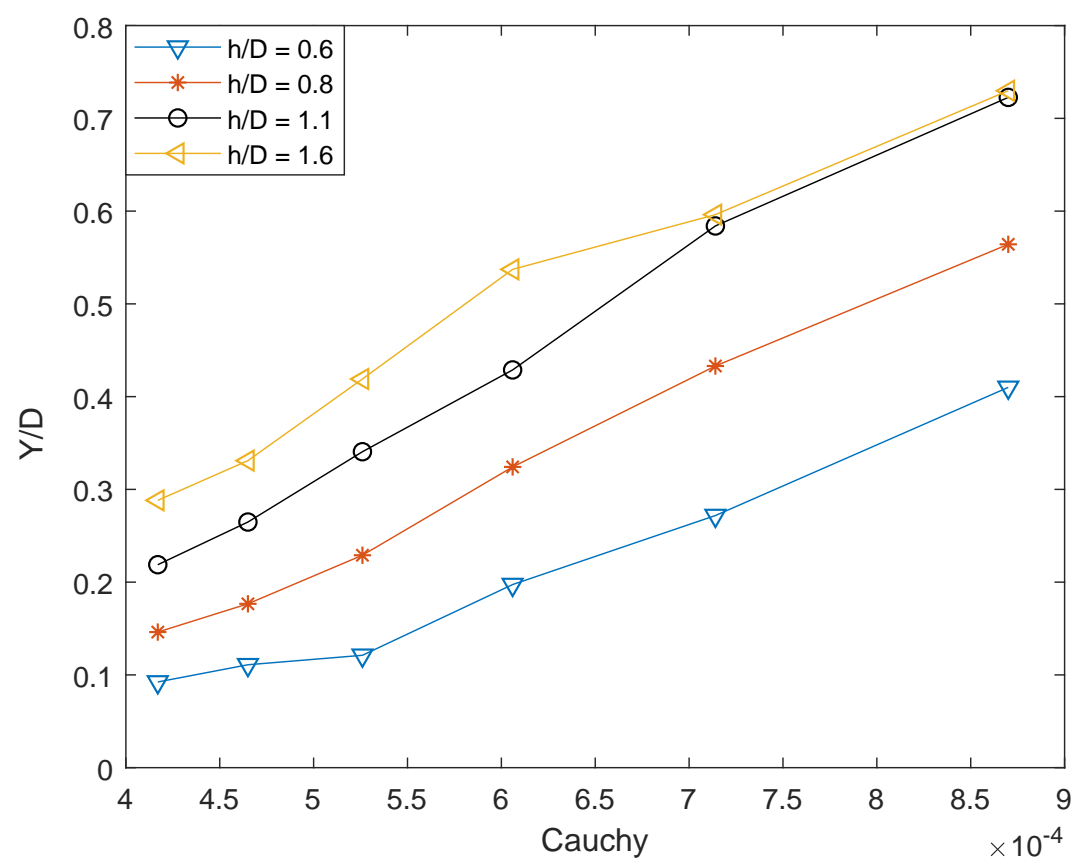

Figure 4.21: Comparison between the numerical simulations by changing the Cauchy number and keeping the Froude $\mathrm{Fr}=2$ and the Reynolds $R e=200$ numbers constant.

plate, are studied by varying the dimensionless numbers in the same way as in the previous section. Both, pressure and viscous forces will be integrated over the structure surface, and projected on the horizontal axis to obtain the drag force $F_{x}$. In order to compare different cases, and taking into account the oscillatory nature of these forces, a time average of the force components is calculated once a quasi periodic oscillation regime is obtained. These forces will be non-dimensionalized using equation 4.2.

$$
C_{D}=\frac{F_{x}}{\frac{1}{2} \rho_{f} \bar{U}^{2} c D}
$$

\subsubsection{Effect of Reynolds number}

Similarly to what was performed in section 4.3.3, several simulations were carried out changing the Reynolds number value $R e \in[100,1000]$ in order to understand the influence of this parameter on the plate forces. The other non dimensional numbers were kept constant as $C y=7.14 \cdot 10^{-4}$ and $F r=2$ during the simulations.

A dashed line is included in Figure 4.23, reference case 4, with uniform inflow velocity $U / \bar{U}=1$ and FSI3 properties (Table 4.3), to allow comparisons. A reduction of the horizontal force $F_{x}$ takes place when the Reynolds number is increased in this laminar range as a consequence of a decrease of the viscous component of the force. This trend is similar to that of the drag coefficient of an isolated cylinder 


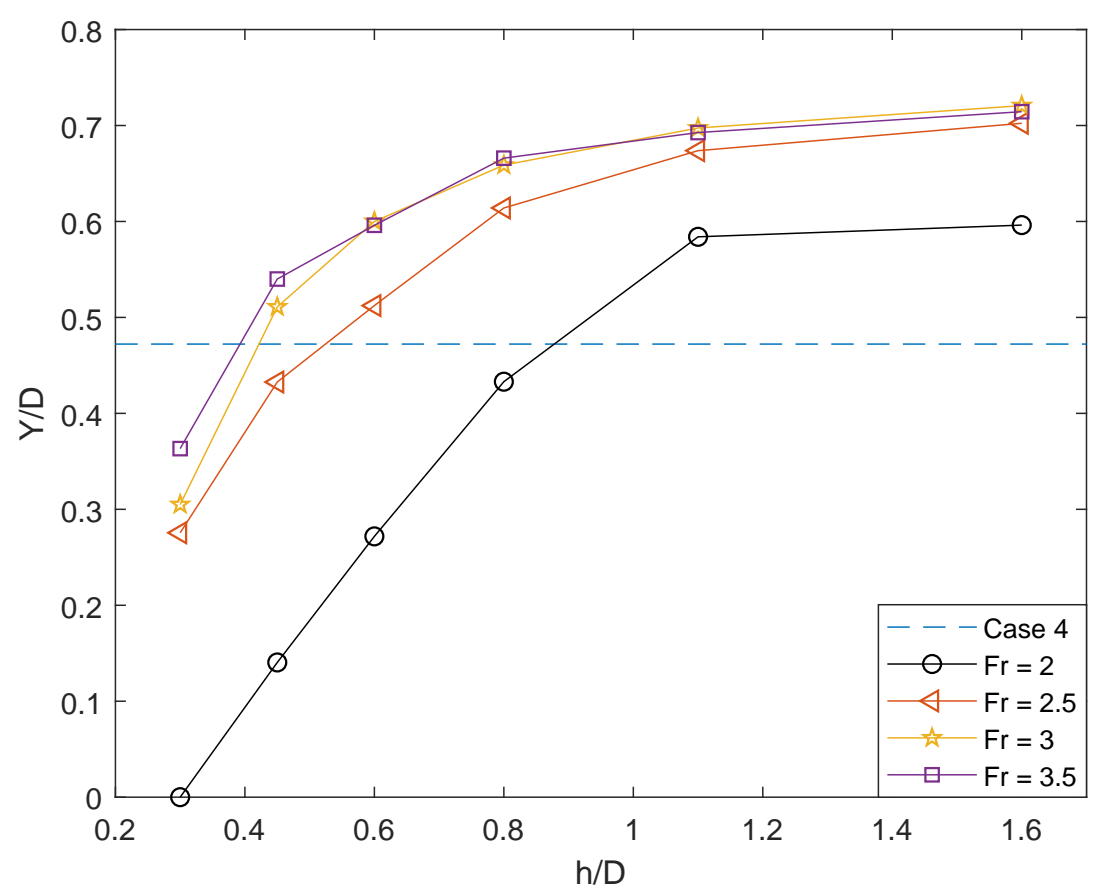

Figure 4.22: Vertical oscillation amplitude of point $A$ by changing the Froude number and keeping constant the Cauchy number $C y=7.14 \cdot 10^{-4}$ and the Reynolds number $R e=200$. Case 4, with uniform inflow velocity $U / \bar{U}=1$ and FSI3 properties (Table 4.3), has been included for comparison in a dashed blue line.

for Reynolds numbers increasing in the same range. In terms of depth, the drag value increases linearly for all Reynolds numbers. This was previously reported by Bouscasse et al. (2017), with a Froude number of 2, when the authors studied the case of an isolated cylinder near the free surface. Moreover, if we compare the result for $R e=200$ and the largest depth $h / D=1.6$ to the single fluid case 4 , we observe that the drag result is barely increased. When the parameter $h / D$ is lower and the structure is closer to the free surface, the drag force coefficients do not match due to the free surface influence.

\subsubsection{E Effect of Cauchy number}

The range of Cauchy numbers studied here is the same as in section 4.3.3, with the main results presented in figure 4.24. The reference case 4, with uniform inflow velocity $U / \bar{U}=1$ and FSI3 properties (Table 4.3), has been included again for comparison. Focusing on the free surface cases, as all curves for constant Cauchy numbers tend to merge, the dependence on the Cauchy number barely affects the averaged drag. Consequently, the strong dependence of oscillation amplitude which clearly increases when the Cauchy number is reduced, see figure 4.20 , is not followed by the averaged drag force. In the figure it is obvious how drag values increase linearly with depth independently of the bending stiffness of the plate. This is an indication of the drag term being dominated by the cylinder instead of by the dynamics of the plate. As in the previous results with the $R e$, the time averaged drag forces tend to the 


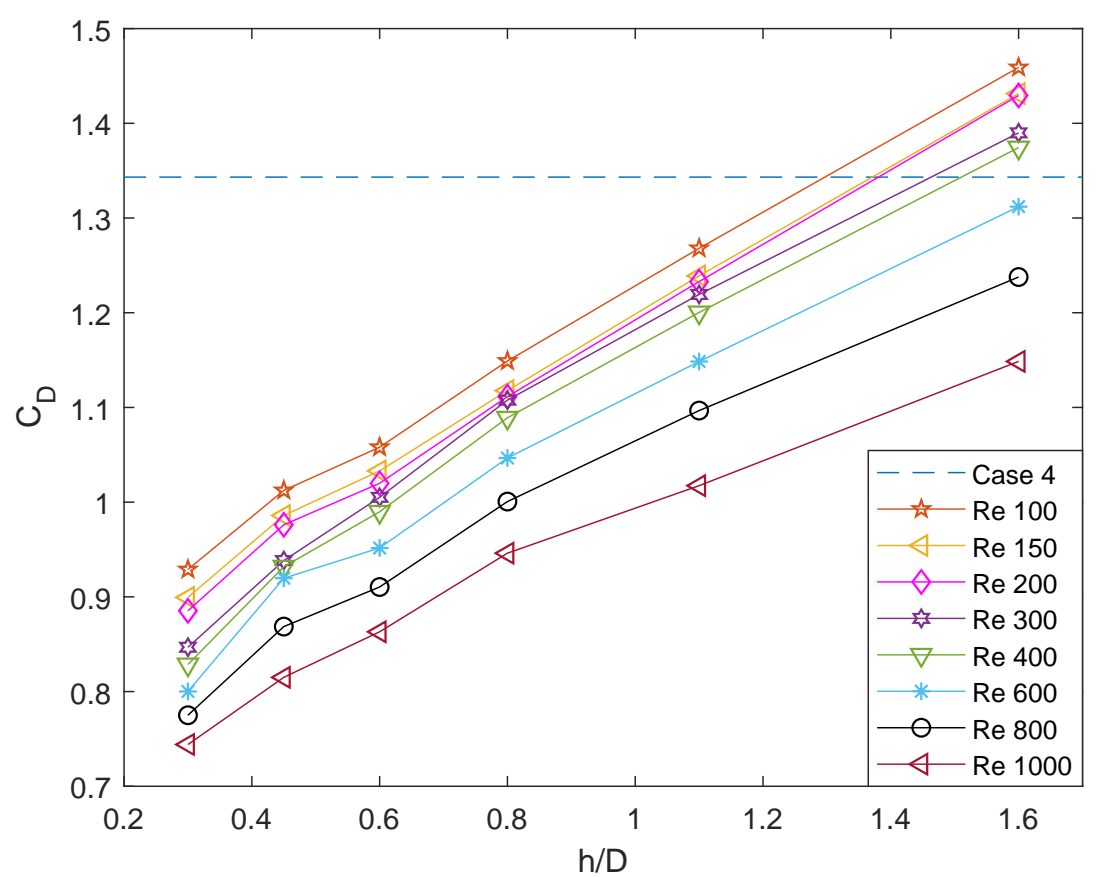

Figure 4.23: Drag coefficient versus depth for different Reynolds numbers while keeping constant the Froude $F r=$ 2 and Cauchy numbers $C y=7.14 \cdot 10^{-4}$. Case 4 , with uniform inflow velocity $U / \bar{U}=1$ and FSI3 properties (Table 4.3), has been included for comparison in a dashed blue line.

value obtained in reference case 4 , as the depth is increased.

\subsubsection{F Effect of Froude number}

Here, we analyse how the drag forces vary with depth and with Froude numbers, keeping constant $R e=200$ and $C y=7.14 \cdot 10^{-4}$. Results appear in figure 4.25, where a small dependence of the drag force on the Froude number is depicted. Curves corresponding to the different Froude numbers are close to each other, especially at large depths. The dashed line represents case 4 , with uniform inflow velocity $U / \bar{U}=1$ and FSI3 properties (Table 4.3), has been included for comparison. Drag increases almost linearly with depth for all Froude numbers. Although the Froude number range is very different to that in Bouscasse et al. (2017), the results presented here for $(\operatorname{Re}, F r, h / D)=(200,2,0.6)$, are comparable as they showed a value of 0.92 with $(R e, F r, h / D)=(180,2,0.55)$. Again, only at the largest depths, with a mild effect of the free surface, results tend to the ones obtained in reference case 4 .

\subsubsection{Transverse force frequency in the presence of the free surface}

In order to evaluate whether the excitation frequencies associated to the transverse force component are close to the natural frequencies of the splitter plate, they have been computed analytically and expressed in dimensionless form using the term $D / \bar{U}$. We assume the natural frequencies of the splitter 


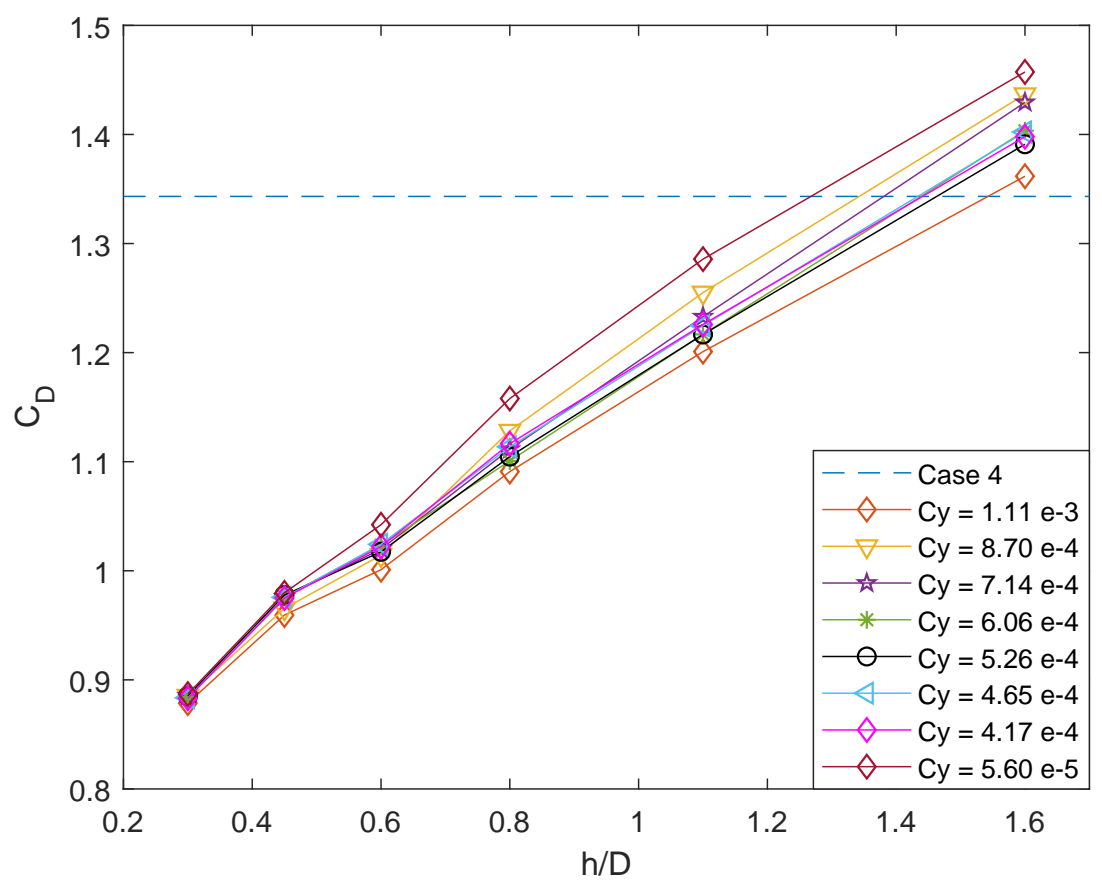

Figure 4.24: Averaged drag coefficients $C_{D}$ changing the Cauchy number and keeping constant the Froude $F r=2$ and the Reynolds $R e=200$ numbers. Case 4 , with uniform inflow velocity $U / \bar{U}=1$ and FSI3 properties (Table 4.3), has been included for comparison in a dashed blue line.

plate can be computed using a model for a cantilevered beam, as given by equation Kundu et al. (2017).

$$
f_{n_{i}}^{*}=\frac{D}{\bar{U}} \frac{k_{i}^{2}}{2 \pi} \sqrt{\frac{E I}{\rho_{s} A l^{4}}}
$$

where $E I$ is the chord-wise flexural stiffness and $A$ is the cross-sectional area. $\rho_{s}$ is used for the material density and $k_{i}$ is a constant that is dependent on the mode number $i$, and for the first three modes has values of $1.875,4.694$ and 7.855 . The natural frequencies of the first three modes of vibration, estimated using the above equation, appear in table 4.6.

Figure 4.26 depicts a situation in which with the exception of the cases with the lowest $R e$ and when close to the free surface, all runs had monotonically increasing frequencies around 0.2. The same happens in figure 4.27 , but in this case for the highest Cauchy numbers. Moreover, the effect of Froude number is very limited as can be seen in figure 4.28. The results indicate how the phenomena is not based on resonance, as natural frequencies are away from the responding frequencies and therefore oscillations are caused by the flow field fluctuations around the splitter plate. Compared with the Strouhal number of an isolated cylinder $S t=0.2$ for the range of $R e\left[10^{2}, 10^{3}\right]$. The trends imposed by the varying depth yield a different behaviour if compared to that in the results by Zhang and Shi (2016), Rao et al. (2013) and Huang and Sung (2007) in which a fixed solid wall instead of a free surface acted as the 


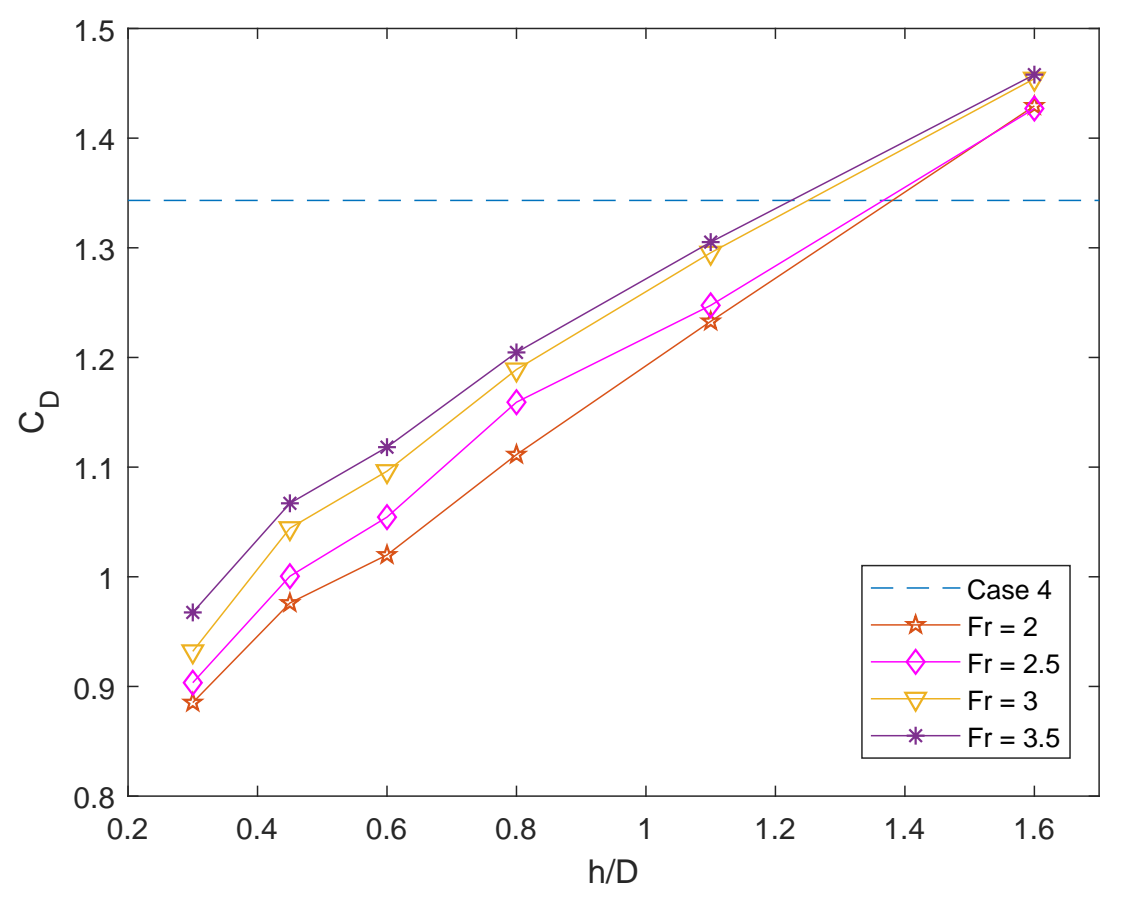

Figure 4.25: Averaged drag coefficient versus depth for different Froude numbers, while keeping constant the Cauchy $C y=7.14 \cdot 10^{-4}$ and the Reynolds $R e=200$ numbers. Case 4, with uniform inflow velocity $U / \bar{U}=1$ and FSI3 properties (Table 4.3), has been included for comparison in a dashed blue line.

\begin{tabular}{cccc}
\hline Cy & $f_{n_{1}}^{*}$ & $f_{n_{2}}^{*}$ & $f_{n_{3}}^{*}$ \\
\hline $1.11 \mathrm{e}-3$ & 0.11 & 0.070 & 0.196 \\
$8.70 \mathrm{e}-4$ & 0.013 & 0.079 & 0.222 \\
$7.14 \mathrm{e}-4$ & 0.014 & 0.087 & 0.245 \\
& & & \\
$6.06 \mathrm{e}-4$ & 0.015 & 0.094 & 0.266 \\
& & & \\
$5.26 \mathrm{e}-4$ & 0.016 & 0.102 & 0.285 \\
$4.65 \mathrm{e}-4$ & 0.017 & 0.108 & 0.303 \\
$4.17 \mathrm{e}-4$ & 0.018 & 0.114 & 0.321 \\
$7.15 \mathrm{e}-5$ & 0.044 & 0.277 & 0.774 \\
\hline
\end{tabular}

Table 4.6: Natural frequencies numbers computed analytically for the different Cauchy numbers. 


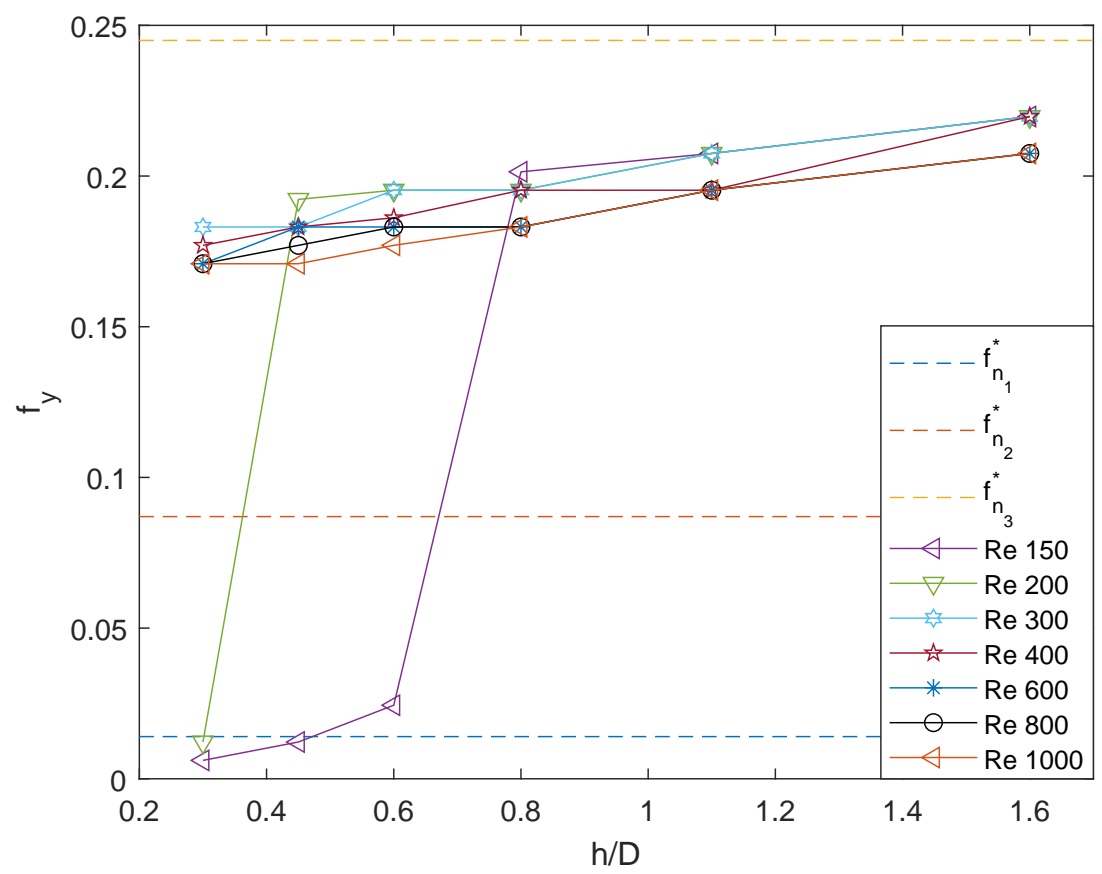

Figure 4.26: Frequencies versus depth for different Reynolds numbers, while keeping constant the Froude $F r=2$ and the Cauchy $C y=7.14 \cdot 10^{-4}$ numbers.

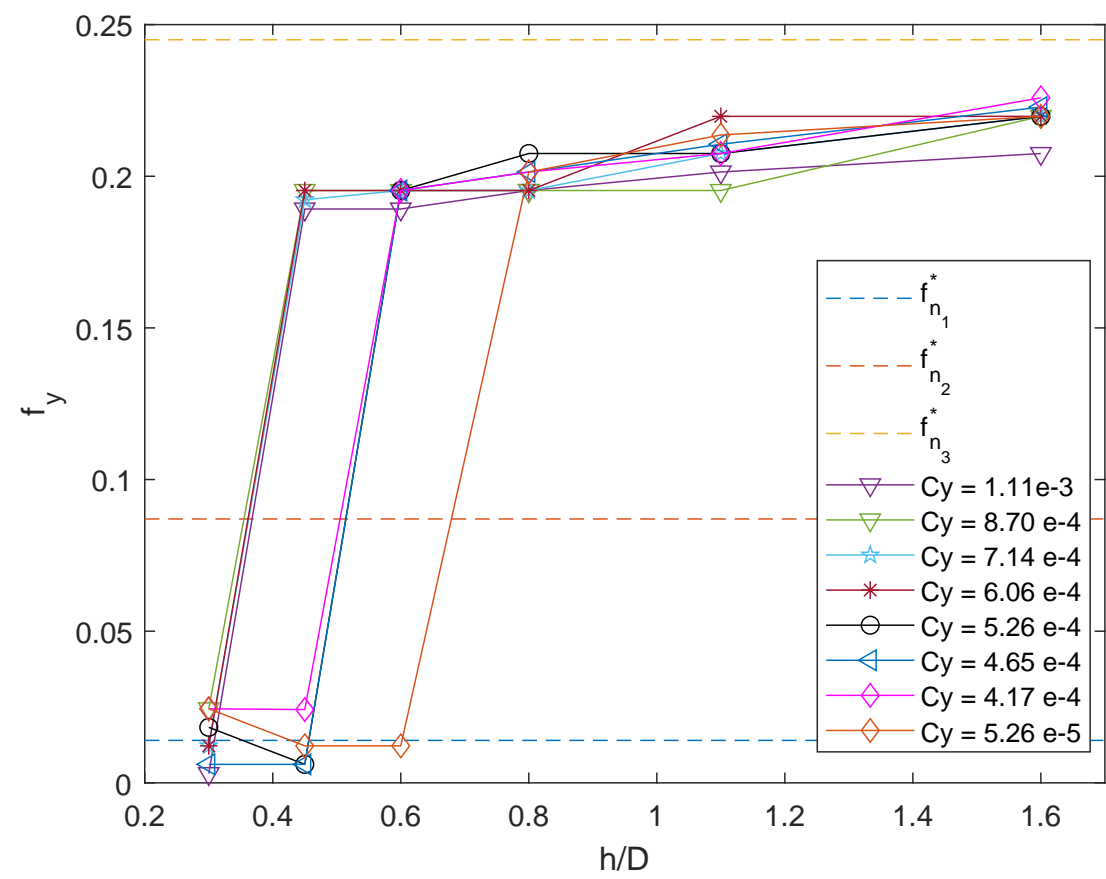

Figure 4.27: Frequencies versus depth for different Cauchy numbers, while keeping constant the Froude $\mathrm{Fr}=2$ and the Reynolds $R e=200$ numbers. 


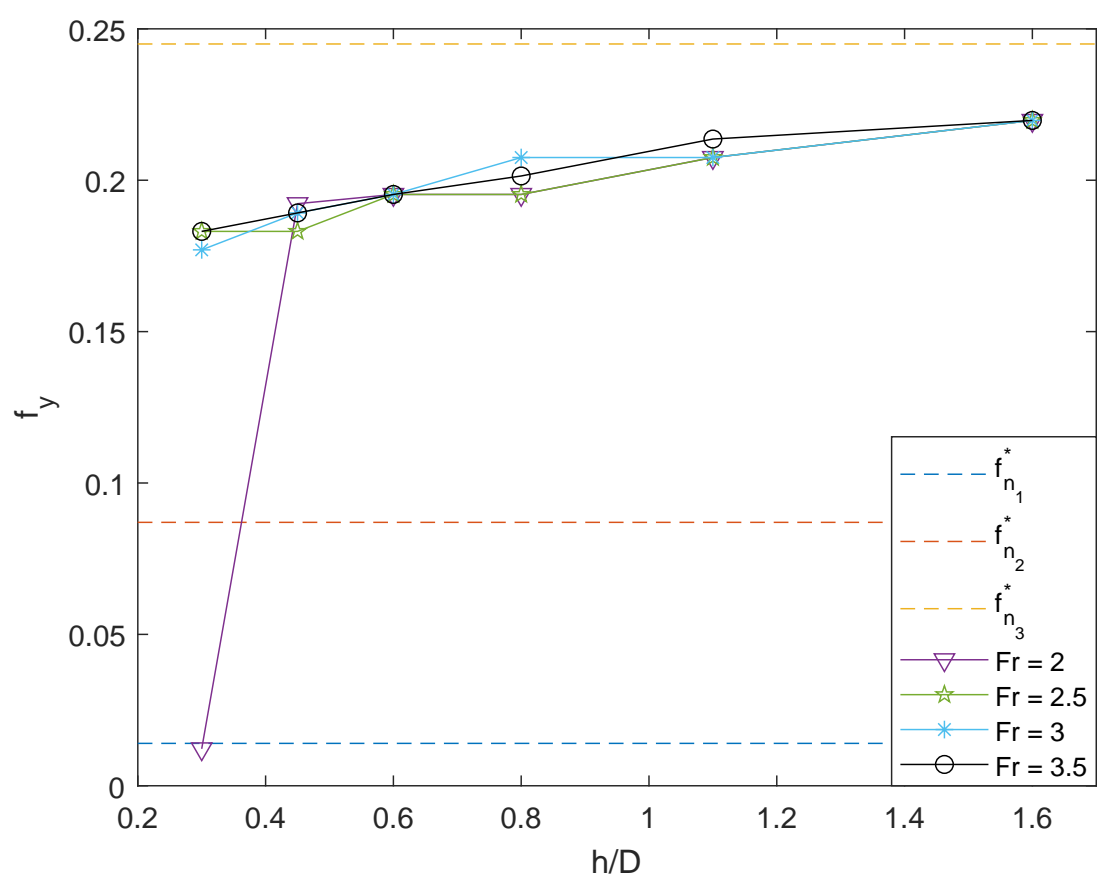

Figure 4.28: Frequencies versus depth for different Froude numbers, while keeping constant the Cauchy $C y=$ $7.14 \cdot 10^{-4}$ and the Reynolds $R e=200$ numbers.

near boundary, in studies conducted with rigid plates. In contrast to the rigid plate results presented by Zhang and Shi (2016), here there is no abrupt decrease of frequency for Reynolds numbers higher than 300 , and the behaviour found for all Reynolds numbers is very similar.

\subsection{Chapter conclusions}

A two dimensional analysis of the dynamics of a flexible splitter plate attached to the base of a cylinder, in laminar the regime has been carried out, using numerical techniques. The study is focused on the understanding of the effects of the free surface and the gravity field on the dynamic response and excitation of the elastic splitter plate. The numerical domain is divided by the free surface in two regions with different immiscible fluids. The structure consists of a stationary rigid cylinder that has an elastic splitter attached to its base. The work is inspired by the benchmark case posed by Turek et al. (2010), but adding the free surface effects. Reynolds, Froude and Cauchy numbers are systematically varied together with the distance from the structure to the free surface, covering a large parametric space.

Results show how the response of the splitter plate grows monotonically with increasing depth up to a saturated condition in which the free surface does not play a role any more. Moreover, the amplitude of the tip of the splitter plate increases with the Reynolds, Froude and Cauchy numbers as well. The free surface acts as a damping source for the plate dynamics reducing its oscillations. The wave that 
appears on top of the structure moves forward the separation point of the boundary layer in the upper surface of the cylinder, generating an asymmetry in the shear layers as they detach from the cylinder, yielding a pressure difference that results in decreased splitter oscillations. Within our parameter range, the splitter plate deflects with a shape that is based on either a first mode of vibration or a combination of the first and the second modes. The dominant frequencies of the oscillations are far from the natural frequencies estimated for the splitter plate, suggesting non resonant conditions. The drag coefficient increases linearly with depth, and decreases as expected when the viscosity of the fluid is reduced, without presenting a relevant dependence on the Cauchy and Froude numbers. 
CHAPTER 4. LAMINAR SIMULATION OF THE EFFECT OF THE FREE SURFACE ON A FLEXIBLE SPLITTER PLATE IN THE WAKE OF A STATIONARY CYLINDER 


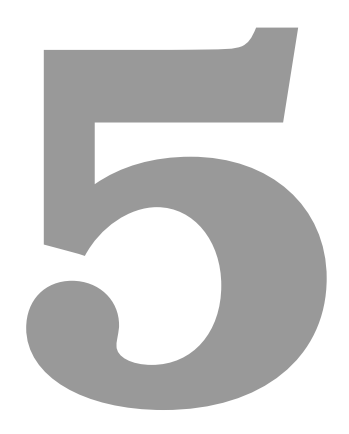

\section{Large Eddy Simulation of the flow past}

\section{a normal thin plate with free surface}

\section{Contents}

5.1 Introduction $\ldots \ldots \ldots \ldots \ldots \ldots \ldots \ldots \ldots \ldots \ldots \ldots \ldots \ldots \ldots \ldots$

5.2 Problem description. . . . . . . . . . . . . . . . . . 54

5.3 Results: Validation of the numerical set-up . . . . . . . . 56

5.4 Results: Full 3D simulations involving free surface $\ldots \ldots \ldots \ldots 1$

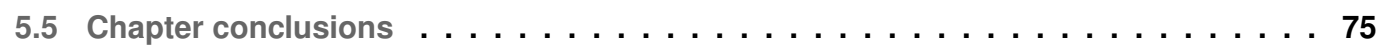




\subsection{Introduction}

In this work, we study the problem of the hydrodynamics of a flat plate normal to the flow in the turbulent regime, considering the effect of the free surface. This is in fact, a poorly understood canonical problem with great industrial implications, that has not received the deserved attention, neither experimentally nor numerically. This chapter is organised as follows: First, the problem description is presented in 5.2 where the mesh details and the main parameters are introduced. In Sec. 5.3 the methodology is validated for the 3D-periodic case as in Tian et al. (2014). The effects of the free surface on the drag and lift forces, on the pressure field and on different hydrodynamic aspects are discussed. Finally, concluding remarks are presented in section 5.5.

\subsection{Problem description}

The dimensions of the computational domain, depicted in figure 5.1, are $K, G$ and $J$ in the streamwise $(x)$, chordwise $(y)$ and spanwise $(z)$ directions, respectively. The cross-section of the plate has a height $b$ (y-axis) and a width $c$ ( $z$-axis) with a thickness $t \ll b, c$. The centre of coordinates is located at the centre of the plate. We have studied two different cases, a 3D-periodic case with a single phase and a pure 3D normal flat plate. The 3D case is studied using two different configurations with a single phase flow (no free-surface but a rigid wall) and a two phase flow (free surface). The results obtained with the last configurations are compared with recent experiments Satheesh and Huera-Huarte (2019). The heigth of the air phase if present, is $R>5 \mathrm{~cm}$. When two immiscible Newtonian fluids, air and water, are involved in the problem, gravity and free surface are obviously a part of the problem.
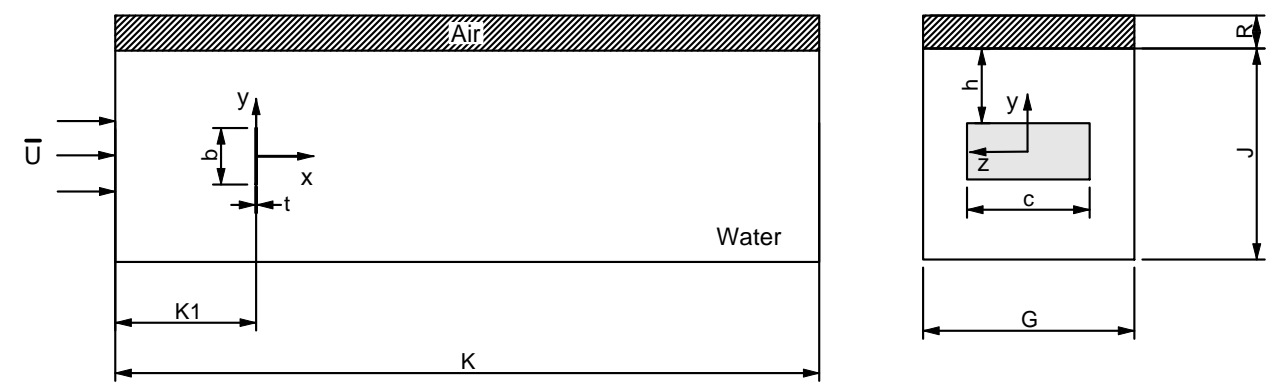

Figure 5.1: Schematic of the computational domain. The air phase is only present in the case that involves free surface simulations, not in the case with the rigid wall

Different meshes were studied in order to have an accurate computation of the different flow fields produced, and converged measurements of the forces acting on the plate. The mesh structure is always the same as depicted in figure 5.2, with the mesh size and consequently the number of cells varied 
to obtain the different meshes used. The number of cells on each side depends on the value a single mesh parameter which increases the number of cells. The mesh strategy used for the 3D-periodic case is the same as in the 3D case with the addition of the air phase in the upper domain. Notwithstanding the mesh convergence study, a time step study was also performed. The conclusion is that a time step $\Delta t>1 \times 10^{-3}$ induces numerical instabilities that lead failures in the simulation. The time step selected for all the simulations is $\Delta t=5 \times 10^{-3}$, leading to errors that are similar to those obtained with smaller time steps, but less expensive from a computational point of view.
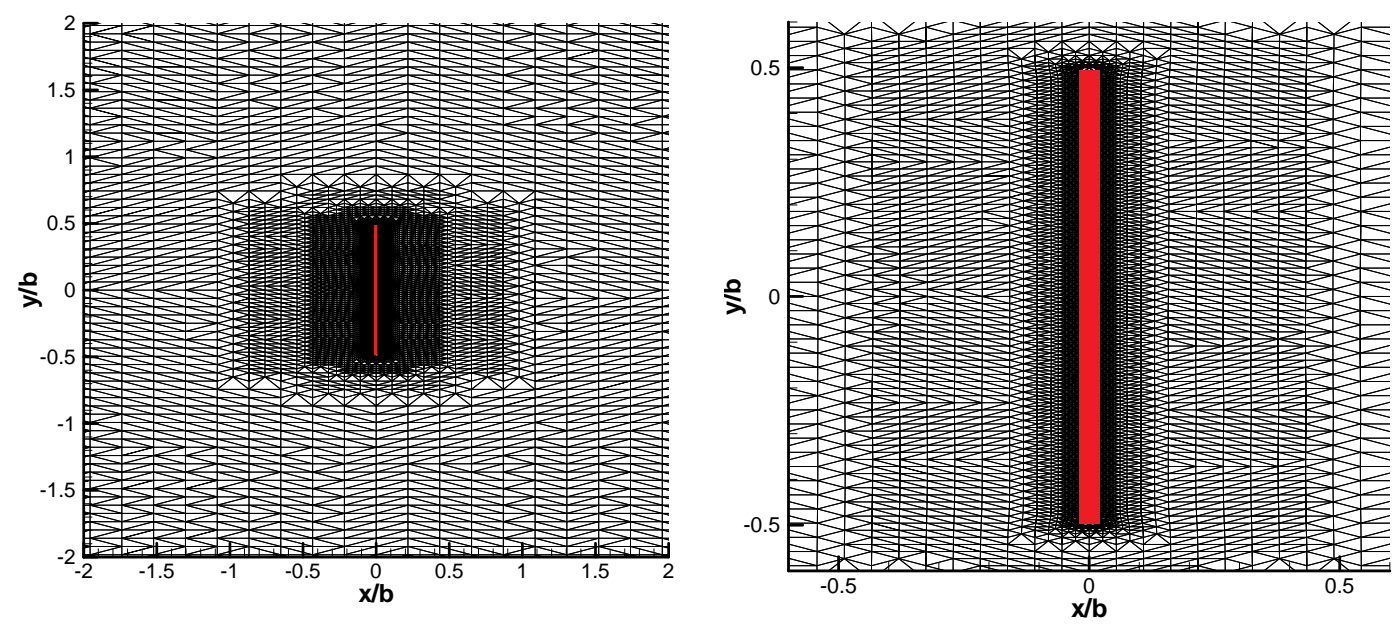

Figure 5.2: Scheme of the computational domain for the plane $z=0$

We use $<>_{t}$ to indicate time averaged quantities, \langle\rangle$_{s}$ for spanwise averaged values and $<>$ when quantities are spatio-temporally averaged. The $<>$ average is performed in two steps. Firstly, the time average is performed for each cell and finally an spatial average on the selected cells with the previously time averaged values. Both averages are computed as arithmetic mean values.

\subsubsection{Dimensionless parameters}

The plate is submerged in the lower phase fluid of density $\rho^{b}$ and viscosity $\mu^{b}$. The upper phase has a density $\rho^{t}<\rho^{b}$ and a viscosity $\mu^{t}$. Several non-dimensional numbers are used to study the problem, namely Reynolds number $(R e)$, Strouhal number $(S t)$, and the density ratio $\left(\rho^{b} / \rho^{t}\right)$ and viscosity ratio $\left(\mu^{b} / \mu^{t}\right)$. The first two are defined as:

$$
R e_{D_{h}}=\frac{\rho^{b} \bar{U} D_{h}}{\mu^{b}} \quad S t=\frac{f b}{\bar{U}}
$$

where $R e_{D_{h}}$ is based on the hydraulic diameter, defined as $D_{h}=\frac{2 \cdot b \cdot c}{b+c}$ when results are compared to those presented by Satheesh and Huera-Huarte (2019), or defined as $D_{h}=b$ when comparing results 
to those of Tian et al. (2014). In the Strouhal number, $f$ is the lift force frequency. Because the fluid properties are fixed, the non dimensional ratios $\rho^{b} / \rho^{t}$ and $\mu^{b} / \mu^{t}$ will not be changed throughout this work, and are fixed to 1000 and 55.24, respectively. The hydrodynamic forces acting on the streamwise and chordwise directions, $F_{x}$ and $F_{y}$ respectively, are non-dimensionalized in the form of drag $C_{D}$ and lift $C_{L}$ coefficients.

$$
C_{D}=\frac{F_{x}}{\frac{1}{2} \rho \bar{U}^{2} b c} \quad C_{L}=\frac{F_{y}}{\frac{1}{2} \rho \bar{U}^{2} b c}
$$

Finally, the boundary conditions are: mean velocity $\bar{U}$ for the inflow velocity and pressure is set as zero normal gradient. A no slip boundary condition for the velocity and a zero normal gradient for pressure are imposed on the plate. The top and bottom boundaries and the two planes in the spanwise direction are considered as walls, then no slip boundary condition for the velocity and a zero normal gradient for pressure are imposed. A zero-velocity gradient and zero-pressure condition is used for the outlet.

\subsection{Results: Validation of the numerical set-up}

Before analyzing the dynamics of the 3D flow around the submerged flat plate in cross-flow with the two phases involved, a single phase scenario is studied for validation. The validation case involves the flow around a 3D-periodic flat plate normal to the stream current. LES is performed at high Reynolds numbers $O\left(10^{5}\right)$, as in Tian et al. (2014). The drag coefficient, the characteristic flow frequencies and the pressure on the plate surface will be compared to the previous numerical results and with experiments found in the literature Fage and Johansen (1927). Other features, such as the variation of the vortex dynamics produced at the wake of the plate with the proximity to the free surface, are also described.

\begin{tabular}{|c|c|c|c|c|c|c|c|c|c|}
\hline & $b(\mathrm{~m})$ & $t / b$ & $K_{1} / b$ & $K / b$ & $c / b$ & $d / b$ & $G / b$ & $J / b$ & $\mathrm{AR}$ \\
\hline Tian et al. (2014) & 1 & 0.02 & 7.5 & 27.5 & 4 & - & 4 & 16 & 0.25 \\
\hline Satheesh and Huera-Huarte (2019) & $0.16,0.115$ & 0.0063 & 2.5 & 12.5 & 1,2 & 0 to 1.25 & 3.75 & 3.75 & $0.5,1$ \\
\hline
\end{tabular}

Table 5.1: Summary of the different parameters of the geometries proposed by Tian et al. (2014) and Satheesh and Huera-Huarte (2019) and the ones used in this work, see figure 5.1. For the case proposed in Satheesh and Huera-Huarte (2019), $G / b$, and $J / b$ are non-dimensionalized with the height of the plate $b=0.16 \mathrm{~m}$

The 3D-periodic case studied in Tian et al. (2014) with a single fluid is compared with the corresponding solutions obtained here. The characteristic length used in this problem is the height of the plate $b$. The dimensions of the global computational domain are consequently $K=27.5 b, J=16 b$ and 
$c=4 b$, identical to Tian et al. (2014). The flow inlet boundary is located $7.5 b$ upstream from the center of the plate, and the flow outlet is located $20 b$ downstream from the center of the plate. The plate has a flat rectangular cross-section with sharp corners and a thickness $t=0.02 b$. The Reynolds number based on $b$ is $R e=150000$, and the rest of the geometric values can be found in table 5.1. It is important to remark that in the geometry used in Tian et al. (2014) the corners are smooth with different curvatures of different radii, in our case, simulations are performed with sharp corners. As corners play a relevant role in these kind of flows, see Tian et al. (2014), some differences in the results are expected. A time step and mesh convergence process has been previously performed using several meshes and time steps in order to find global values such as the drag coefficient and the Strouhal number. For the sake of brevity, we are not including details of the whole extensive set of convergence tests performed, only the most representative results are referenced. The 3D computational domain has the same dimensions in the $x, y$ and $z$ directions and the sharp corners have been maintained.

A brief selection with some of the meshes used during the convergence process is presented in table 5.2. The finest grid is computed twice using two different sub-grid models, Smagorinsky and WALE, finding very little differences between them. The convergence trend in terms of drag coefficient is clear. The time step used in these simulations $\Delta t=5 \times 10^{-4}$ is very similar to the one used by Tian et al. (2014). Results show a good agreement with the experimental results described by Fage and Johansen (1927). The small differences between the results presented here, are comparable to those in found in the experiments and in previous computations, even though the geometry is not identical because of the round corners. It is evidenced here how meshes with less than 4.8 million cells (for 3D-periodic case proposed by Tian et al. (2014)) lead to large errors in the drag coefficient.

\begin{tabular}{|c|c|c|c|c|c|c|}
\hline Author & $<C_{D}>_{t}$ & Mesh Cells & cells in $x y$-Plane & cells in $z$ direction & Scheme & $\frac{\triangle t \bar{U}}{b}$ \\
\hline Fage and Johansen (1927) & 2.13 & - & - & - & Experiment & - \\
\hline Tian et al. (2014) & 2.20 & 9868800 & 102760 & 96 & Smagorinsky & $6 \times 10^{-4}$ \\
\hline Present work & 1.90 & 1450880 & 45500 & 32 & Smagorinsky & $5 \times 10^{-4}$ \\
\hline Present work & 2.42 & 4863600 & 108410 & 45 & Smagorinsky & $5 \times 10^{-4}$ \\
\hline Present work & 2.34 & 5709150 & 127260 & 45 & Smagorinsky & $5 \times 10^{-4}$ \\
\hline Present work & 2.20 & 5709150 & 127260 & 45 & WALE & $5 \times 10^{-4}$ \\
\hline Present work & 2.2408 & 5709150 & 127260 & 45 & Smagorinsky & $6 \times 10^{-4}$ \\
\hline Present work & 2.1429 & 5709150 & 127260 & 45 & Smagorinsky & $8 \times 10^{-4}$ \\
\hline
\end{tabular}

Table 5.2: 3D-periodic LES set-up validation. 
The spectrum of the resolved chordwise velocity fluctuations obtained at the mid-span point $x / b=1$, $y / b=0.5$ is shown in figure 5.3. As shown, the resolved scales appear to reach the inertial subrange, with a slope of approximately $-5 / 3$ Kravchenko et al. (2000), that indicates the turbulence spectrum has been correctly captured. In figure 5.3 , a clear peak $S t=0.159$ can be identified with the primary vortex shedding frequency, which is very close to the value 0.155 computed in Tian et al. (2014).

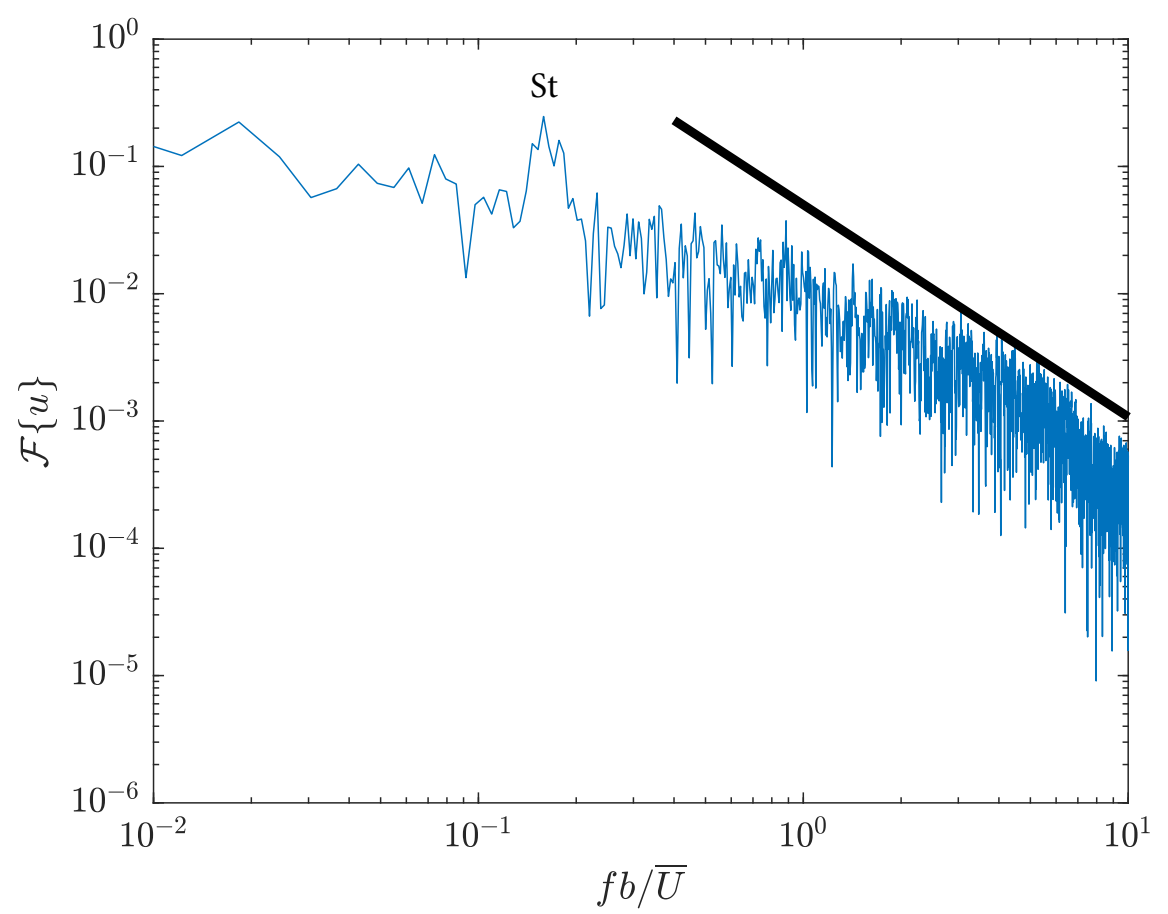

Figure 5.3: Spectrum of the resolved streamwise velocity fluctuations obtained at the point $x / b=1, y / b=0.5$ and $-5 / 3$ slope

The evolution of the drag $C_{D}$ and lift $C_{L}$ coefficients is plotted in figure 5.4. Similar to what was first presented by Najjar and Balachandar (1998), a high (H) and a low (L) drag regime, can be distinguished with the drag coefficient switching between them. The geometry of the plate corners has a very important role in the time evolution of the drag and lift coefficients, as shown by Tian et al. (2014). The authors reported how similar plates with different corners showed very different power spectra and characteristic frequencies. In our case, the absence of smooth corners, makes the problem more stiff from a numerical point of view. Despite this geometrical difference, as presented in figure 5.4, results are very similar to the ones in Tian et al. (2014). Particularly, the drag and lift ranges observed, $C_{D} \in[1.7,3]$ and $C_{L} \in[-0.32,0.3]$, and their corresponding time averaged values of 2.34 and $9.97 \times 10^{-6}$ for drag and lift respectively, compare very well with the magnitudes reported by Tian et al. (2014). Another important feature observed by Tian et al. (2014), and observed as well in the present results, is that the drag and lift fluctuations are closely related, with high drag intervals associated with large fluctuations of lift coefficient, as can be seen in figure 5.4. 

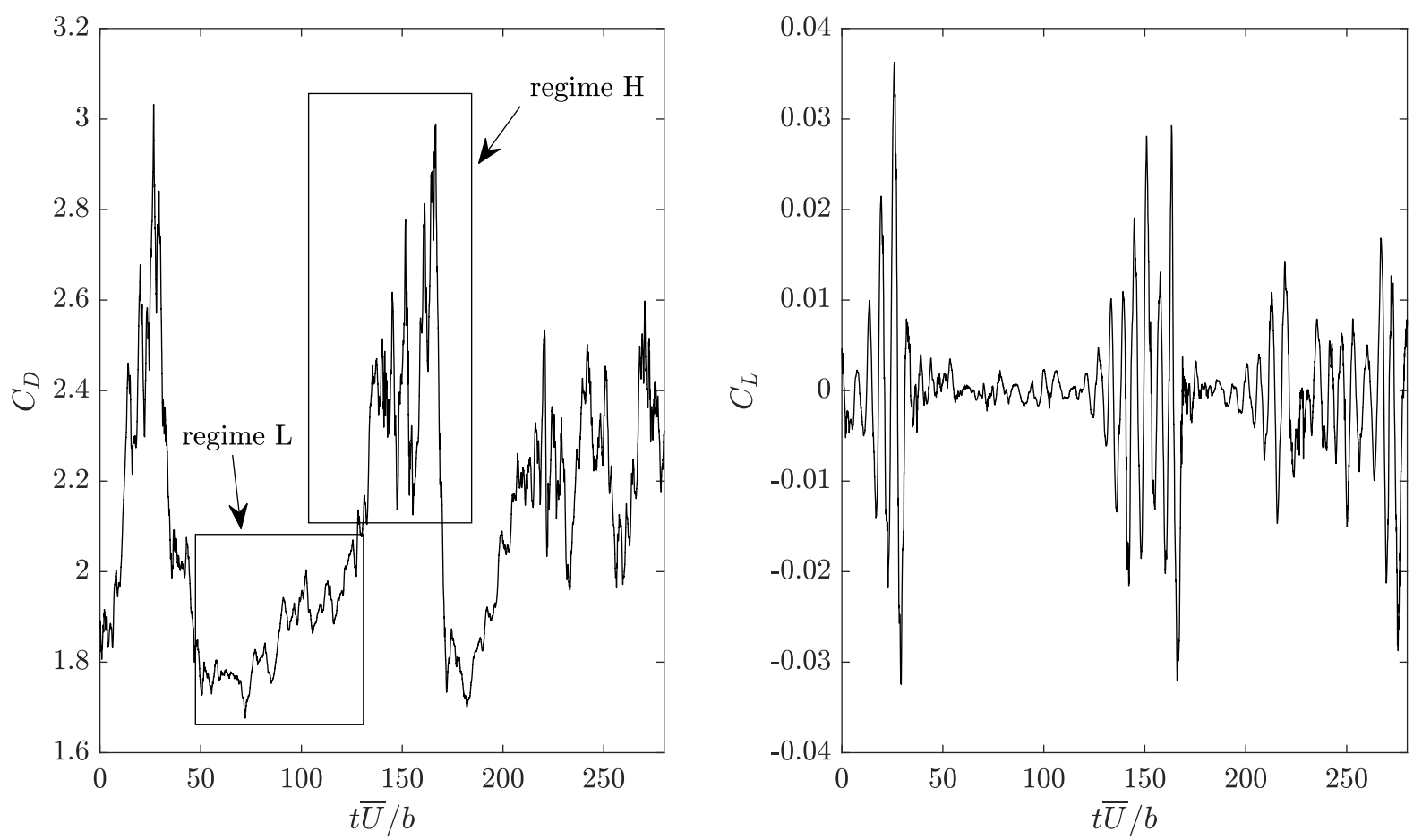

Figure 5.4: Evolution of the drag(left) and lift(right) coefficients for a single fluid in the 3D-periodic case.

The power spectrum of the drag $C_{D}$ and lift $C_{L}$ coefficients are plotted in figure 5.5. The low frequency unsteadiness that appears clear in the figures, was also observed by Tian et al. (2014), Najjar and Balachandar (1998) and Najjar and Vanka (1995), with the values of the amplitudes and the frequencies of the drag and lift spectra, being similar to the ones obtained at the same Reynolds number by Tian et al. (2014).

The distribution of the time and spanwise averaged streamwise velocity $\langle\bar{u}\rangle / \bar{U}$ along the wake center line $y=0$, is shown in figure 5.6. This curve shows the same trend as in the work by Tian et al. (2014), where the velocity changes its sign from negative to positive at the end of the recirculation bubble, $x / b \approx 2.4$. For larger values of $x$ the averaged streamwise component grows and finally stabilizes its value.

The time evolution of the three velocity components $(\bar{u}, \bar{v}, \bar{w})$ along a line in the spanwise direction is shown in figure 5.7. The line monitored is at $(x / b, y / b)=(1,0)$ near the center of the mean recirculation region. As observed in Tian et al. (2014), the low frequency unsteadiness that was observed in the power spectrum of the drag and lift coefficients, see figure 5.5, is also clearly observed here in the cross-stream middle plot. It also happens, that the $\bar{v}$ component exhibits a larger amplitude if compared to the other two components. Another interesting phenomenon is the synchronization of the large drag values with the cross-stream velocity fluctuation. The $\mathrm{L}$ and $\mathrm{H}$ regimes can also be identified if figures 

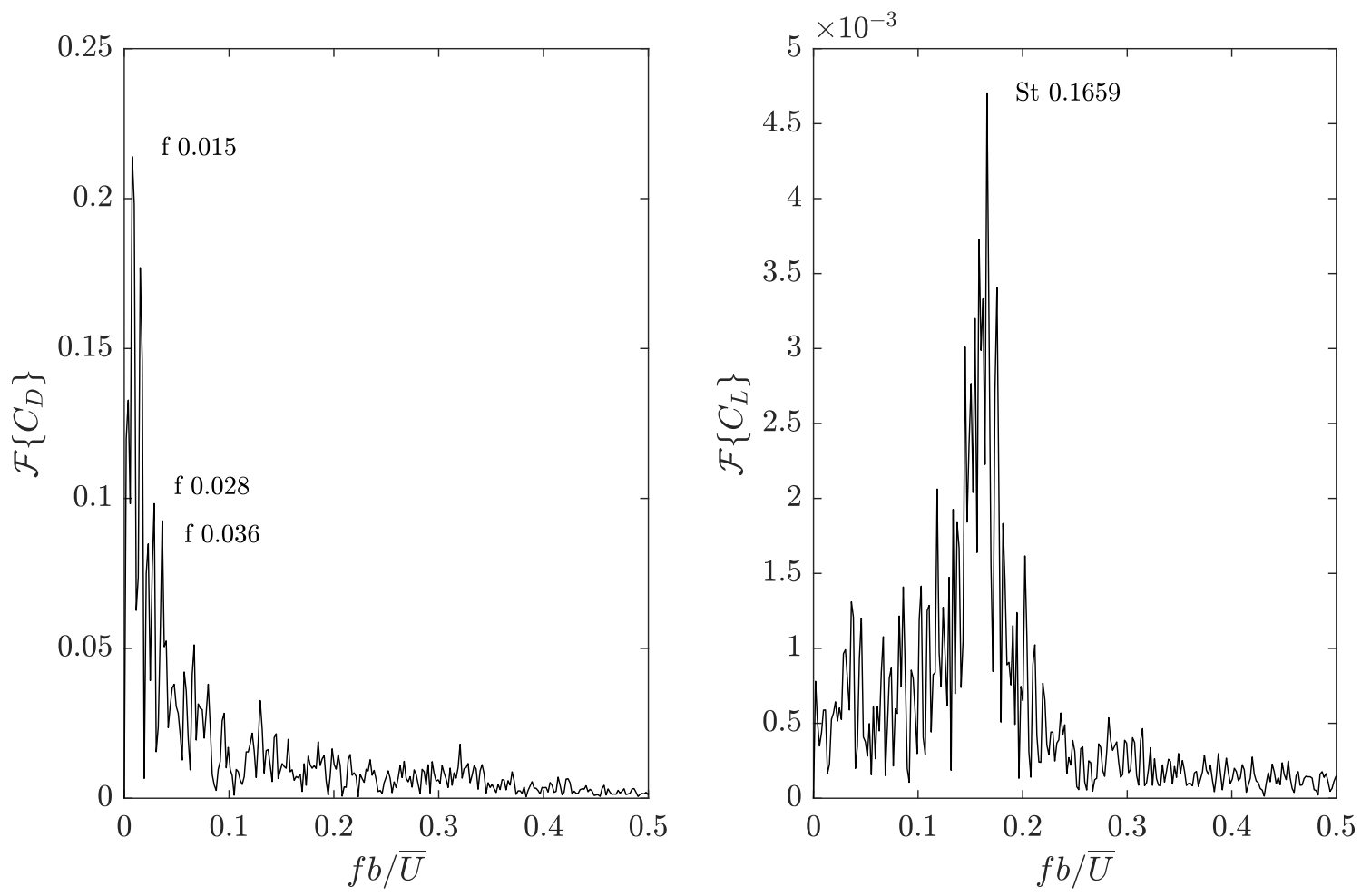

Figure 5.5: Power spectra of the time evolution of the drag(left) and lift(right) coefficients for the single fluid 3Dperiodic case.

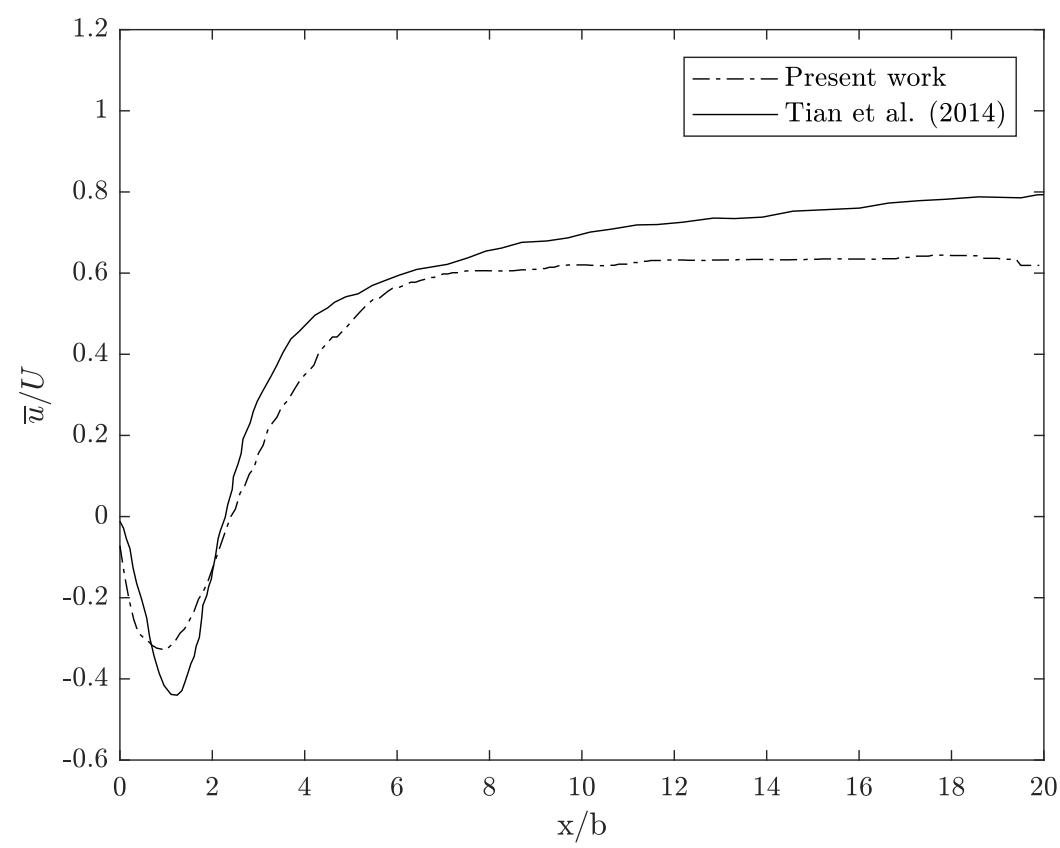

Figure 5.6: Time and spanwise averaged streamwise velocity $u$ along the wake centerline $y=0$. 
5.5(right) and 5.7(top) are compared.
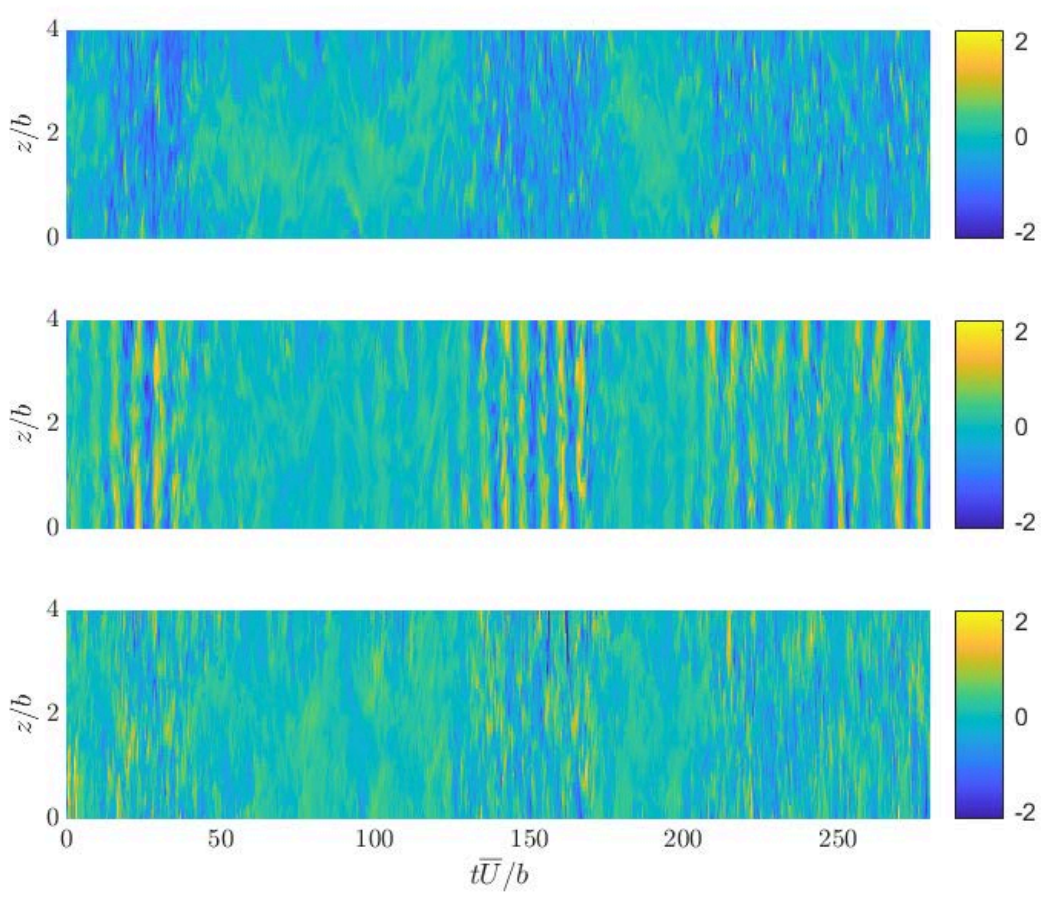

Figure 5.7: Time evolution of velocities: $\bar{u}($ top $), \bar{v}$ (middle), $\bar{w}$ (bottom) along the line $x / b=1, y / b=0$ for the 3Dperiodic case.

Figure 5.8 shows the mean pressure coefficient $<C_{p}>$ distribution, with $C_{p}=\frac{p-p_{\infty}}{\frac{1}{2} \rho U^{2}}$, on the upstream and the downstream sides of the rigid plate. The results by Tian et al. (2014) obtained with plates with smooth corners are also plotted for comparison. As it can be observed, the $\left\langle C_{p}>\right.$ distributions are very similar. For the present work, the pressure is just represented on both sides of the plate and not on the lateral surfaces, as a consequence a jump appears at $y / D= \pm 0.5$. As we explained before, the small differences found in the pressure distribution could be justified by the geometrical differences due to the presence of round corners in the work by Tian et al. (2014).

\subsection{Results: Full 3D simulations involving free surface}

In this section, 3D LES numerical simulations are conducted on the rectangular flat plate in cross-flow near the free surface. The work presented here is inspired by the experiments described in Satheesh and Huera-Huarte (2019), where a rectangular flat plate was towed in a water tank with a cross-section of $0.6 \times 0.6 \mathrm{~m}^{2}$. In our computational domain, dimensions match those in the experiments as indicated in figure 5.1 .

The aspect ratio of the plate is defined as $A R=b / c$ where $b$ is the vertical dimension and $c$ is the horizontal dimension (parallel to the free surface). Two aspect ratios are simulated here, in-between 


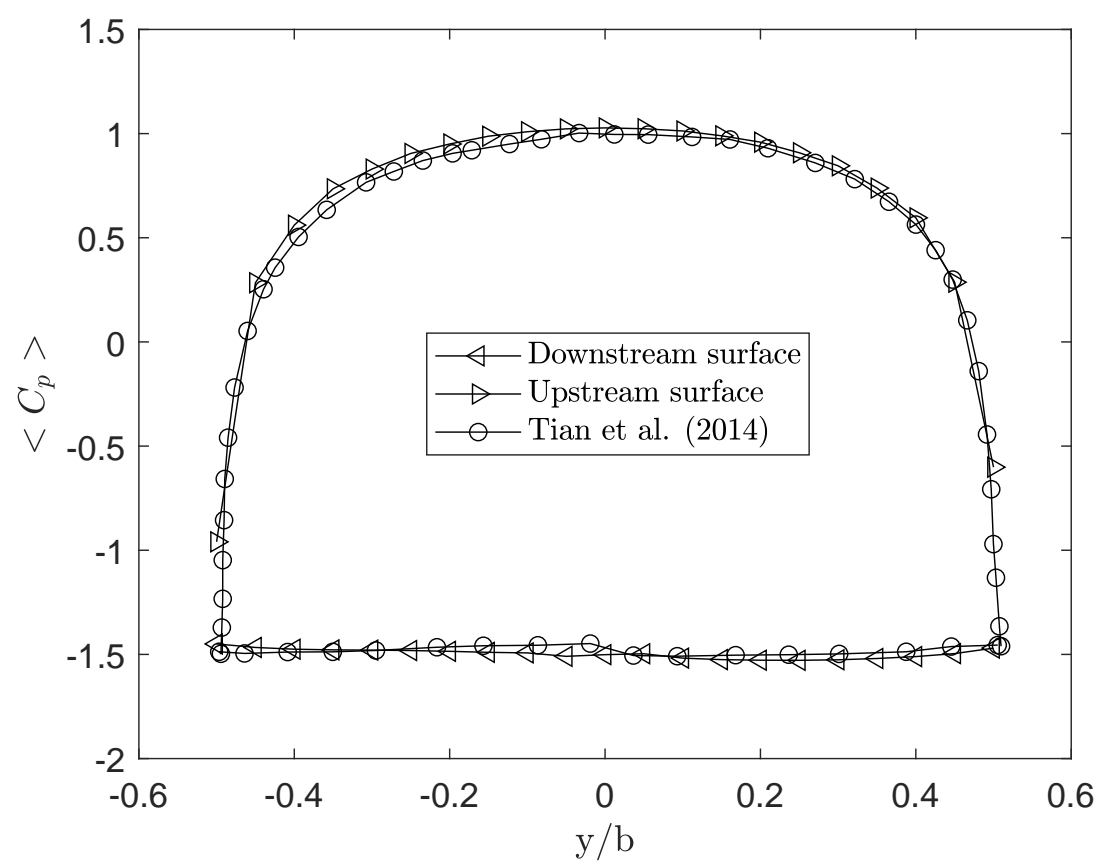

Figure 5.8: Time and spanwise mean pressure coefficient $\left\langle C_{p}>\right.$ distribution on the front and back sides of the plate for the 3D-periodic case.

those appearing in the experiments by Satheesh and Huera-Huarte (2019). The first is 0.5 ( $b=0.115$ $\mathrm{m}$ and $c=0.23 \mathrm{~m})$ and the second is $1(b, c=0.16 \mathrm{~m})$, chosen because they showed a very different drag coefficient trend in the experiments. All plates have the same thickness $t=5 \times 10^{-3} \mathrm{~m}$, as detailed in figure 5.1. Three inflow velocities have been investigated, with values $0.2,0.3$ and $0.4 \mathrm{~m} / \mathrm{s}$, leading to Reynolds numbers of $30 \times 10^{3}, 45 \times 10^{3}$ and $60 \times 10^{3}$, if based on the hydraulic diameter $\left(D_{h}=\frac{2 \cdot b \cdot c}{b+c}\right)$. Following the experiment design by Satheesh and Huera-Huarte (2019), for each $A R$, the same submergence depths for the plates have been computed. Depths $(d)$ are measured from the upper edge of the plate to the undisturbed free surface. For the $A R=0.5$ the depths in dimensionless form $(d / b)$ are $0,0.0870,0.2174,0.4348,0.6087,0.7826,1.2609$ and 1.9130 , whilst for the case with the plate of $A R=1$, the depths are $0,0.0625,0.1250,0.1875,0.3125,0.6250$ and 1.25 . Note that in the experimental work by Satheesh and Huera-Huarte (2019), the chord $c$ was used non-dimensionalisation and here, the span $b$ is used as in the previous section.

Different meshes have been constructed following the validation exercise presented in section A.3. The number of cells varies from $3.5 \times 10^{6}$ to $6 \times 10^{6}$ depending on the plate aspect ratio $A R$. In order to better understand the effect of the free surface, a case in which the free surface is replaced by a horizontal solid wall has been also included. For this case the distance $d / b$ represents the distance from the top of the plate to the solid wall.

The results obtained for the plate of $A R=1$ appear in figure 5.9, where a comparison between the experimental and the computational mean drag coefficient values is presented for different Reynolds 
numbers and submergence depths. The data suggests that the drag coefficient dependency on Reynolds number is very small. Moreover, both the experimental and the computational results present a saturation behaviour when the submergence depth is large, implying a very limited influence of the free surface, as expected. In general, mean drag increases monotonically as the submergence depth is reduced from the domain centre, up to a position at which drag is maximum, to start decreasing again until the upper edge of the plate is at the free surface. The numerical simulations show that the location of the maxima in drag is $R e$ dependent, but always takes place at $d / b<0.3$. The experimental data reported in Satheesh and Huera-Huarte (2019) showed how in general, for all the aspect ratios and Re investigated, the peak in the drag coefficient took place as well at $d / b<0.3$, well in agreement with the numerical results presented here. A very similar drag trend is observed if the aspect ratio is reduced to $A R=0.5$, see figure 5.10. Again, as the plate is moved away of the free surface, drag increases up to a maximum to start decreasing to the stable values found at the centre of the domain. In order to further understand the role of free surface, a series of numerical cases were computed after removing of the problem the gravity force and changing the free surface by a rigid wall. This was only done for the plate with $A R=1$ at a $R e=60000$, as it can be seen the lower plot of figure 5.9. Under these circumstances, $d / b$ is the dimensionless distance from the top part of the plate to the fixed upper wall. The results show a similar trend in the drag coefficient if compared to the free surface case, although the highest drag value takes place at a smaller submergence depth $(d / b \approx 0.0625)$. The discrepancies found in figures 5.9 and 5.10 could be justified by the presence of the experimental setup necessary to hold and tow the plate along the channel which has a relevant influence on the measurements, of course those elements are not considered in the numerical approach. We should also remark that no error bars are reported in the experimental results.

The analysis of the pressure distribution around the plate can help in elucidating the origin of the drag coefficient trends observed. In figure 5.11, spatio-temporal averaged dimensionless pressure coefficient $<C_{p}>$ difference between the upstream and the downstream sides of the $A R=1$ plate along $y / b$, appears for the different depths, at a $R e=60000$. As can be observed, in figure 5.11 , the $\left\langle C_{p}>\right.$ variation is linear along the vertical direction $y / b$ which clearly corresponds to an hydrostatic dominated pressure distribution. As the hydrostatic component of the pressure acts symmetrically at both sides of the plate, it does not contribute to the drag value. The drag coefficient is dominated by the pressure force component, with the ratio between the pressure and the friction drag components in order $O\left(10^{5}\right)$ Tian et al. (2014). The pressure difference between the front and the back sides of the plate, $\left.\Delta<C_{p}\right\rangle$ can be associated to the pressure contribution to the drag value, and in order to visualize the influence of the depth on it, $\Delta<C_{p}>$ appears plotted against $y / b$ in figure 5.11. Larger the values of $d / b$ are, the more symmetric $\Delta<C_{p}>$ distribution is, meaning that the free surface influence is negligible at large depths. On the other side, for low values of $d / b$, the influence of the free surface is represented by a 

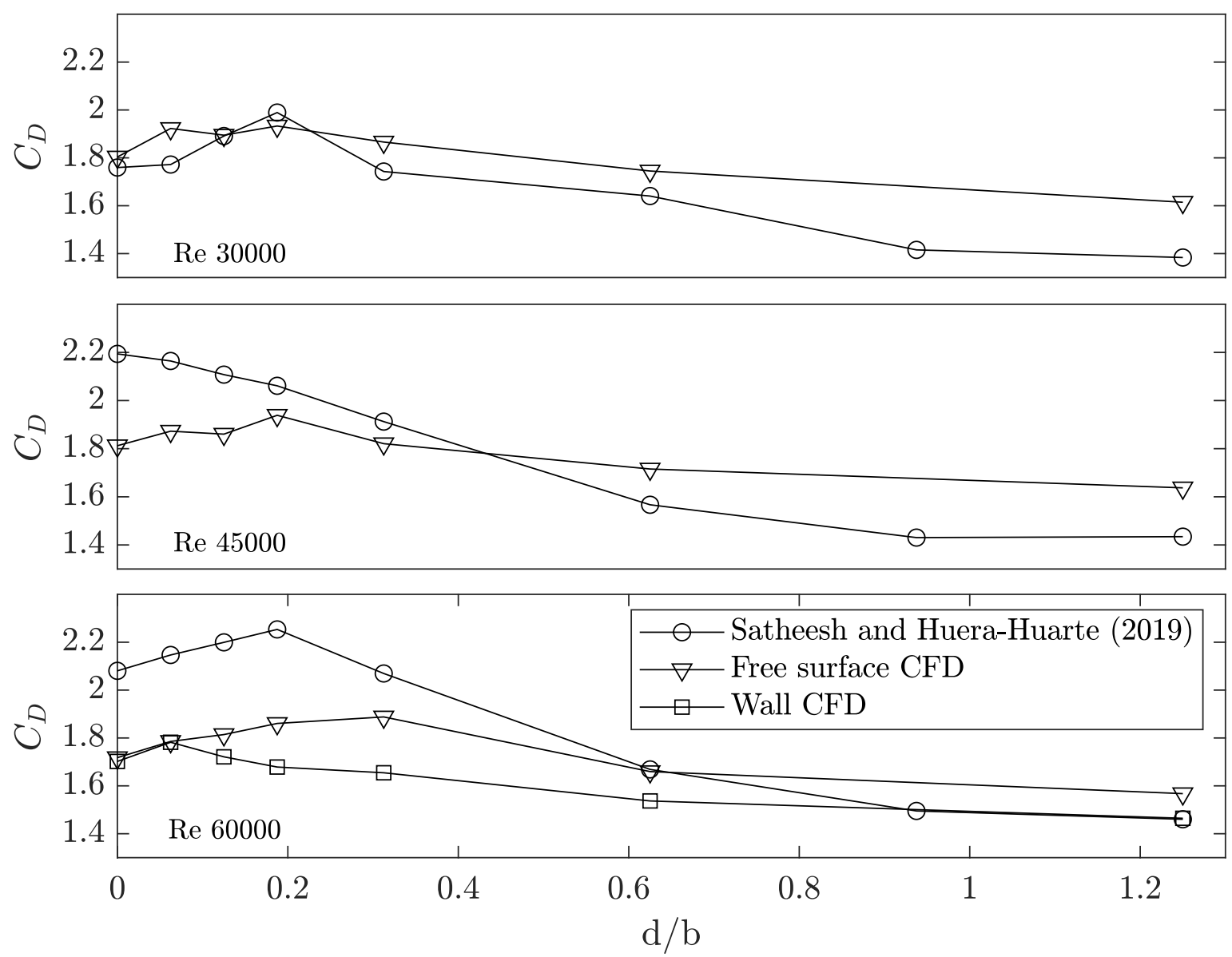

Figure 5.9: Averaged drag coefficients $C_{D}$ for different depths when the plate aspect ratio $A R=1$ and $R e=$ $30 \times 10^{3}$ (top), $R e=45 \times 10^{3}$ (middle), $R e=60 \times 10^{3}$ (bottom). For $R e=60 \times 10^{3}$ the results for the complementary problem using a top wall instead of free surface have been added for comparison.

clear asymmetry in the curve, with a clear increment on the part of the plate closer to the free surface (positive range of the $y / b$ ). Another important fact is the variation of the quantity $\int_{-b / 2}^{b / 2} \Delta<C_{p}>d y$ for different immersion depths, which represents the pressure component of the drag coefficient. As it can be observed in figure 5.12, the maximum takes place at $d / b=0.3125$, which is in good agreement with the results previously shown in the lower plot of figure 5.9. The case of the plate near the solid wall is also shown in the plots for reference. The first difference is that due to the absence of the hydrostatic component the $\left\langle C_{p}>\right.$, values only contain the dynamic part and the difference between the front and back sides of the plate, is more evident. The values of $\Delta<C_{p}>$ and $\int_{-b / 2}^{b / 2} \Delta<C_{p}>d y$ are also represented. In contrast with the free surface case, the maximum value of the integral term is found at $d / b=0.0625$, with a maximum value almost equal to the case with the free surface. The trend of the curve in figure 5.13 matches well with that presented in the lower plot of figure 5.9. 

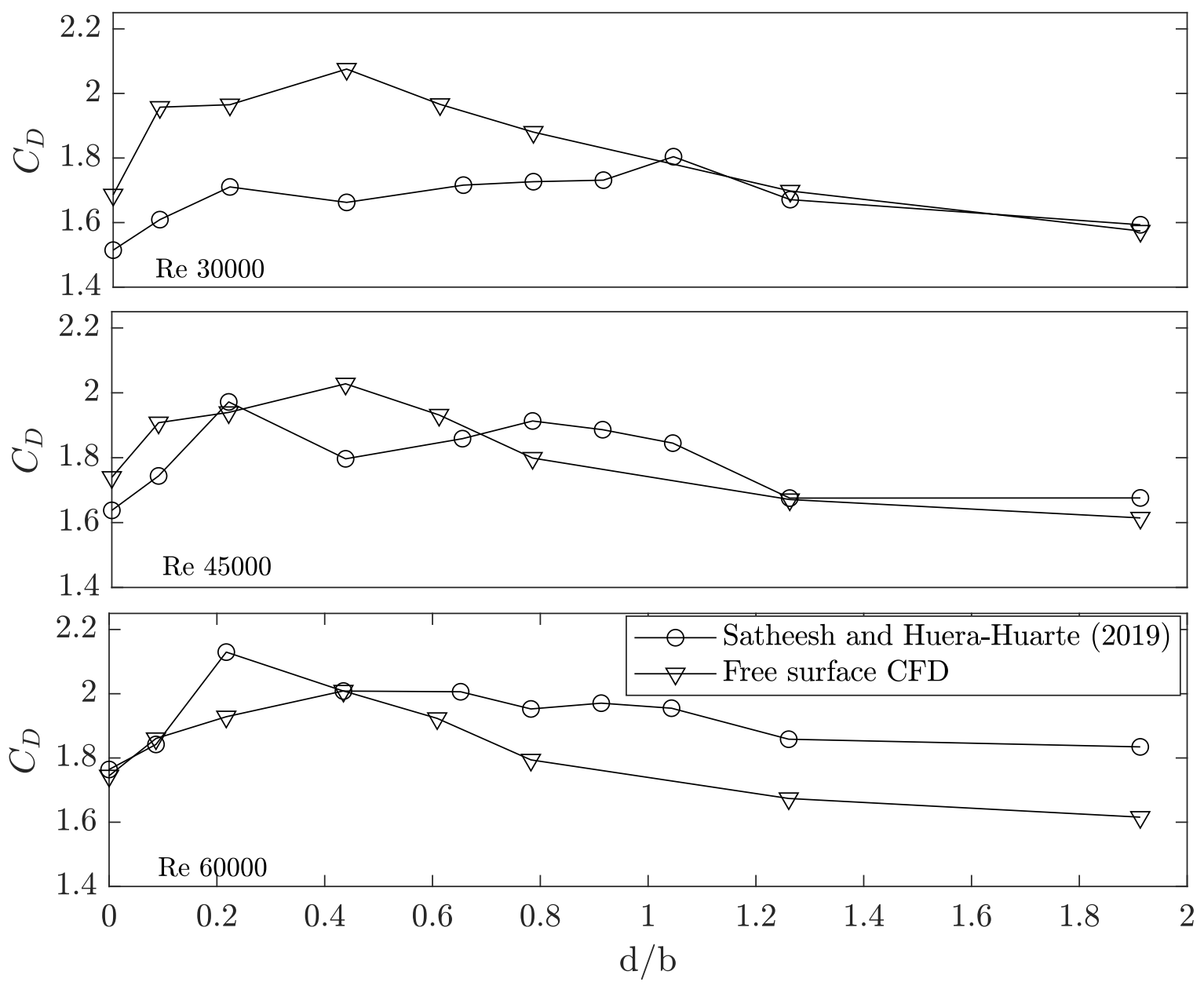

Figure 5.10: Averaged drag coefficient $C_{D}$ against depth $d / b$ when the plate aspect ratio $A R=0.5$ and $R e=$ $30 \times 10^{3}$ (top), $R e=45 \times 10^{3}$ (middle), $R e=60 \times 10^{3}$ (bottom)

Regarding frequencies, the spectra of the resolved cross-stream velocity fluctuations obtained at the mid-span for $x / b=1$ and $z / b=0$, for three different depths with dimensionless value of $0,0.125$ and 0.625 , are shown in figure 5.14. According to the turbulence spectrum presented, the present simulations capture the inherent turbulent cascade where the resolved scales reach the inertial subrange described by the $-5 / 3$ Kolmogorov slope.

In figure 5.15 the evolution of the drag and lift forces are presented for the case with $A R=1$ and $R e=60000$, at the largest depth investigated of $d / b=1.25$, either with free surface or with a solid wall. The drag values are $C_{D}=1.57 \pm 2.5 \%$ for the free surface case, and $C_{D}=1.43 \pm 2.7 \%$ for the wall case. Fluctuations are very small if compared to their mean value. This result is completely different to the one shown for the periodic validation case in section $\mathrm{A} .3$ or those that appear in any of the $2 \mathrm{D}$ or 3D-periodic cases available in the literature, see for example the work by Hemmati et al. (2016a) where with $R e$ of either 1200 or 2400 , two regimes $\mathrm{H}$ and $\mathrm{L}$ can be distinguished. In the present $3 \mathrm{D}$ simulations, 


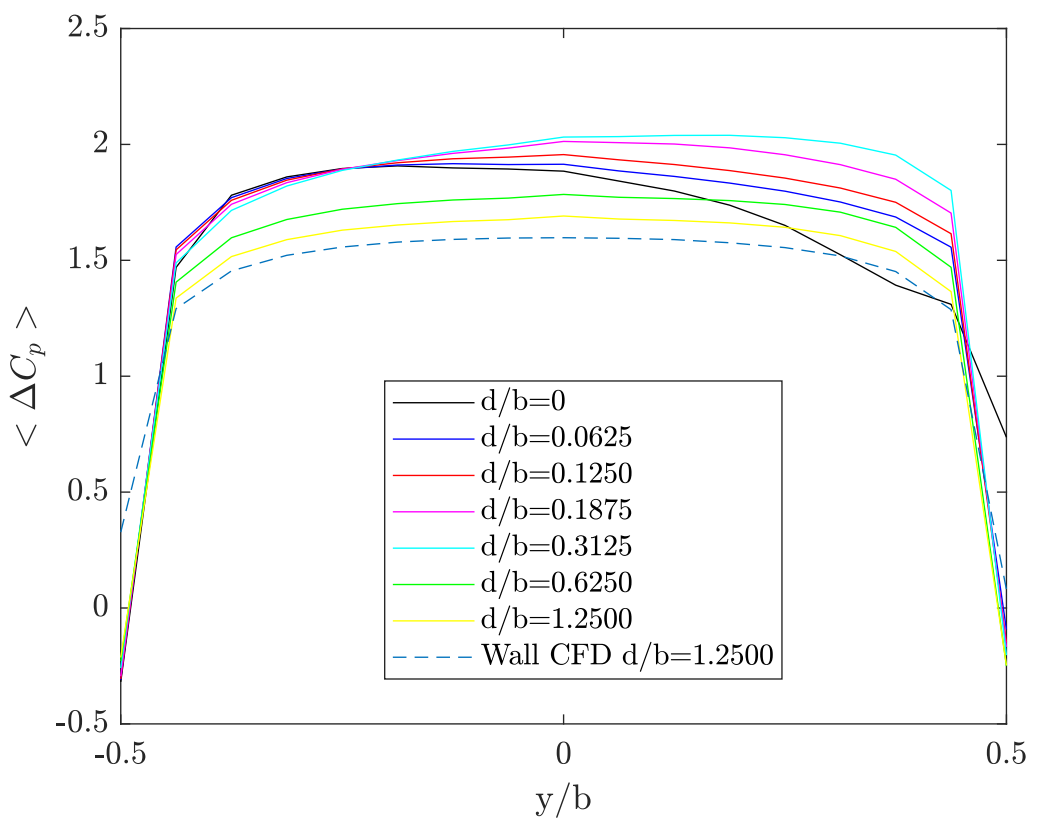

Figure 5.11: Time and cross averaged dimensionless pressure coefficient $\left\langle C_{p}\right\rangle$ difference between front and back sides along $y / b$ for $A R=1$ and $R e=60 k$ for different depths $d / b$.

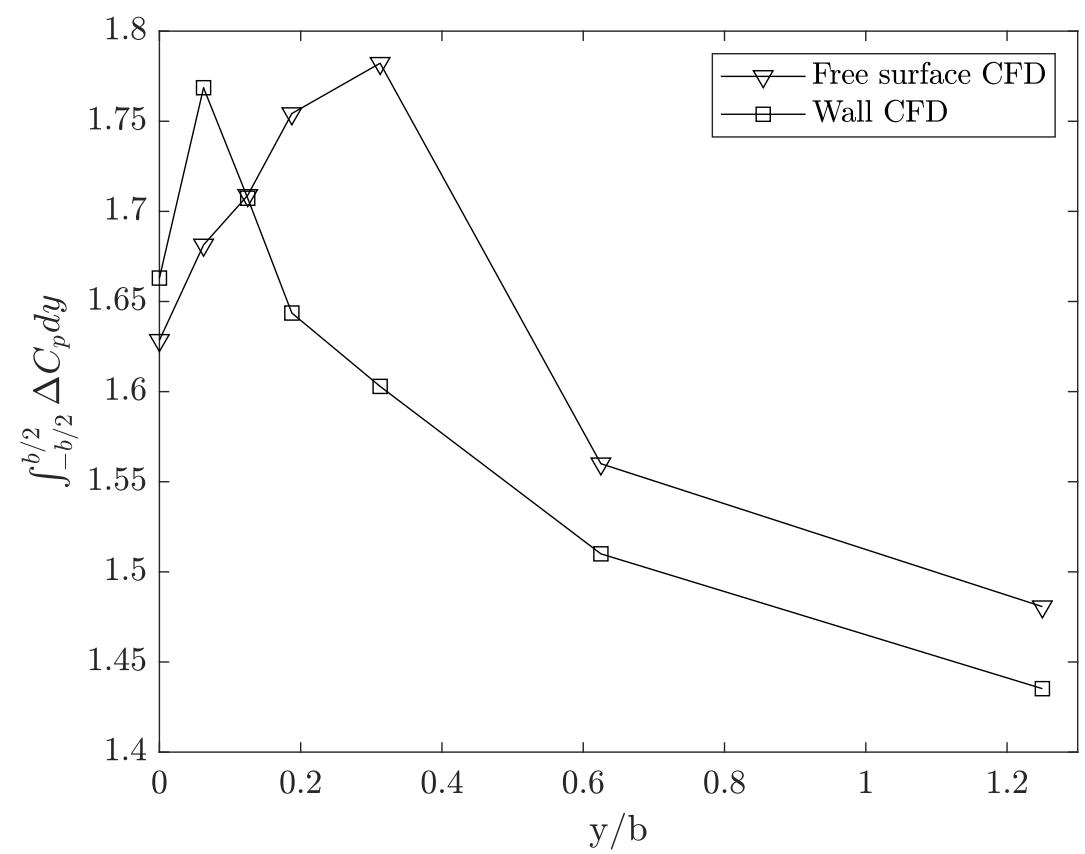

Figure 5.12: Integral value of the pressure coefficient difference between both sides of the plate along the vertical direction. 

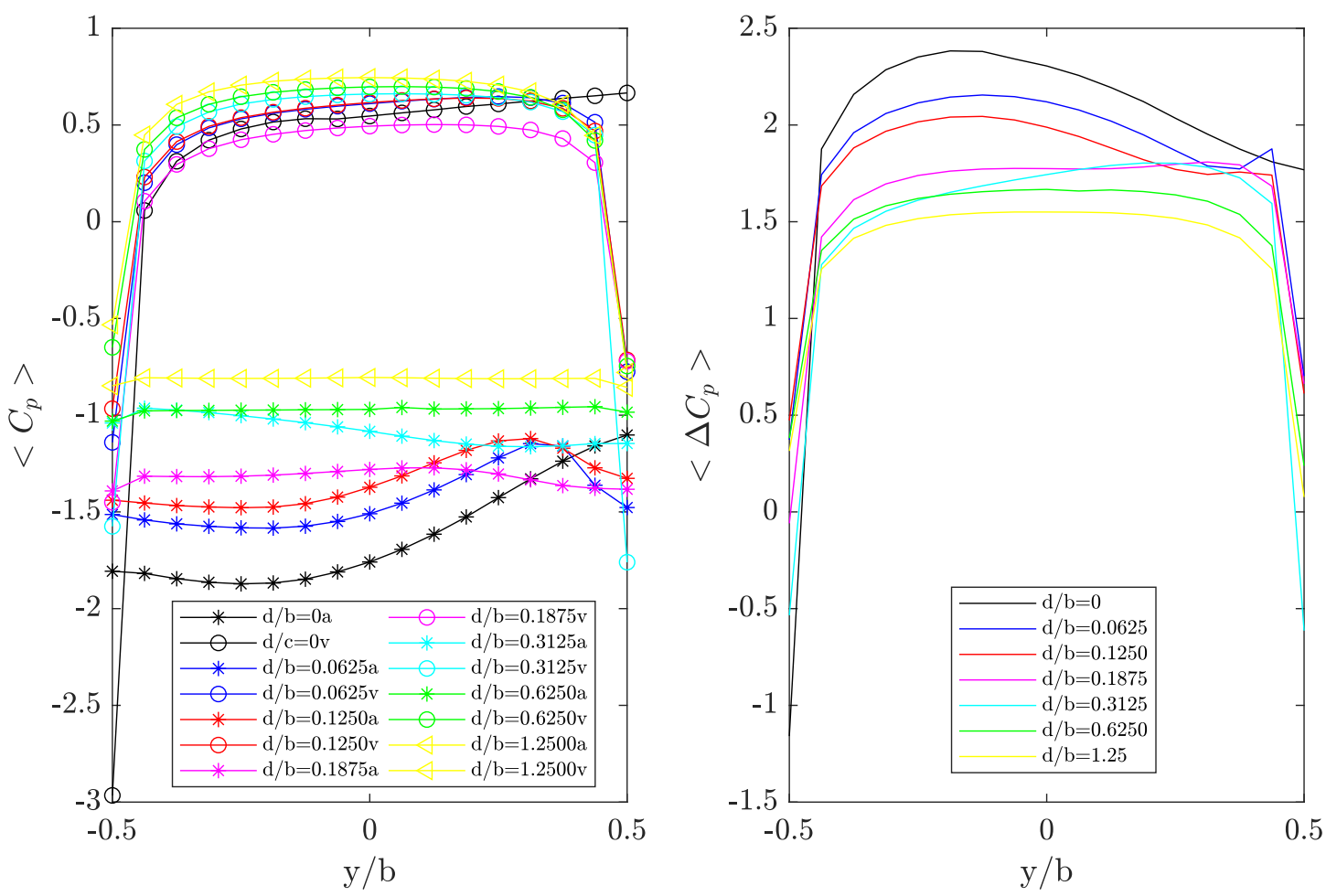

Figure 5.13: Time and cross averaged dimensionless pressure coefficient $\left\langle C_{p}>\right.$ along $y / c$ for $A R=1$ and $R e=60 k$ for different wall distances $d / b$. Circles represent the front side, $-v$ letter when $d / b$ case is indicated, while asterisks represent the back side, $a$ letter when $d / b$ case is indicated. Right: Time and cross averaged dimensionless pressure coefficient $\left\langle C_{p}\right\rangle$ difference between front and back sides along $y / b$ for $A R=1$ and $R e=60 k$ for different depths $d / b$.

with no periodic assumption between the lateral walls, and conducted at higher $R e$, those regimes do not appear as the drag oscillates with a very small amplitude close to its mean value. The same applies to the case computed with the plate with a top rigid boundary, also presented in figure 5.15, therefore it seems obvious that it is not the presence of the free surface that is causing the lack of oscillations of the forces and the disappearance of the $\mathrm{H}$ and $\mathrm{L}$ regimes. In the case with the rigid wall the drag and lift fluctuations are again very small. As expected, the amplitudes represented in the spectral analysis of the drag and lift signals, see figure 5.16, are orders of magnitude smaller than those obtained in the validation case of section A.3, see figure 5.5. This low amplitude spectrum does not allow to consider any characteristic frequencies to be compared to the crosswise periodic case, neither for the drag nor for the lift forces. This scenario with drag and lift forces that barely oscillate around their mean value, is consistent at all depths studied.

Following the detailed analysis of the forcing terms and the comparisons made with previous experiments and numerical work, the wake dynamics is studied, with the objective to further discuss the physics of the free surface interaction with the plate. Figures 5.17 and 5.18 depict the time averaged 


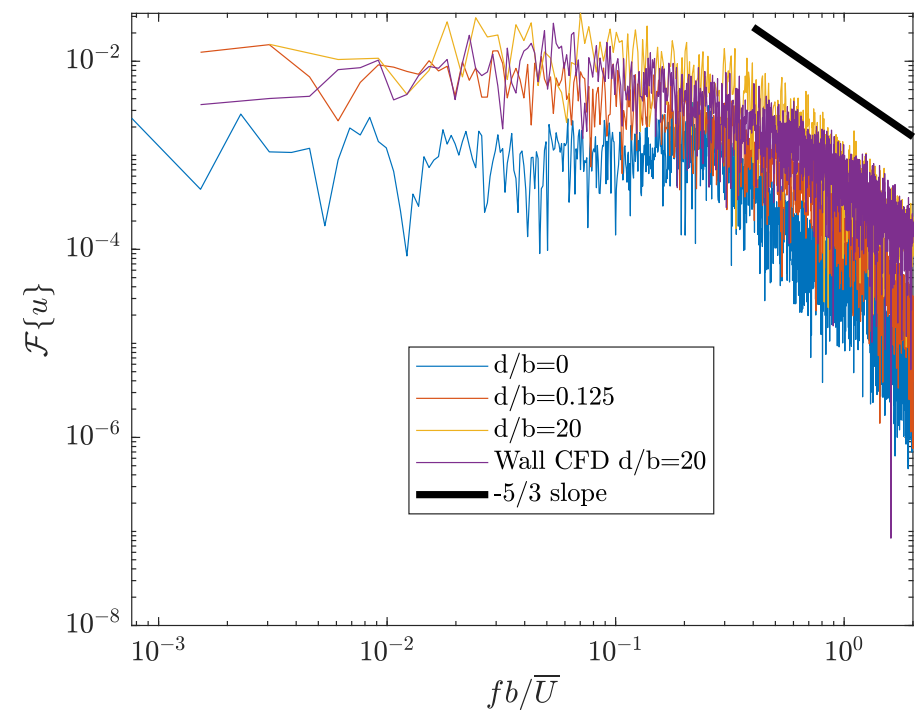

Figure 5.14: Spectra of the resolved cross-stream velocity fluctuations obtained at the mid - span $x / b=1$ and $z / b=0$ for three different depths; $d / b=0, d / b=0.125$ and $d / b=0.625$ when $A R=1$ and $R e=60 k$.
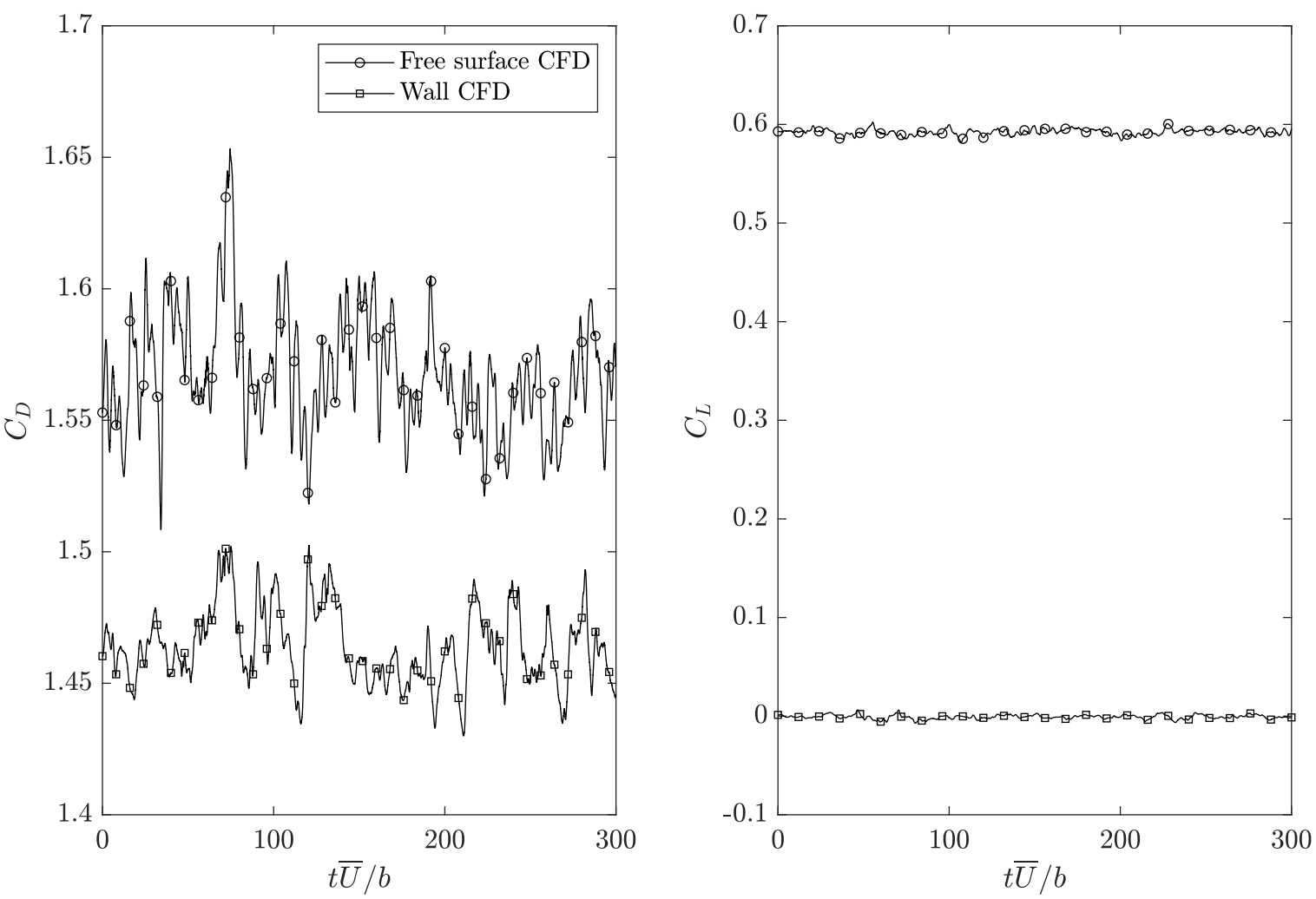

Figure 5.15: Evolution of the $\operatorname{drag}($ left) and lift(right) coefficients when $A R=1, R e=60 k$ and $d / b=1.25$ 

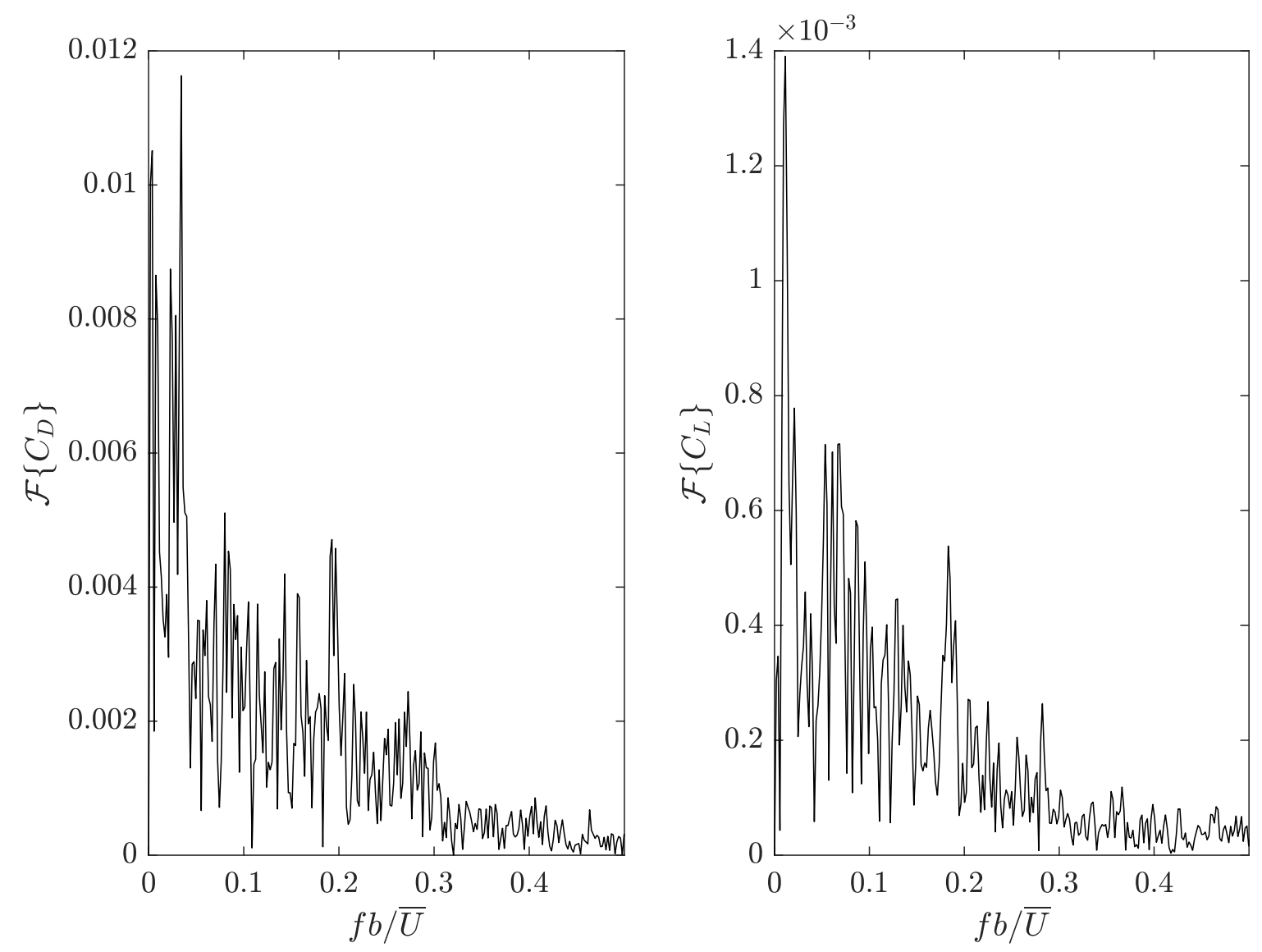

Figure 5.16: Power spectra of the time evolution of the drag(left) and lift(right) coefficients when $A R=1, R e=60 k$ and $d / b=1.25$

streamline distribution for the case with the free surface and the case of the solid wall, respectively. In both figures the Reynolds number $R e_{D_{h}}=60000$ and the aspect ratio is $A R=1$. The dimensionless time window used for averaging process is $t U / b \in[0-300]$, with data that was saved every $\Delta(t U / b)=0.1$.

When the distance to the upper boundary is maximum $(d / b=1.25)$, the streamlines on the wake show two symmetric counter-rotating vortices with a streamwise recirculation length $L r_{x} \sim 2.5 b$. The flow at the maximum depth is practically the same for the case with free surface (figure 5.17) and the one with the rigid wall (figure 5.18), with the main difference being the upward bending of the streamlines in the far wake for the case with free surface, as a result of the pressure distribution created by the gravity field, which is non existing in the rigid wall case. Very similar results were obtained by Hemmati et al. (2016a) and Taneda and Honji (1971) for laminar flows and by Tian et al. (2014) for turbulent flows. The wake vortex dynamics change considerably when the plate approaches either the free surface or the solid wall. The counter-rotating pair tends to deform, breaking the previously observed symmetry. In the case involving the free surface, both vortices remain in the wake at all depths, but their relative positions 
are heavily dependent on the depth. Secondary vortices appear at the upper part of the plate, due to the free surface deformation, when depths are $0.3125,0.187$ and 0.1125 . At these specific depths, the main upper vortex and the secondary one form a counter-rotating pair near the free surface that creates a jetlike flow (or gap flow) accelerating the flow between the upper edge of the plate and the boundary. The free surface is able to deform and to create a small depression behind the plate that modifies the flow characteristics at the top part of the plate and allows some streamlines to overpass the plate following the free surface distortion.

With a top solid wall boundary this complex dynamics between the counter-rotating vortices disappears at depth $d / b=0.3125$, where the upper side vortex vanishes and only the lower one dominates. If the depth is further reduced the upper vortex appears again. Finally, when the plate contacts the wall, this top vortex is barely observed and a large counter-clockwise recirculation bubble dominates the wake. As the top boundary cannot be deformed, the flow through the gap is limited and the tends to the formation of an stagnation point as the gap decreases. In the limit case $d / b=0$, no flow circulates between the top part of the plate and the solid wall.

In figure 5.19, initial formation and steady state snapshots of the computed dimensionless spanwise stage of the vortex $\omega_{z}$ are compared to the experimental results shown by Satheesh and Huera-Huarte (2019) for depths $d / b$ of $0,0.217,0.434$ and 1.913 for the plate of $A R=0.5$ and $R e_{D_{h}}=45000$. The dimensionless size of the PIV domain reported by Satheesh and Huera-Huarte (2019) was limited to $(y / b, z / b) \in[-1,1] x[0,2.5]$ because of the measurement set-up, whilst the computational domain shown here is larger $(x / b, y / b) \in[-1.8,1.8] \times[-0.5,4]$, as the same limitations do not apply to the computations. Experiments and numerical simulations show the same features. Vorticity tends to have a symmetric distribution as depth is increased, see $d / b=1.913$ in figure 5.19 as an example. As the plate is moved closer to the free surface, the vortex distribution looses symmetry. For the cases $d / b=0.434$ and $d / b=0.217$ in figure 5.19, the vorticity created near the free surface modifies the evolution of the upper shear layer. The generation of vorticity at the deformed free surface creates the jet-like flow described before, leaving a very weak upper shear layer, and a flow channelled towards the lower part of the plate. The same phenomena takes place again at a depth $d / b=0.434$. When the plate is located at the free surface, with a $d / b=0$, the injection of fluid through the gap flow becomes so small that no vorticity is generated in the upper part of the plate. The modification of the free surface is small as well and the secondary vortex disappears. The size of the region that the shear layer encloses when the upper edge of plate is at the free surface, is considerably bigger than in the case with $d / b=1.913$. The enlargement of this region moves away from the plate the low pressure region, resulting in a drag reduction.

An analysis of the pressure field explains why the low $(\mathrm{L})$ and high $(\mathrm{H})$ drag regimes described by Tian et al. (2014) and Hemmati et al. (2016a), at $R e=150000$ and $R e=1200$ respectively, are missing in the full 3D simulations presented here. In figure 5.20, an example of instantaneous dimensionless 


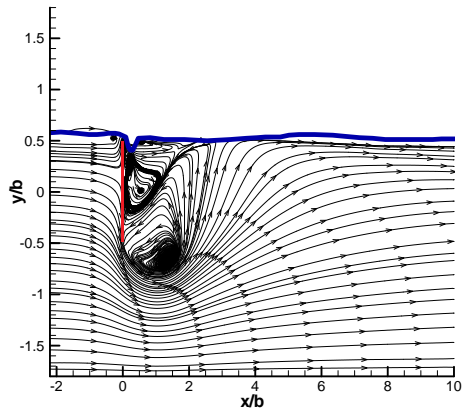

(a) $d / b=0$

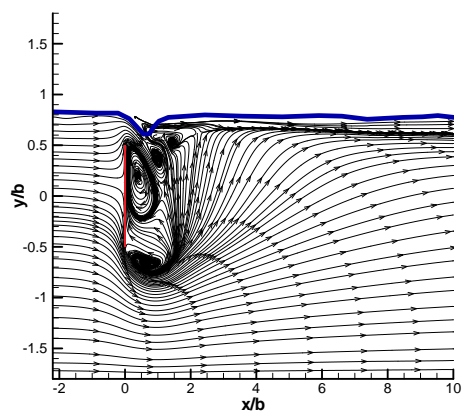

(d) $d / b=0.187$

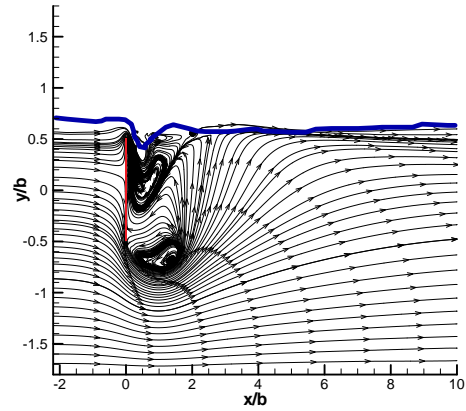

(b) $d / b=0.0625$

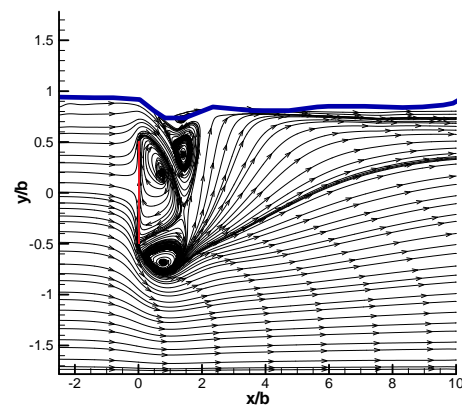

(e) $d / b=0.3125$

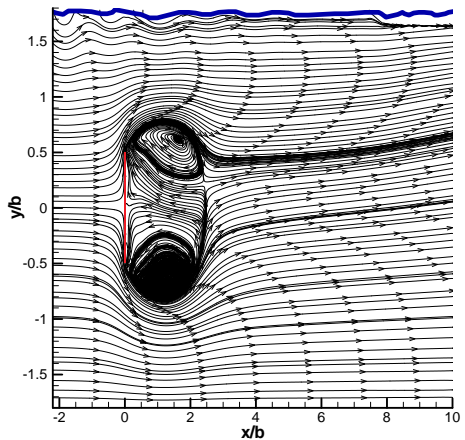

(g) $d / b=1.25$

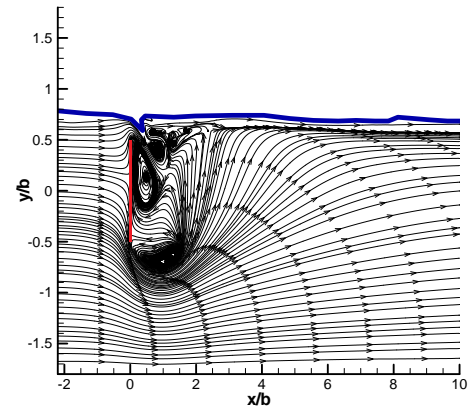

(c) $d / b=0.1125$

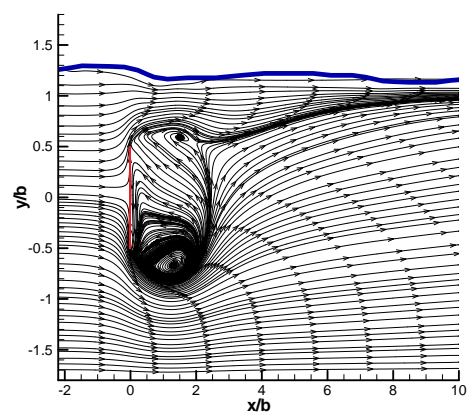

(f) $d / b=0.625$

Figure 5.17: Streamlines of the time and spanwise averaged flow field in the near wake at different depths $d / b$.

pressure field $\left(p^{*}=\frac{p}{\rho \bar{U}^{2}}\right)$ for the case of the plate with $A R=1, R e_{D_{h}}=60000$ and $d / b=1.25$, is shown. In the lower plot, instantaneous streamlines are presented for the same case. Two high and low pressure regions are found at the bow and the aft of the plate respectively, the stability of these regions causes the low drag coefficient variation previously reported. These regions can be seen in figure 5.20, in the form of two counter-rotating vortices at $x / b \sim 1$ in the streamline plot.

Finally, the Q criterion Jeong and Hussain (1995) has been used to present the vortex structures in the turbulent wake. In particular, the differences in the flow between the case with the solid wall and its free surface counterpart, are highlighted. Figures 5.21 and 5.22 show the instantaneous iso-surface of vorticity $(Q=1.0)$, for cases with depth $d / b=1.25$ and $R e=60000$. Generally, the wake structures that appear in figure 5.21 are much more complex than those resulting from the 3D-periodic cases 


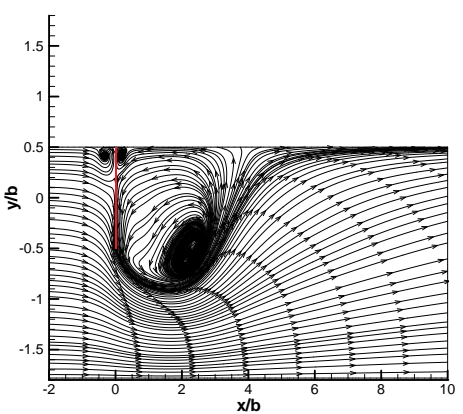

(a) $d / b=0$

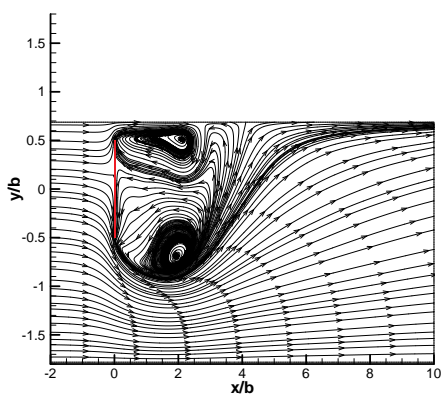

(d) $d / b=0.1875$

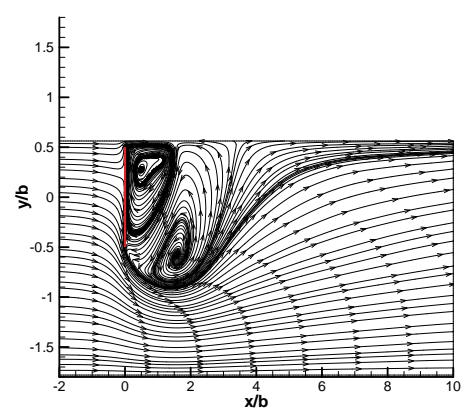

(b) $d / b=0.0625$

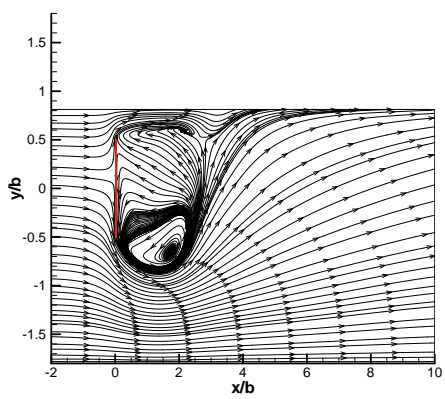

(e) $d / b=0.3125$

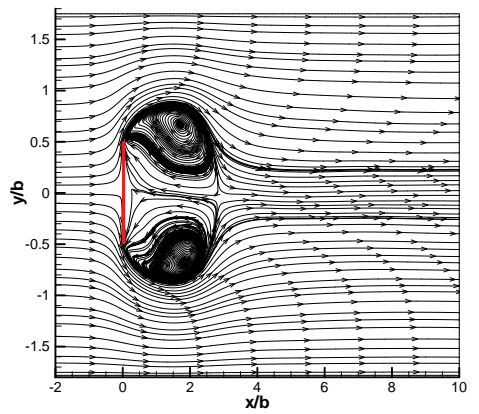

(g) $d / b=1.25$

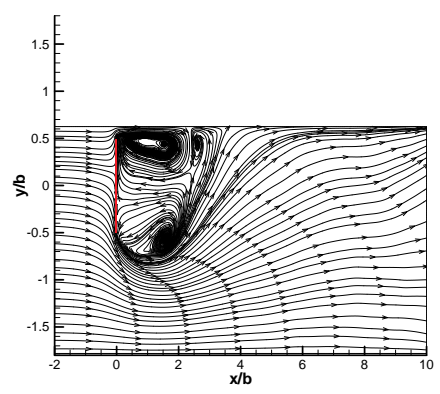

(c) $d / b=0.125$

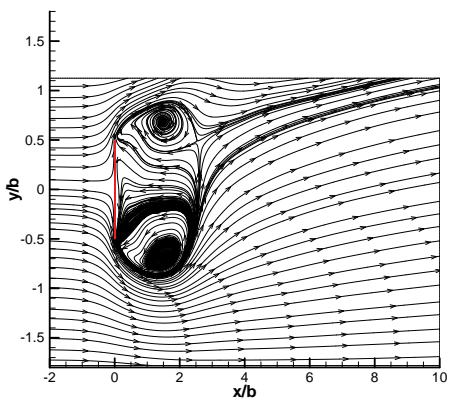

(f) $d / b=0.625$

Figure 5.18: Streamlines of the time and spanwise averaged flow field in the near wake at different wall distances $d / b$

presented by Hemmati et al. (2016b), for lower Reynolds numbers in the order of 1200. A separation distance between consecutive vortex loops cannot be well identified, showing the typical complexity of a turbulent wake. The $x z$ perspective in figure 5.21 presents an alternating global structure similar to the 3D-periodic case presented by Tian et al. (2014), where the width of the vortex street increases as the flow moves downstream, this global trend is not so evident when the free surface is considered. Vortex structures for the free surface case are stretched in the streamwise direction, whilst in the case with the rigid wall, in figure 5.21, smaller vortex structures are formed in the near wake at $x / b \sim 1$. The dynamics described by Hemmati et al. (2016b) and Hemmati et al. (2016a) appear as well here, with the alternate shedding of large scale structures at each side of the wake. The peel off process is also observed, with a streamwise flow induced away from the plate by the main vortices, that carries out the smaller vortices 